$$
\text { for }
$$

\title{
Palladium/Copper-Cocatalyzed Arylsilylation of Internal Alkynes with Acyl Fluorides and Silylboranes: Synthesis of Tetrasubstituted Alkenylsilanes by Three-Component Coupling Reaction
}

\author{
Qiang Chen, ${ }^{a}$ Zhenyao Li, ${ }^{a}$ and Yasushi Nishihara ${ }^{* b}$ \\ ${ }^{a}$ Graduate School of Natural Science and Technology, Okayama University, \\ 3-1-1 Tsushimanaka, Kita-ku, Okayama 700-8530, Japan \\ ${ }^{b}$ Research Institute for Interdisciplinary Science, Okayama University, \\ 3-1-1 Tsushimanaka, Kita-ku, Okayama 700-8530, Japan \\ Phone: $+81-86-251-7855$ \\ Fax: +81-86-251-7855 \\ Email: ynishiha@okayama-u.ac.jp
}

\section{Table of Contents}

1. General

2. Synthesis of IPrCuOTf

3.Optimization of Reaction Conditions

4. Experimental Procedures and Spectroscopic Data for the Products

$S 12-S 28$

5. Mechanistic Studies

S29-S30

6. Copies of ${ }^{1} H,{ }^{13} C\left\{{ }^{1} H\right\}$, and ${ }^{19} F\left\{{ }^{1} H\right\}$ NMR Charts for the Products

S31-S66

7. References 


\section{General}

\section{Instrumentation}

Unless otherwise noted, all the reactions were carried out under Ar atmosphere using standard Schlenk techniques. Solvents were employed as eluents for all other routine operation, as well as dehydrated solvent were purchased from commercial suppliers and employed without any further purification. Glassware was dried in an oven $\left(130^{\circ} \mathrm{C}\right)$ and heated under reduced pressure before use. For thin layer chromatography (TLC) analyses throughout this work, Merck precoated TLC plates (silica gel $60 \mathrm{GF}_{254}, 0.25 \mathrm{~mm}$ ) were used. Silica gel column chromatography was carried out using Silica gel $60 \mathrm{~N}$ (spherical, neutral, 40-100 $\mu \mathrm{m}$ ) from Kanto Chemicals Co., Ltd. NMR spectra $\left({ }^{1} \mathrm{H},{ }^{13} \mathrm{C}\left\{{ }^{1} \mathrm{H}\right\}\right.$, and $\left.{ }^{19} \mathrm{~F}\left\{{ }^{1} \mathrm{H}\right\}\right)$ were recorded on Mercury-400 (400 MHz) spectrometers. Chemical shifts $(\delta)$ are in parts per million relative to $\mathrm{CDCl}_{3}$ at $7.26 \mathrm{ppm}$ for ${ }^{1} \mathrm{H}$ and at 77.16 ppm for ${ }^{13} \mathrm{C}\left\{{ }^{1} \mathrm{H}\right\}$, respectively. The ${ }^{19} \mathrm{~F}\left\{{ }^{1} \mathrm{H}\right\}$ NMR spectra were measured by using $\mathrm{CCl}_{3} \mathrm{~F}(\delta=0.00 \mathrm{ppm})$ as an external standard. The GC yields were determined by GC analysis of the crude mixture, using $n$-dodecane as an internal standard. GC analyses were performed on a Shimadzu GC-14A equipped with a flame ionization detector using Shimadzu Capillary Column (CBP1-M25-025) and Shimadzu C-R6A-Chromatopac integrator. Infrared spectra were recorded on a Shimadzu IR Prestige-21 spectrophotometer. Elemental analyses were carried out with a Perkin-Elmer 2400 CHN elemental analyzer at Okayama University.

\section{Chemicals}

Unless otherwise noted, materials obtained from commercial suppliers were used without further purification. Palladium(II)chloride and toluene, 1,4-dioxane (super dehydrated) were purchased from Wako Chemical Co. 4,5-Bis(diphenylphosphino)-9,9-dimethylxanthene, bis[(2-diphenylphosphino)phenyl] ether, 1-phenyl-1propyne, 1-phenyl-1-butyne and benzoyl fluoride (1a) (purity > 98\%) were purchased from TCI. Diphenylacetylene was purchased from Sigma-Aldrich. Acyl fluorides $\mathbf{1 b}-\mathbf{1} \mathbf{s}^{1}$, silylborane $\mathbf{2}^{2}$ and internal alkynes $(\mathbf{3 a - 3 e}, \mathbf{3 h})^{\mathbf{3}}$ were prepared according to the literatures. 


\section{Synthesis of IPrCuOTf}

\section{$N, N^{\prime}-1,4-B i s(2,6-d i i s o p r o p y l p h e n y l)-1,4-d i a z a b u t a d i e n e^{4}$}

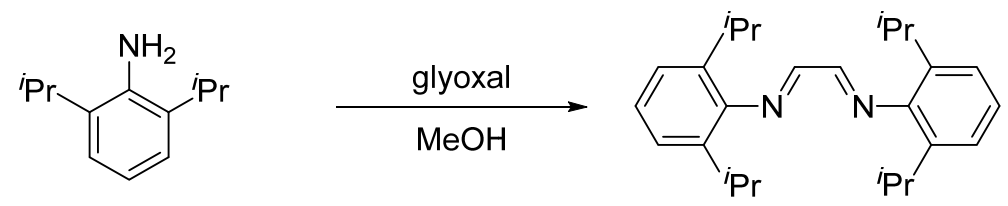

To a solution of 2,6-diisopropylaniline $(8.86 \mathrm{~g}, 50 \mathrm{mmol})$ in $\mathrm{MeOH}(50 \mathrm{~mL})$ was added a solution of glyoxal ( $40 \%$ in water) $(3.6 \mathrm{~mL}, 25 \mathrm{mmol})$ in air. The mixture was stirred at room temperature overnight. The reaction mixture was filtered, then the filter cake was washed with $\mathrm{MeOH}(3 \times 20 \mathrm{~mL})$ and dried in vacuo to afford 6.78 $\mathrm{g}$ of the title compound as a yellow solid (72\% yield). ${ }^{1} \mathrm{H}$ NMR (400 $\left.\mathrm{MHz}, \mathrm{CDCl}_{3}\right): \delta 8.12(\mathrm{~s}, 2 \mathrm{H}), 7.22-7.15$ (m, 6H), 2.99-2.92 (m, 4H), $1.22(\mathrm{~d}, J=8.0 \mathrm{~Hz}, 24 \mathrm{H})$.

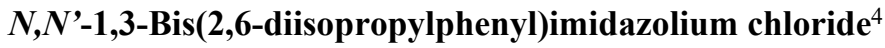
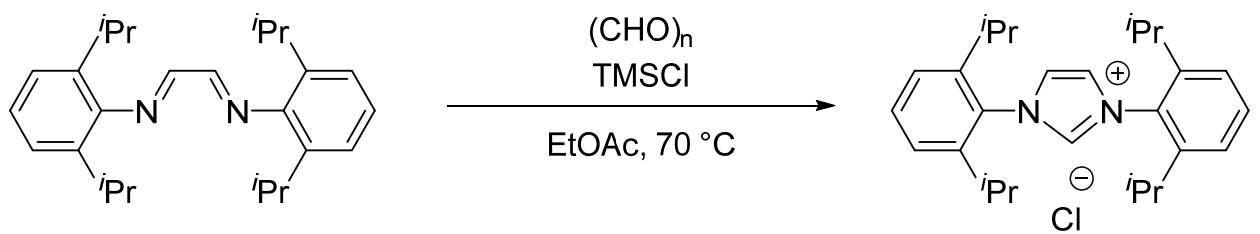

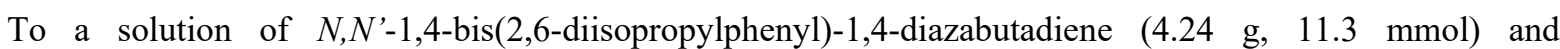
paraformaldehyde $(0.35 \mathrm{~g}, 11.6 \mathrm{mmol})$ in EtOAc $(100 \mathrm{~L})$ was added a solution of trimethylsilyl chloride $(1.5 \mathrm{~mL}$, $11.6 \mathrm{mmol})$ in EtOAc $(30 \mathrm{~mL})$ at $70{ }^{\circ} \mathrm{C}$ dropwise over $45 \mathrm{~min}$ in air. The mixture was stirred at $70{ }^{\circ} \mathrm{C}$ for $2 \mathrm{~h}$, then cooled to $10^{\circ} \mathrm{C}$. The reaction mixture was filtered, then the filter cake was washed with EtOAc $(3 \times 10$ $\mathrm{mL})$ and dried in vacuo to afford $3.36 \mathrm{~g}$ (70\% yield) of the title compound as a white solid. ${ }^{1} \mathrm{H} \mathrm{NMR}(400 \mathrm{MHz}$, $\left.\mathrm{CDCl}_{3}\right): \delta 10.02(\mathrm{~d}, J=2.0 \mathrm{~Hz}, 1 \mathrm{H}), 8.14(\mathrm{~d}, J=1.6 \mathrm{~Hz}, 2 \mathrm{H}), 7.56(\mathrm{t}, J=7.8 \mathrm{~Hz}, 2 \mathrm{H}), 7.34(\mathrm{~d}, J=8.0 \mathrm{~Hz}, 4 \mathrm{H})$, 2.47-2.40 (m, 4H), 1.29-1.22 (m, 24H).

\section{[1,3-Bis(2,6-di-iso-propylphenyl)imidazol-2-ylidene] copper (I) chloride, (IPr)CuCl ${ }^{5}$}<smiles></smiles><smiles>CC(C)(Cl)O[Na]</smiles><smiles>CC(C)c1cccc(C(C)C)c1N1C=CN(c2c(C(C)C)cccc2C(C)C)C1Cl</smiles> 
An oven-dried Schlenk flask containing 1,3-bis(2,6-di-isopropylphenyl) imidazolium chloride (849.0 mg, 2.0 $\mathrm{mmol}), \mathrm{CuCl}$ (198 mg, $2.0 \mathrm{mmol}), \mathrm{NaOt}-\mathrm{Bu}(192 \mathrm{mg}, 2.0 \mathrm{mmol})$ was evacuated and refilled with argon three times. THF $(10 \mathrm{~mL})$ were added and the resulting suspension was stirred at room temperature for $4 \mathrm{~h}$. Then, the reaction mixture was filtered through Celite to give the title compound as a white powder $(788.6 \mathrm{mg}, 81 \%$ yield). ${ }^{1} \mathrm{H}$ NMR (400 MHz, $\left.\mathrm{CDCl}_{3}\right): \delta 7.49(\mathrm{t}, J=8.0 \mathrm{~Hz}, 2 \mathrm{H}), 7.30(\mathrm{~d}, J=7.6 \mathrm{~Hz}, 4 \mathrm{H}), 7.13(\mathrm{~s}, 2 \mathrm{H}), 2.60-2.53$ $(\mathrm{m}, 4 \mathrm{H}), 1.31-1.22(\mathrm{~m}, 24 \mathrm{H})$.

\section{[1,3-Bis(2,6-di-iso-propylphenyl)imidazol-2-ylidene] copper (I) triflate, (IPr)Cu(OTf) ${ }^{6}$}
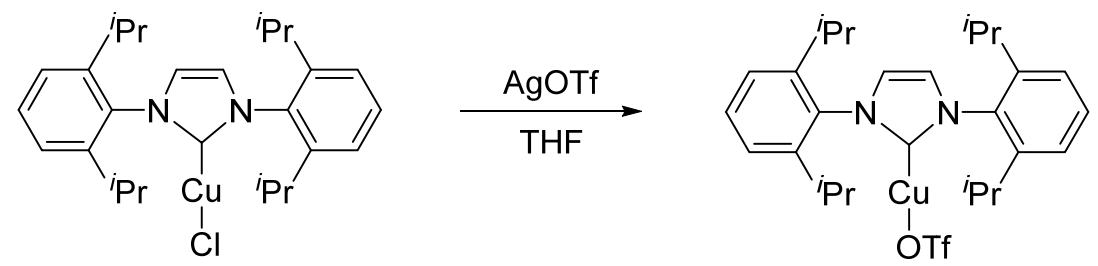

$(\operatorname{IPr}) \mathrm{CuCl}(0.251 \mathrm{~g}, 0.52 \mathrm{mmol})$ was combined with $\operatorname{AgOTf}(0.133 \mathrm{~g}, 0.52 \mathrm{mmol})$ in $25 \mathrm{~mL}$ of THF. The solution turned brown after $5 \mathrm{~min}$ with stirring. After stirring for $30 \mathrm{~min}, \mathrm{AgCl}$ was removed by filtration through Celite, and the filtrate was concentrated to approximately $5 \mathrm{~mL}$ under vacuum. Hexane $(10 \mathrm{~mL}) \mathrm{was}$ added to form white powder $(0.234 \mathrm{~g}, 77 \%$ yield), which was collected via filtration and dried under reduced pressure. ${ }^{1} \mathrm{H}$ NMR (400 MHz, $\left.\mathrm{CDCl}_{3}\right): \delta 7.55-7.52(\mathrm{~m}, 2 \mathrm{H}), 7.33(\mathrm{~d}, J=7.6 \mathrm{~Hz}, 2 \mathrm{H}), 7.20(\mathrm{~s}, 2 \mathrm{H}), 2.53-2.49$ $(\mathrm{m}, 4 \mathrm{H}), 1.28-1.23(\mathrm{~m}, 24 \mathrm{H})$. 


\section{Optimization of Reaction Conditions}

Table S1. Screening of Ligands ${ }^{a}$
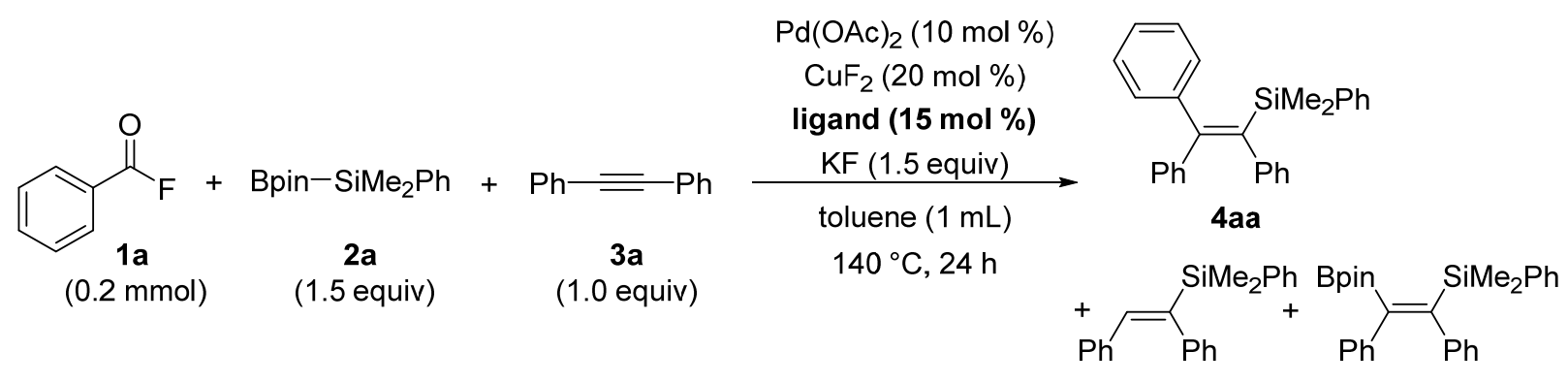

$5 a$

$6 a$

\begin{tabular}{|c|c|c|c|c|c|}
\hline \multirow{2}{*}{ entry } & \multirow{2}{*}{ ligand } & \multicolumn{4}{|c|}{ yield $(\%)^{b}$} \\
\hline & & 3a & $4 \mathbf{a a}$ & $5 \mathbf{a}$ & 6a \\
\hline 1 & DPPE & 27 & 1 & 41 & 7 \\
\hline 2 & DCYPE & 17 & 4 & 14 & 38 \\
\hline 3 & & 28 & 4 & 22 & 1 \\
\hline 4 & $\mathrm{PPh}_{3}(30 \mathrm{~mol} \%)$ & 1 & 0 & 22 & 52 \\
\hline 5 & $\mathrm{PCy}_{3}(30 \mathrm{~mol} \%)$ & 1 & 1 & 46 & 28 \\
\hline 6 & & 40 & $<1$ & 20 & 3 \\
\hline
\end{tabular}

${ }^{a}$ Reactions were carried out with $\mathbf{1 a}(0.2 \mathrm{mmol}), \mathbf{2 a}(0.3 \mathrm{mmol}, 1.5$ equiv $), \mathbf{3 a}\left(0.2 \mathrm{mmol}, 1.0\right.$ equiv), $\mathrm{Pd}(\mathrm{OAc})_{2}(0.02$ mmol, $10 \mathrm{~mol} \%), \mathrm{CuF}_{2}(20 \mathrm{~mol} \%)$, Ligand (0.03 mmol, $15 \mathrm{~mol} \%$ ), and $\mathrm{KF}\left(1.5\right.$ equiv) in toluene $(1 \mathrm{~mL})$ at $140{ }^{\circ} \mathrm{C}$ for $24 \mathrm{~h} .{ }^{b} \mathrm{GC}$ yields using n-dodecane as an internal standard. 
Table S2. Screening of Copper Catalyst ${ }^{a}$
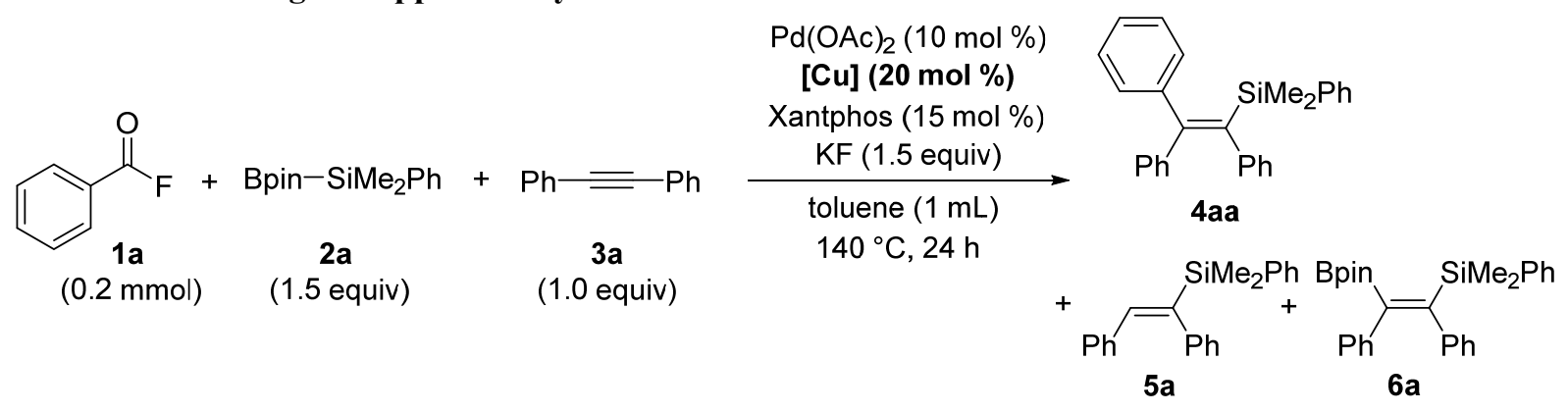

\begin{tabular}{|c|c|c|c|c|c|}
\hline \multirow{2}{*}{ entry } & \multirow{2}{*}[\mathrm{Cu}]{ catalyst } & \multicolumn{4}{|c|}{ yield $(\%)^{b}$} \\
\hline & & $3 \mathbf{a}$ & $4 \mathbf{a a}$ & $5 a$ & $6 a$ \\
\hline 1 & $\mathrm{CuF}_{2}$ & 28 & 4 & 22 & 1 \\
\hline 2 & $\mathrm{CuCl}$ & 40 & 13 & 24 & 2 \\
\hline 3 & $\mathrm{CuI}$ & 81 & 0 & 11 & $<1$ \\
\hline 4 & $\mathrm{Cu}(\mathrm{OAc})$ & 14 & 3 & 37 & $<1$ \\
\hline 5 & $\mathrm{Cu}(\mathrm{OAc})_{2}$ & 19 & 2 & 35 & $<1$ \\
\hline 6 & $\mathrm{CuBr} \cdot \mathrm{SMe}_{2}$ & 68 & 2 & 13 & 0 \\
\hline 7 & $\mathrm{CuTC}$ & 31 & 7 & 23 & 2 \\
\hline 8 & $\mathrm{IPrCuCl}(10 \mathrm{~mol} \%)$ & 26 & 24 & 23 & 2 \\
\hline 9 & $\mathrm{SIPrCuCl}(10 \mathrm{~mol} \%)$ & 58 & 8 & 14 & $<1$ \\
\hline 10 & $\operatorname{IPrCu}\left(\mathrm{O}^{t} \mathrm{Bu}\right)(10 \mathrm{~mol} \%)$ & 23 & 17 & 26 & $<1$ \\
\hline 11 & $\mathrm{IXyCuCl}(10 \mathrm{~mol} \%)$ & 2 & 27 & 20 & 8 \\
\hline 12 & IPrCuOTf (10 mol \%) & 50 & 37 & 13 & 0 \\
\hline $13^{c}$ & IPrCuOTf (10 mol \%) & 36 & 48 & 11 & 1 \\
\hline $14^{d}$ & $\operatorname{IPrCuOTf}(5 \mathrm{~mol} \%)$ & 39 & 42 & 11 & 3 \\
\hline
\end{tabular}

${ }^{a}$ Reactions were carried out with $\mathbf{1 a}(0.2 \mathrm{mmol}), \mathbf{2 a}(0.3 \mathrm{mmol}, 1.5$ equiv $), 3 \mathbf{3 a}(0.2 \mathrm{mmol}, 1.0$ equiv $), \operatorname{Pd}(\mathrm{OAc})_{2}$ (0.02 mmol, $10 \mathrm{~mol} \%$ ), [Cu] (20 mol \%), Xantphos (0.03 mmol, $15 \mathrm{~mol} \%$ ), and KF (1.5 equiv) in toluene (1 $\mathrm{mL}$ ) at $140{ }^{\circ} \mathrm{C}$ for $24 \mathrm{~h} .{ }^{b} \mathrm{GC}$ yields using $\mathrm{n}$-dodecane as an internal standard. ${ }^{c} \mathrm{Pd}(\mathrm{OAc})_{2}(5 \mathrm{~mol} \%)$, Xantphos (10 mol \%). ${ }^{d} \mathrm{Pd}(\mathrm{OAc})_{2}(2.5 \mathrm{~mol} \%)$, Xantphos (5 mol \%). 
Table S3. Screening of Base ${ }^{a}$
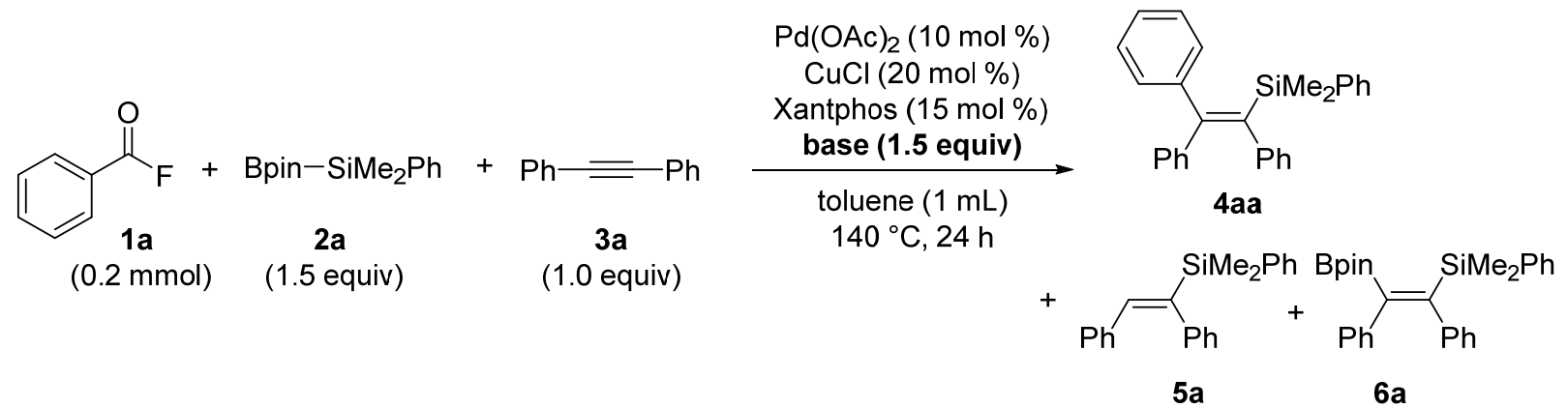

\begin{tabular}{|c|c|c|c|c|c|}
\hline \multirow{2}{*}{ entry } & \multirow{2}{*}{ base } & \multicolumn{4}{|c|}{ yield $(\%)^{b}$} \\
\hline & & $3 \mathbf{a}$ & $4 \mathbf{a a}$ & $5 \mathbf{a}$ & $6 \mathbf{a}$ \\
\hline 1 & $\mathrm{CsF}$ & 19 & $<1$ & 39 & $<1$ \\
\hline 2 & LiOMe & 42 & 0 & 5 & 0 \\
\hline 3 & $\mathrm{~K}_{2} \mathrm{CO}_{3}$ & 40 & 5 & 29 & $<1$ \\
\hline 4 & $\mathbf{K F}$ & 40 & 13 & 24 & 2 \\
\hline
\end{tabular}

${ }^{a}$ Reactions were carried out with $\mathbf{1 a}(0.2 \mathrm{mmol}, 1$ equiv), 2a ( $0.3 \mathrm{mmol}, 1.5$ equiv), 3a ( $0.2 \mathrm{mmol}, 1.0$ equiv), $\mathrm{Pd}(\mathrm{OAc})_{2}(0.02 \mathrm{mmol}, 10 \mathrm{~mol} \%), \mathrm{CuCl}(20 \mathrm{~mol} \%)$, Xantphos (0.03 mmol, $\left.15 \mathrm{~mol} \%\right)$, and base (1.5 equiv) in toluene $(1 \mathrm{~mL})$ at $140{ }^{\circ} \mathrm{C}$ for $24 \mathrm{~h} .{ }^{b} \mathrm{GC}$ yields using n-dodecane as an internal standard. 
Table S4. Screening of the Palladium Source ${ }^{a}$
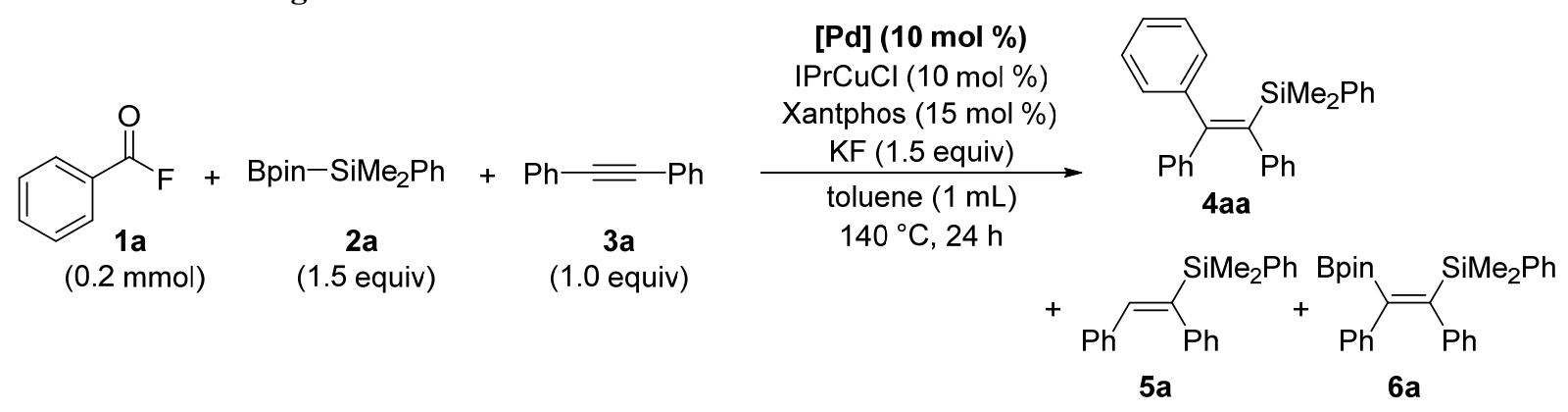

\begin{tabular}{lllrrrr}
\hline & & \multicolumn{5}{c}{ yield $(\%)^{b}$} \\
\cline { 3 - 6 } entry & {$[\mathbf{P d}]$} & $\mathbf{3 a}$ & $\mathbf{4 a a}$ & $\mathbf{5 a}$ & $\mathbf{6 a}$ \\
\hline 1 & $\mathrm{PdCl}_{2}$ & 45 & 5 & 15 & 3 \\
2 & $\mathrm{Pd}(\mathrm{acac})_{2}$ & 73 & 4 & 8 & $<1$ \\
3 & $\mathrm{Pd}_{2}(\mathrm{dba})_{3}(5 \mathrm{~mol} \%)$ & 74 & 13 & 12 & 2 \\
4 & $\mathbf{P d}(\mathbf{O A c})_{2}$ & $\mathbf{2 6}$ & $\mathbf{2 4}$ & $\mathbf{2 3}$ & $\mathbf{2}$ \\
\hline
\end{tabular}

${ }^{a}$ Reactions were carried out with $\mathbf{1 a}(0.2 \mathrm{mmol}, 1$ equiv), $\mathbf{2 a}(0.3 \mathrm{mmol}, 1.5$ equiv), 3a ( $0.2 \mathrm{mmol}, 1.0$ equiv), [Pd] (0.02 mmol, $10 \mathrm{~mol} \%), \operatorname{IPrCuCl}(10 \mathrm{~mol} \%)$, Xantphos (0.03 mmol, $15 \mathrm{~mol} \%)$, and $\mathrm{KF}$ (1.5 equiv) in toluene (1 $\mathrm{mL})$ at $140{ }^{\circ} \mathrm{C}$ for $24 \mathrm{~h} .{ }^{b} \mathrm{GC}$ yields using $\mathrm{n}$-dodecane as an internal standard. 
Table S5. Screening of the Solvent ${ }^{a}$
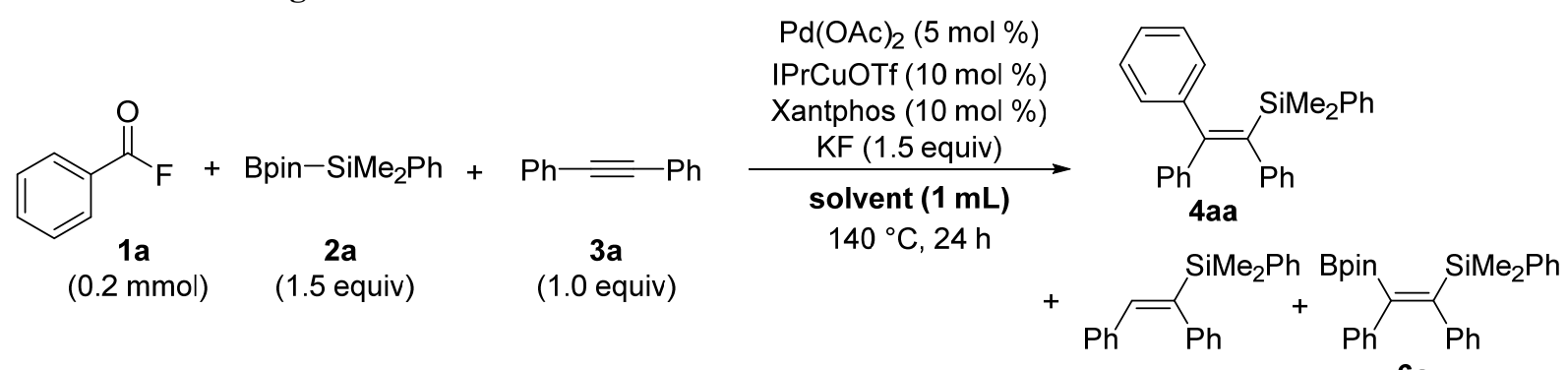

$5 a$

$6 a$

\begin{tabular}{|c|c|c|c|c|c|}
\hline \multirow{2}{*}{ entry } & \multirow{2}{*}{ Solvent (1 mL) } & \multicolumn{4}{|c|}{ yield $(\%)^{b}$} \\
\hline & & $3 \mathbf{a}$ & $4 \mathbf{a a}$ & $5 \mathbf{a}$ & $6 \mathbf{a}$ \\
\hline 1 & toluene & 36 & 48 & 11 & 1 \\
\hline 2 & toluene/1,4-dioxane $=4: 1$ & 38 & 35 & 13 & 1 \\
\hline 3 & toluene/1,4-dioxane $=1: 1$ & 2 & 58 & 9 & 1 \\
\hline 4 & toluene/1,4-dioxane $=1: 4$ & 34 & 37 & 14 & 1 \\
\hline 5 & 1,4-dioxane & 29 & 43 & 10 & 1 \\
\hline 6 & toluene/DMF = 1:1 & 46 & 8 & 19 & 1 \\
\hline 7 & toluene/NMP = 1:1 & 39 & 5 & 4 & 4 \\
\hline
\end{tabular}

${ }^{a}$ Reactions were carried out with $\mathbf{1 a}(0.2 \mathrm{mmol}, 1$ equiv), $2 \mathbf{a}(0.3 \mathrm{mmol}, 1.5$ equiv), $3 \mathbf{3}(0.2 \mathrm{mmol}, 1.0$ equiv), $\mathrm{Pd}(\mathrm{OAc})_{2}(0.01 \mathrm{mmol}, 5 \mathrm{~mol} \%)$, IPrCuOTf (10 mol \%), Xantphos (0.02 mmol, $\left.10 \mathrm{~mol} \%\right)$, and KF (1.5 equiv) in solvent $(1 \mathrm{~mL})$ at $140{ }^{\circ} \mathrm{C}$ for $24 \mathrm{~h} .{ }^{b} \mathrm{GC}$ yields using $\mathrm{n}$-dodecane as an internal standard. 
Table S6. Control Experiments ${ }^{a}$

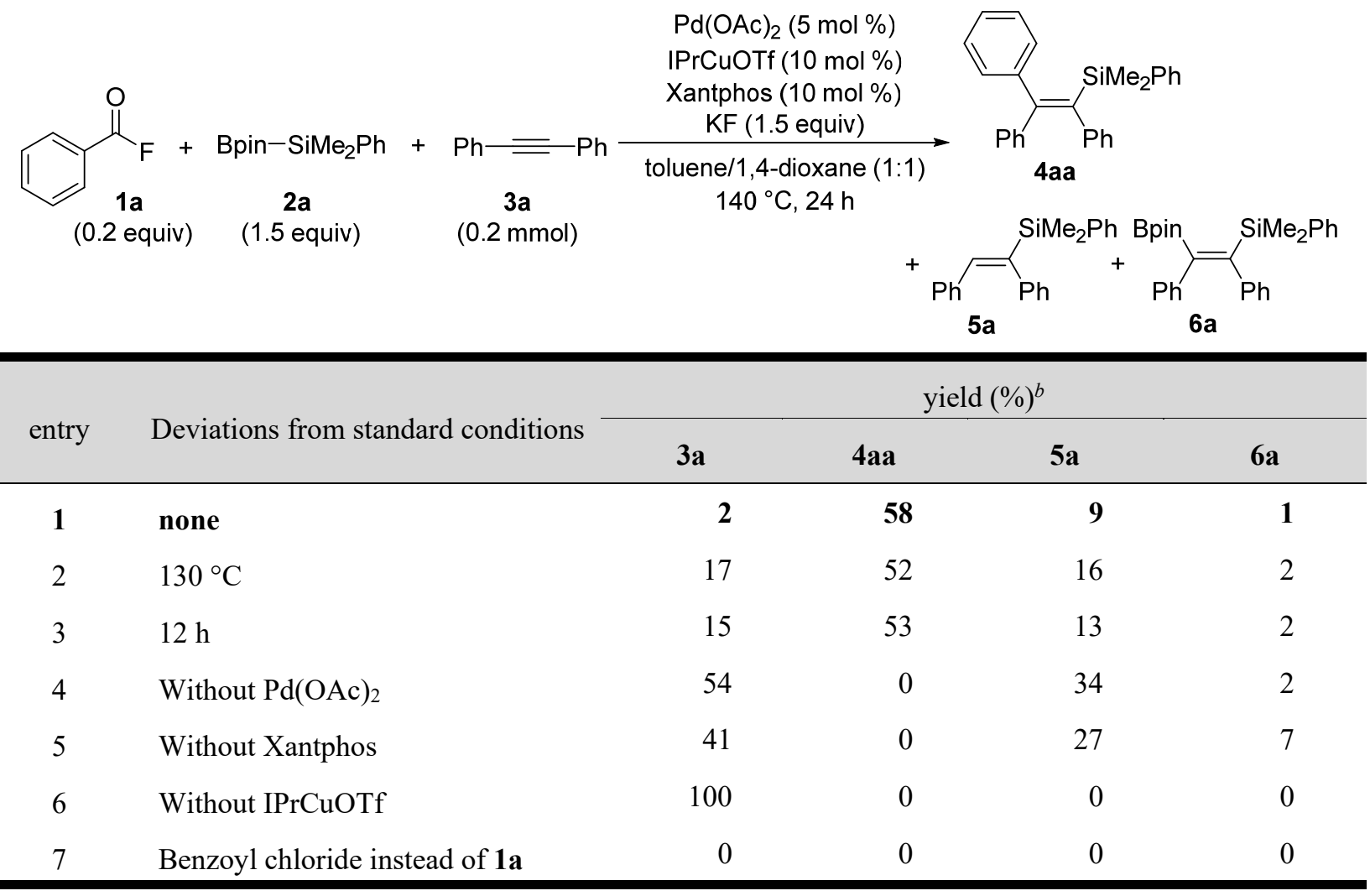

${ }^{a}$ Reactions were carried out with $\mathbf{1 a}\left(0.2 \mathrm{mmol}, 1\right.$ equiv), $\mathbf{2 a}\left(0.3 \mathrm{mmol}, 1.5\right.$ equiv), $\mathbf{3 a}\left(0.2 \mathrm{mmol}, 1.0\right.$ equiv), $\mathrm{Pd}(\mathrm{OAc})_{2}$ (0.01 mmol, $5 \mathrm{~mol} \%$ ), IPrCuOTf (10 mol \%), Xantphos (0.02 mmol, $10 \mathrm{~mol} \%$ ), and KF (1.5 equiv) in toluene/1,4dioxane $(1 \mathrm{~mL})$ at $140{ }^{\circ} \mathrm{C}$ for $24 \mathrm{~h} .{ }^{b} \mathrm{GC}$ yields using $\mathrm{n}$-dodecane as an internal standard. 
Table S7. Screening of Amounts of 1a and 2a

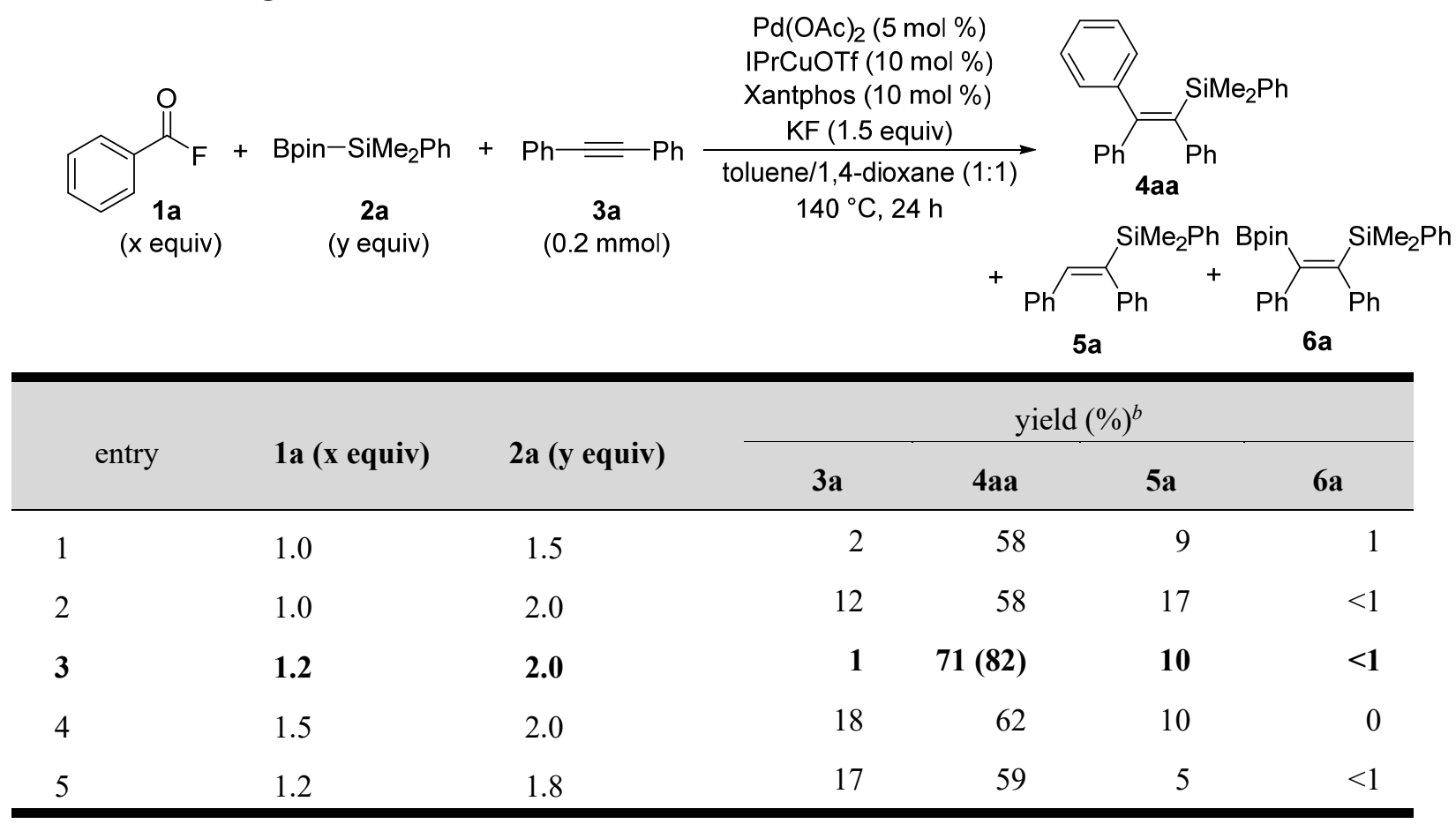

${ }^{a}$ Reactions were carried out with $1 \mathbf{a}$ (x equiv), 2 a (y equiv), $3 \mathbf{3}(0.2 \mathrm{mmol}), \operatorname{Pd}(\mathrm{OAc})_{2}(0.01 \mathrm{mmol}, 5 \mathrm{~mol} \%)$, IPrCuOTf (10 mol \%), Xantphos (0.02 mmol, $10 \mathrm{~mol} \%)$, and KF (1.5 equiv) in toluene/1,4-dioxane (1 mL) at $140{ }^{\circ} \mathrm{C}$ for $24 \mathrm{~h} . \quad{ }^{b} \mathrm{GC}$ yields using n-dodecane as an internal standard. An Isolated yield was shown in parentheses. 


\section{Experimental Procedures and Spectroscopic Data for the Products 4}

4.1 General Procedure for Palladium/Copper-Cocatalyzed Decarbonylative Arylsilylation of Internal Alkynes 3 with Acyl Fluorides 1 and Silylborane 2.

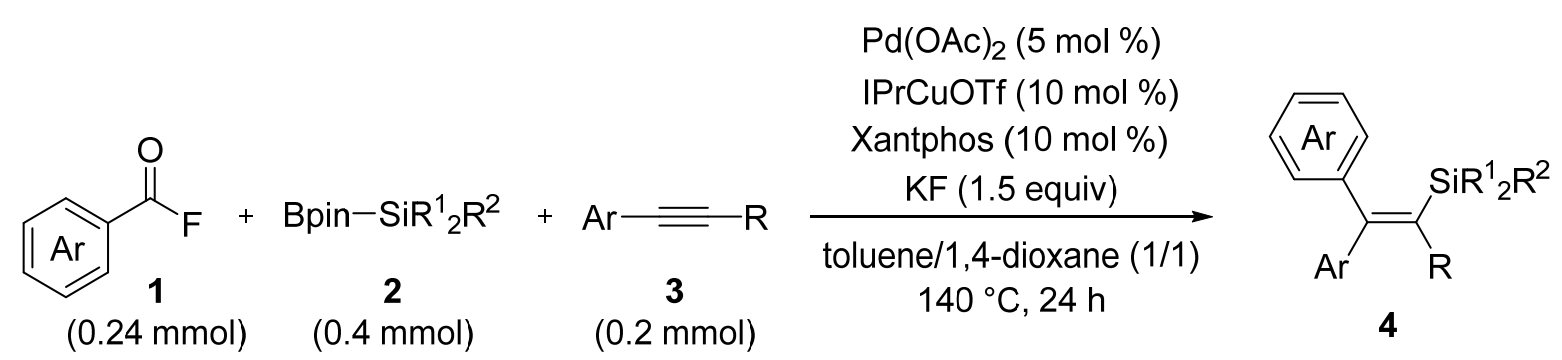

An oven-dried $20 \mathrm{~mL}$ of Schlenk tube containing a magnetic stirring bar was charged with $\mathrm{Pd}(\mathrm{OAc})_{2}(2.2 \mathrm{mg}$, $0.01 \mathrm{mmol}, 5 \mathrm{~mol} \%$ ), Xantphos (11.6 mg, $0.02 \mathrm{mmol}, 10 \mathrm{~mol} \%)$, IPrCuOTf(12 mg, $0.02 \mathrm{mmol}, 10 \mathrm{~mol} \%)$, KF (17.4 mg, $0.3 \mathrm{mmol}, 1.5$ equiv), toluene $(0.5 \mathrm{~mL})$, and 1,4-dioxane $(0.5 \mathrm{~mL})$ under argon, which was stirred for 30 seconds at room temperature. Then, acyl fluorides $1(0.24 \mathrm{mmol})$, silylborane $2(0.4 \mathrm{mmol})$, and internal alkynes $3(0.2 \mathrm{mmol})$ were added. The mixture was heated at $140{ }^{\circ} \mathrm{C}$ in a heating block with stirring for $24 \mathrm{~h}$. After being at room temperature, the mixture was quenched with saturated $\mathrm{NH}_{4} \mathrm{Cl}$ and then aqueous solution was extracted with diethyl ether. The combined organic phase was dried over anhydrous $\mathrm{MgSO}_{4}$, and evaporated under vacuum to remove the volatiles. The residue was purified by column chromatography (EtOAc or $\mathrm{CH}_{2} \mathrm{Cl}_{2}$ /hexane) on silica gel to afford the corresponding desired products 4 .

\subsection{Spectroscopic Data for the Products}

Dimethyl(phenyl)(1,2,2-triphenylethenyl)silane (4aa)<smiles>C[AsH2]C(=C(c1ccccc1)c1ccccc1)c1ccccc1</smiles>

4 aa

White solid. $\quad R_{\mathrm{f}}=0.18$ (hexane). Isolated yield was $82 \%(65.2 \mathrm{mg}){ }^{1} \mathrm{H} \mathrm{NMR}\left(400 \mathrm{MHz}, \mathrm{CDCl}_{3}\right): \delta 7.36-$ $7.16(\mathrm{~m}, 10 \mathrm{H}), 7.12-7.08(\mathrm{~m}, 2 \mathrm{H}), 7.04-6.92(\mathrm{~m}, 8 \mathrm{H}), 0.00(\mathrm{~s}, 6 \mathrm{H}) ;{ }^{13} \mathrm{C}\left\{{ }^{1} \mathrm{H}\right\} \mathrm{NMR}\left(101 \mathrm{MHz}, \mathrm{CDCl}_{3}\right): \delta 155.0$, $144.0,143.9,143.3,142.5,140.3,134.0,129.9$, 129.8, 129.6, 128.6, 128.0, 127.6, 127.5, 127.4, 127.3, 126.2, 125.1, -0.72. FT-IR (cm-1): 3062 (s), 3022 (s), 2969 (s), 2964 (s), 1596 (s), 1580 (s), 1570 (s), 1488 (s), 1443 
(s), 1426 (s), 1254 (s), 1248 (s), 1111 (s), 1075 (s), 1068 (s), 1028 (s), 1002 (s), 986 (s), 978 (s), 922 (s), 855 (s), 833 (s), 823 (s), 808 (s), 776 (s). Anal. Calcd for $\mathrm{C}_{28} \mathrm{H}_{26} \mathrm{Si}$ : C, 86.10; H, 6.71\%. Found: C, 86.05; H, 6.75\%.

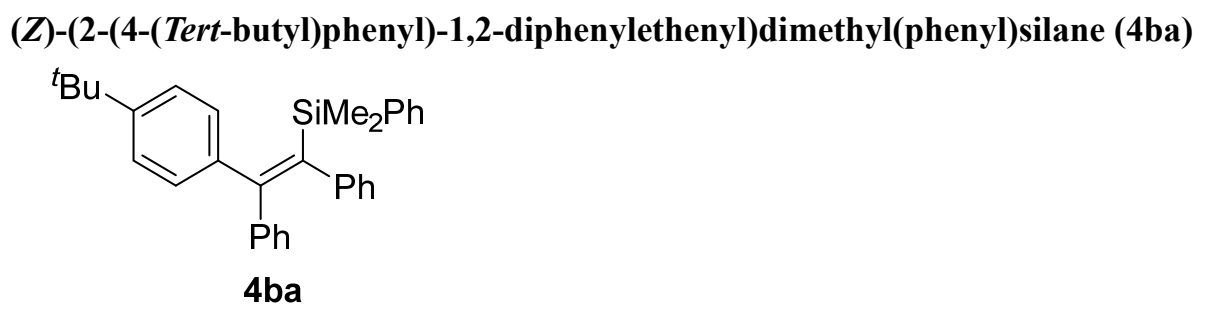

Colorless oil. $\quad R_{\mathrm{f}}=0.24$ (dichloromethane/hexane $\left.=1 / 10\right)$. Isolated yield was $45 \%(40.4 \mathrm{mg}) . \quad Z: E=13: 1$. ${ }^{1} \mathrm{H}$ NMR (400 MHz, $\mathrm{CDCl}_{3}$ ): (Z)-isomer (major); $\delta$ 7.35-7.24 (m, 5H), 7.21-7.18 (m, 2H), 7.12-7.06 (m, 4H), 7.04-6.93 (m, 8H), $1.32(\mathrm{~s}, 9 \mathrm{H}), 0.02(\mathrm{~s}, 6 \mathrm{H})$; (E)-isomer (minor); $\delta 7.35-7.24(\mathrm{~m}, 5 \mathrm{H}), 7.21-7.18(\mathrm{~m}, 2 \mathrm{H}), 7.12-$ $7.06(\mathrm{~m}, 4 \mathrm{H}), 7.04-6.93(\mathrm{~m}, 8 \mathrm{H}), 1.17(\mathrm{~s}, 9 \mathrm{H}),-0.02(\mathrm{~s}, 6 \mathrm{H}) ;{ }^{13} \mathrm{C}\left\{{ }^{1} \mathrm{H}\right\} \mathrm{NMR}\left(101 \mathrm{MHz}, \mathrm{CDCl}_{3}\right): \delta 155.4,150.1$, $144.1,143.5,142.3,140.9,140.3,133.9,129.9,129.6,129.2,128.5,127.5_{2}, 127.4_{5}, 127.3,126.1,125.0,124.7$, 34.6, 31.5, -0.7. FT-IR (cm-1): 3070 (s), 3064 (s), 2963 (s), 2870 (s), 1510 (s), 1487 (s), 1420 (s), 1342 (s), 1219 (s), 1210 (s), 1097 (s), 830 (s), 822 (s), 810 (s), 806 (s), 713 (s). Anal. Calcd for $\mathrm{C}_{32} \mathrm{H}_{34} \mathrm{Si}: \mathrm{C}, 86.04$; H, 7.67\%. Found: C, 85.92; H, 7.52\%.

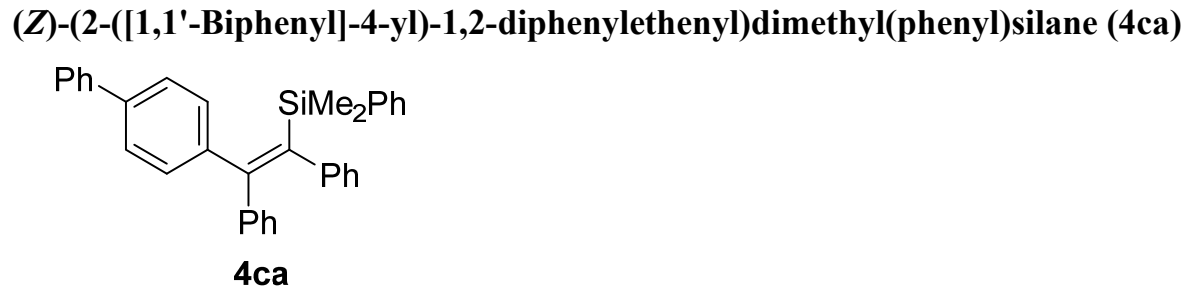

White solid. $\quad R_{\mathrm{f}}=0.25$ (dichloromethane/hexane =1/10). Isolated yield was $51 \%(48.0 \mathrm{mg}) . \quad{ }^{1} \mathrm{H} \mathrm{NMR}(400$ $\left.\mathrm{MHz}, \mathrm{CDCl}_{3}\right): \delta$ 7.62-7.57 (m, 2H), 7.47-7.18 (m, 12H), 7.14-7.10 (m, 2H), 7.05-6.95 (m, 8H), 0.06 (s, 6H); ${ }^{13} \mathrm{C}\left\{{ }^{1} \mathrm{H}\right\} \mathrm{NMR}\left(101 \mathrm{MHz}, \mathrm{CDCl}_{3}\right): \delta 155.0,144.0,143.3,143.0,142.9,141.0,140.2,139.9_{4}, 133.91,130.1,129.8$, 129.6, 128.9, 128.6, 127.6, 127.5, 127.4 $3,127.3_{7}, 127.2,126.6,126.3,125.2,-0.5$. FT-IR $\left(\mathrm{cm}^{-1}\right): 3063(\mathrm{~s}), 3024$ (s), 2960 (s), 1599 (s), 1487 (s), 1439 (s), 1427 (s), 1401 (s), 1254 (s), 1248 (s), 1111 (s), 1070 (s), 978 (s), 857 (s), 832 (s), 818 (s), 809 (s), 776 (s), 765 (s), 734 (s), 719 (s). Anal. Calcd for $\mathrm{C}_{34} \mathrm{H}_{30} \mathrm{Si}: \mathrm{C}$, 87.50; H, 6.48\%. Found: C, 87.15; H, 6.34\%.

(Z)-(2-(3-Methoxyphenyl)-1,2-diphenylethenyl)dimethyl(phenyl)silane (4da) ${ }^{7}$ 
<smiles>COc1cccc(C(=C(c2ccccc2)c2ccccc2)c2ccccc2)c1</smiles>

4da

White solid. $\quad R_{\mathrm{f}}=0.22$ (dichloromethane/hexane $\left.=1 / 4\right)$. Isolated yield was 50\% $(43.0 \mathrm{mg}) . \quad Z: E=10: 1$. ${ }^{1} \mathrm{H}$ NMR (400 MHz, $\mathrm{CDCl}_{3}$ ): (Z)-isomer (major); $\delta$ 7.40-7.26 (m, 5H), 7.17-7.10 (m, 3H), 7.05-6.94 (m, 8H), 6.82-6.76 (m, 2H), 6.70-6.69 (m, 1H), $3.62(\mathrm{~s}, 3 \mathrm{H}), 0.05(\mathrm{~s}, 6 \mathrm{H})$; (E)-isomer (minor); $\delta$ 7.40-7.26 (m, 5H), 7.17$7.10(\mathrm{~m}, 3 \mathrm{H}), 7.05-6.94(\mathrm{~m}, 8 \mathrm{H}), 6.54-6.51(\mathrm{~m}, 3 \mathrm{H}), 3.53(\mathrm{~s}, 3 \mathrm{H}), 0.01(\mathrm{~s}, 6 \mathrm{H}) ;{ }^{13} \mathrm{C}\left\{{ }^{1} \mathrm{H}\right\} \mathrm{NMR}\left(101 \mathrm{MHz}, \mathrm{CDCl}_{3}\right)$ : $\delta 159.2,155.1,145.2,143.9,143.1,142.3,140.4,133.9,129.8,129.0,128.6,127.6_{1}, 127.5_{9}, 127.5_{2}, 127.4,126.2$, $125.2,122.3,115.0,113.2,55.1,-0.6$.

\section{(Z)-(1,2-Diphenyl-2-(4-(trifluoromethyl)phenyl)ethenyl)dimethyl(phenyl)silane (4ea)}<smiles>FC(F)(F)c1ccc(C(=C(c2ccccc2)c2ccccc2)c2ccccc2)cc1</smiles>

Colorless oil. $\quad R_{\mathrm{f}}=0.35$ (dichloromethane/hexane $\left.=1 / 10\right)$. Isolated yield was $50 \%(46.0 \mathrm{mg}) . \quad{ }^{1} \mathrm{H}$ NMR $\left(400 \mathrm{MHz}, \mathrm{CDCl}_{3}\right): \delta 7.42(\mathrm{~d}, J=8.0 \mathrm{~Hz}, 2 \mathrm{H}), 7.35-7.23(\mathrm{~m}, 7 \mathrm{H}), 7.19-7.15(\mathrm{~m}, 2 \mathrm{H}), 7.01-6.97(\mathrm{~m}, 6 \mathrm{H}), 6.97-$ $6.92(\mathrm{~m}, 2 \mathrm{H}), 0.10(\mathrm{~s}, 6 \mathrm{H}) ;{ }^{13} \mathrm{C}\left\{{ }^{1} \mathrm{H}\right\} \mathrm{NMR}\left(101 \mathrm{MHz}, \mathrm{CDCl}_{3}\right): \delta 153.8,147.3,144.0,143.5,142.5,139.5,133.7$, $129.9,129.6_{2}, 129.56,129.3\left(\mathrm{q}, J_{\mathrm{C}-\mathrm{F}}=32.6 \mathrm{~Hz}\right), 128.8,127.7_{3}, 127.7_{0}, 127.6,126.5,125.4,124.8$ (q, $J_{\mathrm{C}-\mathrm{F}}=3.8$ $\mathrm{Hz}), 124.3\left(\mathrm{q}, J_{\mathrm{C}-\mathrm{F}}=273.2 \mathrm{~Hz}\right),-0.6 ;{ }^{19} \mathrm{~F}\left\{{ }^{1} \mathrm{H}\right\} \mathrm{NMR}\left(376 \mathrm{MHz}, \mathrm{CDCl}_{3}, \mathrm{rt}\right): \delta-62.8 . \quad$ FT-IR $\left(\mathrm{cm}^{-1}\right): 3074(\mathrm{~s})$, 1615 (s), 1487 (s), 1438 (s), 1429 (s), 1404 (s), 1331 (s), 1327 (s), 1311 (s), 1258 (s), 1249 (s), 1164 (s), 1154 (s), 1128 (s), 1117 (s), 1107 (s), 1068 (s), 1018 (s), 859 (s), 843 (s), 832 (s), 818 (s), 805 (s), 775 (s), 760 (s), 738 (s), 727 (s), 703 (s). Anal. Calcd for $\mathrm{C}_{29} \mathrm{H}_{25} \mathrm{~F}_{3} \mathrm{Si}$ : C, 75.95; H, 5.49\%. Found: C, 75.91; H, 5.42\%.

\section{(Z)-4-(2-(Dimethyl(phenyl)silyl)-1,2-diphenylethenyl)benzonitrile (4fa)}<smiles>N#Cc1ccc(C(=C(c2ccccc2)c2ccccc2)c2ccccc2)cc1</smiles>

4fa 
Colorless oil. $\quad R_{\mathrm{f}}=0.48$ (dichloromethane/hexane $\left.=1 / 1\right)$. Isolated yield was 51\% (42.1 mg). $Z: E=3: 1$. ${ }^{1} \mathrm{H}$ NMR (400 MHz, $\mathrm{CDCl}_{3}$ ): (Z)-isomer (major); $\delta$ 7.42-7.39 (m, 2H), 7.32-7.24 (m, 6H), 7.20-6.96 (m, 9H), 6.89-6.86 (m, 2H), $0.07(\mathrm{~s}, 6 \mathrm{H}) ;(E)$-isomer (minor); $\delta$ 7.32-7.24 (m, 8H), 7.20-6.96 (m, 9H), 6.89-6.86 (m, 2H), $0.01(\mathrm{~s}, 6 \mathrm{H}) ;{ }^{13} \mathrm{C}\left\{{ }^{1} \mathrm{H}\right\} \mathrm{NMR}\left(101 \mathrm{MHz}, \mathrm{CDCl}_{3}\right): \delta 153.3,148.4,144.5,143.2,142.0,139.3,133.7,131.7,130.4$, 129.5, 129.4, 128.9, 127.8, 127.7, 127.6, 126.7, 125.5, 119.0, 110.9, -0.7. FT-IR (cm-1): 3068 (s), 3053 (s), 3024 (s), 2955 (s), 2228 (s), 1598 (s), 1493 (s), 1420 (s), 1404 (s), 1403 (s), 1226 (s), 1221 (s), 1111 (s), 983 (s), 858 (s), 834 (s), 811 (s), 736 (s), 701 (s). Anal. Calcd for $\mathrm{C}_{29} \mathrm{H}_{25} \mathrm{NSi}: \mathrm{C}, 83.81$; H, 6.06; N, 3.37\%. Found: C, $83.83 ; \mathrm{H}, 5.81 ; \mathrm{N}, 3.36 \%$.

\section{(Z)-Methyl 4-(2-(dimethyl(phenyl)silyl)-1,2-diphenylethenyl)benzoate (4ga)}<smiles>COC(=O)c1ccc(C([AsH2]c2ccccc2)=C(c2ccccc2)c2ccccc2)cc1</smiles>

White solid. $\quad R_{\mathrm{f}}=0.48$ (dichloromethane/hexane $\left.=1 / 1\right)$. Isolated yield was $73 \%(65.7 \mathrm{mg}) . \quad Z: E=5: 1 . \quad{ }^{1} \mathrm{H}$ NMR (400 MHz, $\left.\mathrm{CDCl}_{3}\right)$ : (Z)-isomer (major); $\delta$ 7.88-7.85 (m, 2H), 7.32-7.21 (m, 6H), 7.12-7.09 (m, 3H), 7.03$6.88(\mathrm{~m}, 8 \mathrm{H}), 3.92(\mathrm{~s}, 3 \mathrm{H}), 0.07(\mathrm{~s}, 6 \mathrm{H}) ;(E)$-isomer (minor); $\delta$ 7.67-7.65 (m, 2H), 7.32-7.21 (m, 9H), 7.03-6.88 (m, 8H), $3.80(\mathrm{~s}, 3 \mathrm{H}), 0.01(\mathrm{~s}, 6 \mathrm{H}) ;{ }^{13} \mathrm{C}\left\{{ }^{1} \mathrm{H}\right\} \mathrm{NMR}\left(101 \mathrm{MHz}, \mathrm{CDCl}_{3}\right): \delta 167.1,154.1,148.7,143.6,143.5,142.6$, $139.7,133.9,129.8,129.6_{8}, 129.6_{6}, 129.5_{7}, 129.3,128.8,127.7,127.6,127.5,126.5,125.4,52.2,-0.7$. FT-IR (cm $\left.{ }^{-1}\right): 3065$ (s), 3048 (s), 3020 (s), 2951 (s), 1723 (s), 1607 (s), 1594 (s), 1579 (s), 1564 (s), 1488 (s), 1435 (s), 1426 (s), 1402 (s), 1308 (s), 1287 (s), 1271 (s), 1260 (s), 1250 (s), 1191 (s), 1175 (s), 1157 (s), 1113 (s), 1104 (s), 1073 (s), 1020 (s), 980 (s), 966 (s), 872 (s), 855 (s), 836 (s), 827 (s), 816 (s), 807 (s), 780 (s), 766 (s), 753 (s), 742 (s), 728 (s), 714 (s). Anal. Calcd for $\mathrm{C}_{30} \mathrm{H}_{28} \mathrm{O}_{2}$ Si: C, 80.32; H, 6.29\%. Found: C, 80.34; H, 6.30\%.

\section{(Z)-Phenyl 4-(2-(dimethyl(phenyl)silyl)-1,2-diphenylethenyl)benzoate (4ha)}<smiles>C[AsH2]C(=C(c1ccccc1)c1ccccc1)c1ccccc1</smiles>

4ha 
White solid. $\quad R_{\mathrm{f}}=0.61$ (dichloromethane/hexane $=1 / 1$ ). Isolated yield was 50\% (51.1 mg). $Z: E=5: 1 . \quad{ }^{1} \mathrm{H}$ NMR (400 MHz, $\mathrm{CDCl}_{3}$ ): (Z)-isomer (major); $\delta$ 8.06-8.04 (m, 2H), 7.49-7.44 (m, 2H), 7.38-7.25 (m, 10H), 7.18$6.94(\mathrm{~m}, 10 \mathrm{H}), 0.10(\mathrm{~s}, 6 \mathrm{H}) ;(E)$-isomer (minor); $\delta$ 7.87-7.85 (m, 2H), 7.49-7.44 (m, 2H), 7.38-7.25 (m, $10 \mathrm{H})$, 7.18-6.94 (m, 10H), $0.06(\mathrm{~s}, 6 \mathrm{H}) ;{ }^{13} \mathrm{C}\left\{{ }^{1} \mathrm{H}\right\} \mathrm{NMR}\left(101 \mathrm{MHz}, \mathrm{CDCl}_{3}\right): \delta 165.1,154.0,151.1,149.4,143.7,143.4$, $142.5,139.6,133.9,130.0,129.9,129.7,129.6_{3}, 129.57,128.8,128.2,127.7,127.64,127.56,126.5,126.0,125.4$, 121.8,-0.6. FT-IR (cm-1): 3066 (s), 3048 (s), 1730 (s), 1593 (s), 1492 (s), 1428 (s), 1272 (s), 1252 (s), 1192 (s), 1176 (s), 1073 (s), 880 (s), 721 (s), 700 (s). Anal. Calcd for $\mathrm{C}_{35} \mathrm{H}_{30} \mathrm{O}_{2} \mathrm{Si}: \mathrm{C}, 82.31$; H, 5.92\%. Found: C, 82.36; $\mathrm{H}, 5.86 \%$.

\section{(Z)-(2-(4-Fluorophenyl)-1,2-diphenylethenyl)dimethyl(phenyl)silane (4ia) ${ }^{7}$}<smiles>Fc1ccc(C(=C(c2ccccc2)c2ccccc2)c2ccccc2)cc1</smiles>

4ia

Colorless oil. $\quad R_{\mathrm{f}}=0.56$ (dichloromethane/hexane $\left.=1 / 10\right)$. Isolated yield was $46 \%(37.3 \mathrm{mg}) . \quad Z: E=6: 1$. ${ }^{1} \mathrm{H}$ NMR (400 MHz, $\mathrm{CDCl}_{3}$ ): (Z)-isomer (major); $\delta$ 7.35-7.17 (m, 5H), 7.15-6.85 (m, 14H), 0.06 (s, 6H); $(E)$ isomer (minor); $\delta$ 7.35-7.17 (m, 5H), 7.15-6.85 (m, 14H), $0.01(\mathrm{~s}, 6 \mathrm{H}) ;{ }^{13} \mathrm{C}\left\{{ }^{1} \mathrm{H}\right\} \mathrm{NMR}\left(101 \mathrm{MHz}, \mathrm{CDCl}_{3}\right): \delta$ $162.2\left(\mathrm{~d}, J_{\mathrm{C}-\mathrm{F}}=247.3 \mathrm{~Hz}\right), 154.2,143.8,143.3,143.1,140.1 ., 139.9\left(\mathrm{~d}, J_{\mathrm{C}-\mathrm{F}}=3.4 \mathrm{~Hz}\right), 133.8,131.4\left(\mathrm{~d}, J_{\mathrm{C}-\mathrm{F}}=8.0\right.$ $\mathrm{Hz}), 129.8,129.6,128.7,127.7,127.6,127.4,126.3,125.3,114.8\left(\mathrm{~d}, J_{\mathrm{C}-\mathrm{F}}=21.3 \mathrm{~Hz}\right),-0.6 .{ }^{19} \mathrm{~F}\left\{{ }^{1} \mathrm{H}\right\} \mathrm{NMR}(376$ $\left.\mathrm{MHz}, \mathrm{CDCl}_{3}, \mathrm{rt}\right): \delta-115.6$.

\section{(Z)-(1,2-Diphenyl-2-(2-methylphenyl)ethenyl)dimethyl(phenyl)silane (4ja)}

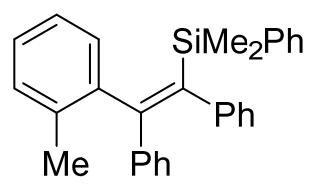

4ja

White solid. $\quad R_{\mathrm{f}}=0.48$ (dichloromethane/hexane = 1/10). Isolated yield was 53\% (43 mg). ${ }^{1} \mathrm{H}$ NMR (400 $\left.\mathrm{MHz}_{2} \mathrm{CDCl}_{3}\right): \delta 7.31-7.27(\mathrm{~m}, 1 \mathrm{H}), 7.26-7.08(\mathrm{~m}, 9 \mathrm{H}), 7.06-7.01(\mathrm{~m}, 2 \mathrm{H}), 6.96-6.88(\mathrm{~m}, 7 \mathrm{H}), 2.06(\mathrm{~s}, 3 \mathrm{H}), 0.03$ $(\mathrm{s}, 3 \mathrm{H}),-0.09(\mathrm{~s}, 3 \mathrm{H}) ;{ }^{13} \mathrm{C}\left\{{ }^{1} \mathrm{H}\right\} \mathrm{NMR}\left(101 \mathrm{MHz}, \mathrm{CDCl}_{3}\right): \delta 154.0,143.7,142.9,142.7,141.8,139.6,136.6,134.0$, $130.7,130.2,130.0_{4}, 130.0_{0}, 128.6,127.8,127.5_{5}, 127.4_{9}, 127.4_{6}, 127.2,126.2,125.3,125.2,20.0,-0.5,-2.0$. 
The two methyl groups were observed inequivalent, probably due to the restriction of the free rotation of the carbon-silicon bond. FT-IR (cm $\left.{ }^{-1}\right): 3021$ (s), 3012 (s), 2873 (s), 1496 (s), 1432 (s), 1321 (s), 1316 (s), 1111 (s), 831 (s), 816 (s), 810 (s), 718 (s), 698 (s). Anal. Calcd for $\mathrm{C}_{29} \mathrm{H}_{28} \mathrm{Si}$ : C, 86.08; H, 6.98\%. Found: C, 86.02; H, $6.98 \%$.

\section{(Z)-(2-([1,1'-Biphenyl]-2-yl)-1,2-diphenylethenyl)dimethyl(phenyl)silane (4ka)}<smiles>C[AsH2](C(=C(c1ccccc1)c1ccccc1)c1ccccc1)c1ccccc1</smiles>

4ka

White solid. $\quad R_{\mathrm{f}}=0.43$ (dichloromethane/hexane $\left.=1 / 10\right)$. Isolated yield was $50 \%(46.3 \mathrm{mg}) . \quad{ }^{1} \mathrm{H}$ NMR (400 $\left.\mathrm{MHz}, \mathrm{CDCl}_{3}\right): \delta$ 7.50-7.26 (m, 14H), 7.09-6.99 (m, 3H), 6.86-6.84 (m, 2H), 6.78-6.68 (m, 3H), 6.44-6.41 (m, 2H), 0.19 (s, 3H), 0.15 (s, 3H); ${ }^{13} \mathrm{C}\left\{{ }^{1} \mathrm{H}\right\}$ NMR (101 MHz, $\left.\mathrm{CDCl}_{3}\right): \delta 154.3,143.4,143.0,142.7,142.3,141.5$, 141.1, 139.8, 134.2, 131.4, 130.2, 130.0, 129.7, 129.4, 128.8, 128.2, 127.6 (x 2), 127.3, 126.8, 126.7, 126.4, $125.5,125.1,0.0,-2.1$. The two methyl groups were observed inequivalent, probably due to the restriction of the free rotation of the carbon-silicon bond. FT-IR ( $\left.\mathrm{cm}^{-1}\right): 3071$ (s), 3055 (s), 3045 (s), 2947 (s), 2919 (s), 1595 (s), 1483 (s), 1474 (s), 1440 (s), 1425 (s), 1260 (s), 1247 (s), 1112 (s), 855 (s), 836 (s), 826 (s), 804 (s), 782 (s), 773 (s), 766 (s), 757 (s), 744 (s), 737 (s), 728 (s), 706 (s). Anal. Calcd for $\mathrm{C}_{34} \mathrm{H}_{30} \mathrm{Si}$ : C, 87.50; H, 6.48\%. Found: C, $87.41 ; \mathrm{H}, 6.53 \%$.

\section{(Z)-(1,2-Diphenyl-2-(2-(trifluoromethyl)phenyl)ethenyl)dimethyl(phenyl)silane (4la)}<smiles>C[As](C(=C(c1ccccc1)c1ccccc1)c1ccccc1)c1ccccc1C(F)(F)F</smiles>

4la

Colorless oil. $\quad R_{\mathrm{f}}=0.41$ (dichloromethane/hexane $\left.=1 / 10\right)$. Isolated yield was $40 \%(36.9 \mathrm{mg}) . \quad{ }^{1} \mathrm{H} \mathrm{NMR}$ (400 MHz, $\mathrm{CDCl}_{3}$ ): $\delta 7.62(\mathrm{~d}, J=7.6 \mathrm{~Hz}, 1 \mathrm{H}), 7.53-7.50(\mathrm{~m}, 1 \mathrm{H}), 7.45-7.37(\mathrm{~m}, 3 \mathrm{H}), 7.34-7.24(\mathrm{~m}, 4 \mathrm{H}), 7.13-$ $7.04(\mathrm{~m}, 3 \mathrm{H}), 6.98-6.89(\mathrm{~m}, 7 \mathrm{H}), 0.05(\mathrm{~s}, 3 \mathrm{H}),-0.08(\mathrm{~s}, 3 \mathrm{H}) ;{ }^{13} \mathrm{C}\left\{{ }^{1} \mathrm{H}\right\}$ NMR (101 MHz, $\left.\mathrm{CDCl}_{3}\right): \delta 150.4,144.3$, $143.0,141.7\left(\mathrm{q}, J_{\mathrm{C}-\mathrm{F}}=5.5 \mathrm{~Hz}\right), 141.4,139.2,134.1,132.9,131.3,129.9,128.9\left(\mathrm{q}, J_{\mathrm{C}-\mathrm{F}}=32.9 \mathrm{~Hz}\right), 128.7,127.9$, 127.6, 127.5, 127.1, 126.8 (q, $\left.J_{\mathrm{C}-\mathrm{F}}=5.0 \mathrm{~Hz}\right), 126.4,125.4,124.3$ (q, $\left.J_{\mathrm{C}-\mathrm{F}}=275.5 \mathrm{~Hz}\right),-1.1,-2.1$. The two methyl groups were observed inequivalent, probably due to the restriction of the free rotation of the carbon- 
silicon bond. FT-IR (cm ${ }^{-1}$ ): 3068 (s), 3023 (s), 3011 (s), 2950 (s), 1498 (s), 1430 (s), 1410 (s), 1400 (s), 1314 (s), 1258 (s), 1170 (s), 1128 (s), 1109 (s), 1053 (s), 1035 (s), 820 (s), 814 (s), 735 (s). Anal. Calcd for $\mathrm{C}_{29} \mathrm{H}_{25} \mathrm{~F}_{3} \mathrm{Si}: \mathrm{C}, 75.95 ; \mathrm{H}, 5.49 \%$. Found: C, 76.23; H, 5.38\%.

\section{(Z)-(2-Mesityl-1,2-diphenylethenyl)dimethyl(phenyl)silane (4ma)}<smiles>Cc1cc(C)c(/C(=C(/[AsH2])c2ccccc2)c2ccccc2)c(C)c1</smiles>

Colorless oil. $\quad R_{\mathrm{f}}=0.48$ (dichloromethane/hexane $\left.=1 / 10\right) . \quad$ Isolated yield was $50 \%(43 \mathrm{mg}) . \quad{ }^{1} \mathrm{H}$ NMR (400 $\left.\mathrm{MHz}, \mathrm{CDCl}_{3}\right): \delta$ 7.34-7.26 (m, 5H), 7.15-7.08 (m, 3H), 6.96-6.93 (m, 7H), 6.87-6.86 (m, 2H), $2.34(\mathrm{~s}, 3 \mathrm{H}), 2.28$ (s, 6H), $0.00(\mathrm{~s}, 6 \mathrm{H}) ;{ }^{13} \mathrm{C}\left\{{ }^{1} \mathrm{H}\right\} \mathrm{NMR}\left(101 \mathrm{MHz}, \mathrm{CDCl}_{3}\right): \delta 151.4,144.3,142.3,141.3,140.0,139.1,136.7,136.2$, 134.3, 130.2, 130.0, 128.6, 128.5, 127.7, 127.4, 127.2, 126.2, 125.3, 21.3, 20.6, -1.8. FT-IR (cm $\left.{ }^{-1}\right): 3068(\mathrm{~s})$, 3052 (s), 2953 (s), 2916 (s), 1598 (s), 1497 (s), 1431 (s), 1420 (s), 1330 (s), 1326 (s), 1187 (s), 830 (s), 806 (s), 786 (s). Anal. Calcd for $\mathrm{C}_{31} \mathrm{H}_{32} \mathrm{Si}$ : C, 86.05; H, 7.45\%. Found: C, 86.02; H, 7.36\%.

\section{(Z)-Dimethyl(2-(naphthalen-1-yl)-1,2-diphenylethenyl)(phenyl)silane (4na)}<smiles>C[SiH](C(=C(c1ccccc1)c1ccccc1)c1ccccc1)c1ccccc1</smiles>

4na

White solid. $\quad R_{\mathrm{f}}=0.5$ (dichloromethane/hexane $\left.=1 / 10\right) . \quad$ Isolated yield was $46 \%(40.4 \mathrm{mg}) . \quad{ }^{1} \mathrm{H}$ NMR $(400$ $\left.\mathrm{MHz}, \mathrm{CDCl}_{3}\right): \delta 8.07-8.04(\mathrm{~m}, 1 \mathrm{H}), 7.81-7.76(\mathrm{~m}, 2 \mathrm{H}), 7.44-7.37(\mathrm{~m}, 4 \mathrm{H}), 7.22-7.19(\mathrm{~m}, 1 \mathrm{H}), 7.19-7.14(\mathrm{~m}, 6 \mathrm{H})$, 7.12-7.03 (m, 5H), 6.96-6.91 (m, 3H), -0.08 (s, 3H), -0.31 (s, 3H); ${ }^{13} \mathrm{C}\left\{{ }^{1} \mathrm{H}\right\} \mathrm{NMR}\left(101 \mathrm{MHz}, \mathrm{CDCl}_{3}\right): \delta 152.5$, $144.8,143.8,142.5,140.9,139.3,133.82,133.76,132.5,130.0,129.6,128.5,128.3,128.0,127.8,127.7,127.3$ (x 2), 126.5, 126.4, 126.1, 125.7, 125.3, 125.1, -0.7, -1.8. The two methyl groups were observed inequivalent, probably due to the restriction of the free rotation of the carbon-silicon bond. FT-IR ( $\left.\mathrm{cm}^{-1}\right): 3068$ (s), 3052 (s), 3027 (s), 1597 (s), 1440 (s), 1429 (s), 1247 (s), 1116 (s), 961 (s), 870 (s), 865 (s), 838 (s), 823 (s), 810 (s), 800 (s), 782 (s), 772 (s), 735 (s), 703 (s). Anal. Calcd for $\mathrm{C}_{32} \mathrm{H}_{28} \mathrm{Si}$ : C, 87.22; H, 6.40\%. Found: C, 87.15; H, 6.32\%.

\section{(Z)-Dimethyl(2-(naphthalen-2-yl)-1,2-diphenylethenyl)(phenyl)silane (4oa) ${ }^{7}$}


<smiles>C[AsH2]C(=C(c1ccccc1)c1ccccc1)c1ccccc1</smiles>

$40 a$

White solid. $\quad R_{\mathrm{f}}=0.29$ (dichloromethane/hexane $\left.=1 / 10\right)$. Isolated yield was 59\% (52.3 mg). ${ }^{1} \mathrm{H}$ NMR (400 $\left.\mathrm{MHz}, \mathrm{CDCl}_{3}\right): \delta 7.83-7.80(\mathrm{~m}, 1 \mathrm{H}), 7.68-7.62(\mathrm{~m}, 3 \mathrm{H}), 7.52-7.47(\mathrm{~m}, 2 \mathrm{H}), 7.33-7.25(\mathrm{~m}, 5 \mathrm{H}), 7.21-7.16(\mathrm{~m}, 3 \mathrm{H})$, 7.08-7.05 (m, 3H), 7.03-6.95 (m, 5H), $0.00(\mathrm{~s}, 6 \mathrm{H}) ;{ }^{13} \mathrm{C}\left\{{ }^{1} \mathrm{H}\right\}$ NMR (101 MHz, $\left.\mathrm{CDCl}_{3}\right): \delta 155.2,144.1,143.1$, 143.0, 141.5, 140.6, 133.9, 132.8, 132.6, 129.96, 129.93, 128.7, 128.6, 128.3, 127.9, 127.8, 127.63, 127.60, 127.59, $127.4,126.3,126.2,126.0,125.2,-0.6$.

(Z)-(2-(Anthracen-9-yl)-1,2-diphenylethenyl)dimethyl(phenyl)silane (4pa)<smiles>CC(=C(c1ccccc1)c1ccccc1)c1c2ccccc2cc2ccccc12</smiles>

$4 \mathrm{pa}$

Yellow solid. $\quad R_{\mathrm{f}}=0.29$ (dichloromethane/hexane $\left.=1 / 10\right)$. Isolated yield was $58 \%(56.7 \mathrm{mg}) . \quad{ }^{1} \mathrm{H}$ NMR (400 MHz, $\mathrm{CDCl}_{3}$ ): $\delta 8.43-8.38(\mathrm{~m}, 3 \mathrm{H}), 8.01-7.98(\mathrm{~m}, 2 \mathrm{H}), 7.51-7.46(\mathrm{~m}, 4 \mathrm{H}), 7.25-7.20(\mathrm{~m}, 2 \mathrm{H}), 7.18-7.08(\mathrm{~m}$, $6 \mathrm{H}), 6.99-6.95(\mathrm{~m}, 2 \mathrm{H}), 6.91-6.89(\mathrm{~m}, 3 \mathrm{H}), 6.83-6.81(\mathrm{~m}, 2 \mathrm{H}),-0.44(\mathrm{~s}, 6 \mathrm{H}) ;{ }^{13} \mathrm{C}\left\{{ }^{1} \mathrm{H}\right\} \mathrm{NMR}\left(101 \mathrm{MHz}, \mathrm{CDCl}_{3}\right)$ : $\delta 149.1,146.8,143.9,142.4,138.0,137.8,133.6,131.6,130.5,130.0,129.7,128.6,128.3,127.8,127.3,127.0_{4}$, 127.0 $, 126.9_{6}, 126.5,125.8,125.6,125.2,-2.0 . \quad$ FT-IR ( $\left.\mathrm{cm}^{-1}\right): 3071$ (s), 3055 (s), 2955 (s), 1596 (s), 1584 (s), 1572 (s), 1490 (s), 1480 (s), 1427 (s), 1408 (s), 1257 (s), 1245 (s), 1111 (s), 1033 (s), 943 (s), 883 (s), 865 (s), 842 (s), 830 (s), 806 (s), 781 (s), 773 (s), 760 (s), 753 (s), 733 (s), 723 (s), 703 (s). Anal. Calcd for $\mathrm{C}_{36} \mathrm{H}_{30} \mathrm{Si}$ : C, $88.11 ; \mathrm{H}, 6.16 \%$. Found: C, 87.82; H, 6.33\%.

(Z)-6-(2-(Dimethyl(phenyl)silyl)-1,2-diphenylethenyl)quinoline (4qa)<smiles>C[AsH2]C(=C(c1ccccc1)c1ccccc1)c1ccccc1</smiles>

4qa 
Yellow solid. $\quad R_{\mathrm{f}}=0.29$ (ethyl acetate/hexane $\left.=1 / 5\right)$. Isolated yield was $53 \%(46.5 \mathrm{mg}) . \quad Z: E=4: 1 . \quad{ }^{1} \mathrm{H}$ NMR (400 MHz, $\left.\mathrm{CDCl}_{3}\right)$ : (Z)-isomer (major); $\delta 8.91-8.89(\mathrm{~m}, 1 \mathrm{H}), 7.92-7.85(\mathrm{~m}, 2 \mathrm{H}), 7.53(\mathrm{~d}, J=2.0 \mathrm{~Hz}, 1 \mathrm{H})$, 7.43-7.36 (m, 3H), 7.27-7.16 (m, 6H), 7.10-7.04 (m, 3H), 7.03-6.94 (m, 5H), 0.00 (s, 6H); (E)-isomer (minor); $\delta$ 8.75-8.74 (m, 1H), 7.74-7.72 (m, 2H), 7.34-7.28 (m, 3H), 7.27-7.16 (m, 6H), 7.10-7.04 (m, 3H), 7.03-6.94 (m, 5H), 0.04 (s, 6H); ${ }^{13} \mathrm{C}\left\{{ }^{1} \mathrm{H}\right\}$ NMR (101 MHz, $\left.\mathrm{CDCl}_{3}\right): \delta 154.4,150.3,147.4,143.9_{1}, 143.85,142.6,142.1,140.4$, 136.4, 133.9, 133.7, 131.6, 129.9, 129.7, 128.9, 128.7, 128.5, 127.7, 127.6, 127.5, 126.5, 125.4, 121.3, -0.6. FT-IR (cm-1): 3067 (s), 3020 (s), 2964 (s), 2950 (s), 1594 (s), 1579 (s), 1572 (s), 1488 (s), 1439 (s), 1427 (s), 1254 (s), 1248 (s), 1233 (s), 1109 (s), 1070 (s), 1030 (s), 1002 (s), 999 (s), 945 (s), 909 (s), 899 (s), 889 (s), 847 (s), 835 (s), 819 (s), 816 (s), 809 (s), 802 (s), 795 (s), 778 (s), 771 (s), 760 (s), 751 (s), 740 (s), 721 (s), 705 (s). Anal. Calcd for $\mathrm{C}_{31} \mathrm{H}_{27} \mathrm{NSi}$ : C, 84.31; H, 6.16; N, 3.17\%. Found: C, 84.43; H, 6.15; N, 3.13\%.

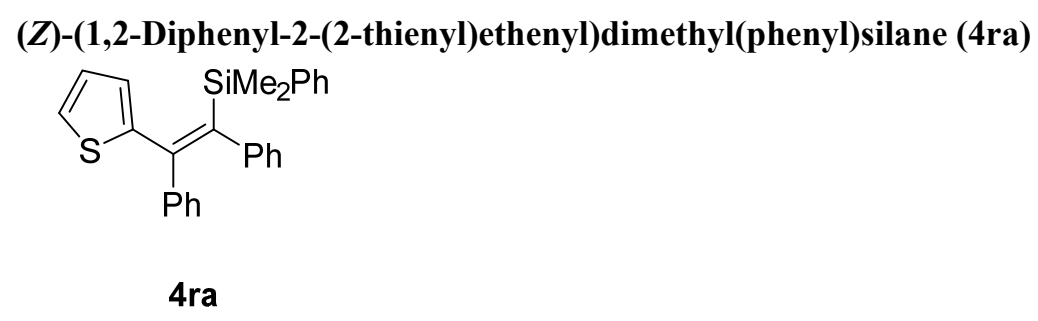

White solid. $\quad R_{\mathrm{f}}=0.38$ (dichloromethane/hexane $\left.=1 / 25\right)$. Isolated yield was $51 \%(40.1 \mathrm{mg}) . \quad{ }^{1} \mathrm{H}$ NMR (400 $\left.\mathrm{MHz}, \mathrm{CDCl}_{3}\right): \delta$ 7.38-7.22 (m, 13H), 7.14-7.11 (m, 2H), 7.05-7.03 (m, 1H), 6.68-6.66 (m, 1H), 6.26-6.25 (m, $1 \mathrm{H}),-0.07$ (s, $6 \mathrm{H}) ;{ }^{13} \mathrm{C}\left\{{ }^{1} \mathrm{H}\right\}$ NMR (101 MHz, $\left.\mathrm{CDCl}_{3}\right): \delta 145.7,145.5,143.6,142.7,141.0,139.5,134.0,130.6$, 130.1, 129.3, 129.0, 128.6, 127.9, 127.63, 127.56, 127.5, 126.5, 125.4, -1.1. FT-IR (cm $\left.{ }^{-1}\right): 3064$ (s), $3054(\mathrm{~s})$, 1560 (s), 1486 (s), 1442 (s), 1426 (s), 1256 (s), 1249 (s), 1110 (s), 1074 (s), 955 (s), 838 (s), 822 (s), 795 (s), 761 (s), 737 (s), 700 (s). Anal. Calcd for $\mathrm{C}_{26} \mathrm{H}_{24} \mathrm{SSi}$ C, $78.74 ; \mathrm{H}, 6.10 \%$. Found: C, 78.55; H, 5.90\%.

\section{(Z)-(2-(2-Furyl)-1,2-diphenylethenyl)dimethyl(phenyl)silane (4sa)}<smiles>C[Si](c1ccccc1)(c1ccccc1)C(c1ccccc1)c1ccccc1</smiles>

4sa

Colorless oil. $\quad R_{\mathrm{f}}=0.36($ dichloromethane $/$ hexane $=1 / 10)$. Isolated yield was $81 \%(61.7 \mathrm{mg}) . \quad Z: E=1: 2$. ${ }^{1} \mathrm{H}$ NMR (400 MHz, $\mathrm{CDCl}_{3}$ ): (Z)-isomer (major); $\delta$ 7.69-7.68 (m, 2H), 7.40-7.31 (m, 6H), 7.26-7.12 (m, 6H), 
6.70-6.97 (m, 2H), 6.30-6.29 (m, 1H), 5.98-5.97 (m, 1H), $0.36(\mathrm{~s}, 6 \mathrm{H})$; (E)-isomer (minor); $\delta 7.40-7.31(\mathrm{~m}, 8 \mathrm{H})$, 7.26-7.12 (m, 6H), 6.70-6.97 (m, 2H), 6.15-6.13 (m, 1H), 5.38-5.37 (m, 1H), 0.01 (s, 6H); ${ }^{13} \mathrm{C}\left\{{ }^{1} \mathrm{H}\right\} \mathrm{NMR}(101$ $\mathrm{MHz}, \mathrm{CDCl}_{3}$ ): (Z)-isomer (major); $\delta 155.3,144.1,143.3,141.8,141.6,140.85,140.79,133.5,130.5,130.0,129.2$, $127.6_{3}, 127.6_{0}, 127.4_{2}, 127.3_{8}, 125.0,111.9,111.5,0.23$; (E)-isomer (minor); $\delta$ 154.4, 144.7, 142.2, 142.0, 141.2, 140.6, 139.5, 134.0, 128.6, 128.44, 128.41, 128.2, 127.7, 127.5, 126.6, 125.7, 112.0, 111.0,-1.1. FT-IR $\left(\mathrm{cm}^{-1}\right)$ : 3067 (s), 3053 (s), 3021 (s), 2953 (s), 1804 (s), 1595 (s), 1486 (s), 1471 (s), 1440 (s), 1427 (s), 1254 (s), 1248 (s), 1113 (s), 986 (s), 842 (s), 832 (s), 813 (s), 731 (s), 699 (s). Anal. Calcd for $\mathrm{C}_{26} \mathrm{H}_{24} \mathrm{OSi}$ C, 82.06; H, 6.36\%. Found: C, $82.20 ; \mathrm{H}, 6.38 \%$.

\section{(E)-Dimethyl(phenyl)(2-phenyl-1,2-di-4-methylphenylethenyl)silane (4ab)}<smiles>Cc1ccc(C(=C(c2ccccc2)c2ccccc2)c2ccccc2)cc1</smiles>

4ab

Colorless oil. $\quad R_{\mathrm{f}}=0.55$ (dichloromethane/hexane $\left.=1 / 10\right)$. Isolated yield was $50 \%(42.1 \mathrm{mg}) . \quad{ }^{1} \mathrm{H}$ NMR (400 MHz, $\mathrm{CDCl}_{3}$ ): $\delta$ 7.40-7.38 (m, 2H), 7.34-7.24 (m, 3H), 7.24-7.22 (m, 3H), 7.19-7.16 (m, 2H), 6.97-6.95 (m, $2 \mathrm{H}), 6.89-6.84(\mathrm{~m}, 6 \mathrm{H}), 2.29(\mathrm{~s}, 3 \mathrm{H}), 2.18(\mathrm{~s}, 3 \mathrm{H}), 0.01(\mathrm{~s}, 6 \mathrm{H}) ;{ }^{13} \mathrm{C}\left\{{ }^{1} \mathrm{H}\right\} \mathrm{NMR}\left(101 \mathrm{MHz}, \mathrm{CDCl}_{3}\right): \delta 154.9$, 144.4, 141.7, 141.0, 140.6, 140.5, 135.6, 134.4, 134.0, 129.9, 129.73, 129.69, 128.5, 128.3, 128.1, 127.9, 127.5, 127.1, 21.24, 21.20,-0.6. FT-IR ( $\left.\mathrm{cm}^{-1}\right): 3067$ (s), 3049 (s), 3021 (s), 2952 (s), 2919 (s), 1576 (s), 1506 (s), 1489 (s), 1427 (s), 1255 (s), 1248 (s), 1111 (s), 1021 (s), 979 (s), 860 (s), 845 (s), 834 (s), 814 (s), 726 (s), 700 (s). Anal. Calcd for $\mathrm{C}_{30} \mathrm{H}_{30} \mathrm{Si}: \mathrm{C}, 86.07$; H, 7.22\%. Found: C, 86.15; H, 7.34\%.

\section{(E)-Dimethyl(phenyl)(2-phenyl-1,2-bis(4-(trifluoromethyl)phenyl)ethenyl)silane (4ac)}<smiles>CC(C)(C)c1ccc(C(=C(c2ccccc2)c2ccccc2)c2ccc(C(F)(F)F)cc2)cc1</smiles> 
Colorless oil. $\quad R_{\mathrm{f}}=0.54$ (dichloromethane/hexane $\left.=1 / 10\right)$. Isolated yield was $46 \%(48.1 \mathrm{mg}) . \quad{ }^{1} \mathrm{H}$ NMR (400 MHz, $\left.\mathrm{CDCl}_{3}\right): \delta$ 7.38-7.32 (m, 3H), 7.31-7.24 (m, 9H), 7.18-7.16 (m, 2H), 7.04-6.99 (m, 4H), $0.02(\mathrm{~s}, 6 \mathrm{H})$; ${ }^{13} \mathrm{C}\left\{{ }^{1} \mathrm{H}\right\}$ NMR (101 MHz, $\left.\mathrm{CDCl}_{3}\right): \delta 154.4,147.4,146.4,143.5,142.8,139.0,133.9,129.8,129.7,129.5,129.1$, $128.5\left(\mathrm{q}, J_{\mathrm{C}-\mathrm{F}}=31.4 \mathrm{~Hz}\right), 128.3,127.9,127.8,127.7\left(\mathrm{q}, J_{\mathrm{C}-\mathrm{F}}=32.5 \mathrm{~Hz}\right), 124.7_{0}\left(\mathrm{q}, J_{\mathrm{C}-\mathrm{F}}=4.0 \mathrm{~Hz}\right), 124.6_{8}\left(\mathrm{q}, J_{\mathrm{C}-\mathrm{F}}\right.$ $=3.7 \mathrm{~Hz}), 124.4\left(\mathrm{q}, J_{\mathrm{C}-\mathrm{F}}=273.1 \mathrm{~Hz}\right), 124.1\left(\mathrm{q}, J_{\mathrm{C}-\mathrm{F}}=273.4 \mathrm{~Hz}\right),-1.0 ;{ }^{19} \mathrm{~F}\left\{{ }^{1} \mathrm{H}\right\} \mathrm{NMR}\left(376 \mathrm{MHz}, \mathrm{CDCl}_{3}, \mathrm{rt}\right): \delta-$ 62.6, -62.9. FT-IR ( $\left.\mathrm{cm}^{-1}\right)$ : 3065 (s), 3049 (s), 2957 (s), 1598 (s), 1383 (s), 1326 (s), 1165 (s), 1123 (s), 1106 (s), 831 (s), 815 (s), 750 (s). Anal. Calcd for $\mathrm{C}_{30} \mathrm{H}_{24} \mathrm{~F}_{6} \mathrm{Si}$ : C, 68.43; H, 4.59\%. Found: C, 68.43; H, 4.45\%.

\section{(E)-Dimethyl(phenyl)(2-phenyl-1,2-di(thie-2-yl)ethenyl)silane (4ad)}<smiles></smiles>

Colorless oil. $\quad R_{\mathrm{f}}=0.45$ (dichloromethane/hexane $\left.=1 / 10\right)$. Isolated yield was $44 \%(35.6 \mathrm{mg}) . \quad Z: E=4: 1$. ${ }^{1} \mathrm{H}$ NMR (400 MHz, $\mathrm{CDCl}_{3}$ ): (Z)-isomer (major); $\delta$ 7.35-7.11 (m, 12H), 7.05-7.03 (m, 1H), 6.73-6.68 (m, 2H), 6.37-6.36 (m, 1H), -0.05 (s, 6H); (E)-isomer (minor); $\delta 7.53-7.50(\mathrm{~m}, 2 \mathrm{H}), 7.35-7.11(\mathrm{~m}, 9 \mathrm{H}), 7.01-6.99(\mathrm{~m}, 1 \mathrm{H})$, 6.91-6.90 (m, 1H), 6.87-6.85 (m, 1H), 6.77-6.75 (m, 1H), 6.51-6.50 (m, 1H), 0.17 (s, 6H); ${ }^{13} \mathrm{C}\left\{{ }^{1} \mathrm{H}\right\}$ NMR (101 $\left.\mathrm{MHz}, \mathrm{CDCl}_{3}\right): \delta 150.1,144.8,144.4,142.4,139.4,133.9_{9}, 133.97,130.9,130.4,128.7,128.6,127.9,127.8,127.7$, 127.6, 126.9, 126.0, 125.5, -1.3. FT-IR ( $\left.\mathrm{cm}^{-1}\right): 3067$ (s), 3050 (s), 2953 (s), 1558 (s), 1487 (s), 1427 (s), 1254 (s), 1248 (s), 1112 (s), 848 (s), 836 (s), 828 (s), 819 (s), 793 (s), 733 (s), 697 (s). Anal. Calcd for $\mathrm{C}_{24} \mathrm{H}_{22} \mathrm{~S}_{2} \mathrm{Si}$ : C, 71.59; H, 5.51\%. Found: C, 71.50; H, 5.47\%.

\section{(E)-(1,2-Di(naphthalen-2-yl)-2-phenylethenyl)dimethyl(phenyl)silane (4ae)}<smiles>CCC(C(=C1C=Cc2ccccc21)c1ccccc1)=C(c1ccc2ccccc2c1)c1ccc2ccccc2c1</smiles>

$4 a e$ 
Yellow oil. $\quad R_{\mathrm{f}}=0.48($ dichloromethane $/$ hexane $=1 / 10)$. Isolated yield was 34\% $(33.1 \mathrm{mg}) . \quad Z: E=10: 1$. ${ }^{1} \mathrm{H}$ NMR (400 MHz, $\mathrm{CDCl}_{3}$ ): (Z)-isomer (major); $\delta$ 7.75-7.69 (m, 1H), 7.68-7.64 (m, 1H), 7.61-7.51 (m, 3H), 7.49-7.29 (m, 17H), 7.26-7.22 (m, 1H), 7.19-7.16 (m, 1H), $0.10(\mathrm{~s}, 6 \mathrm{H}) ;(E)$-isomer (minor); $\delta$ 7.87-7.84 (m, $1 \mathrm{H})$, 7.81-7.78 (m, 1H), 7.75-7.69 (m, 3H), 7.68-7.64 (m, 1H), 7.61-7.51 (m, 3H), 7.49-7.29 (m, 8H), 7.26-7.22 (m, 2H), 7.19-7.16 (m, 1H), 7.07-7.03 (m, 1H), 7.00-6.89 (m, 3H), $0.05(\mathrm{~s}) ;{ }^{13} \mathrm{C}\left\{{ }^{1} \mathrm{H}\right\}$ NMR (101 MHz, $\left.\mathrm{CDCl}_{3}\right): \delta$ $155.3,144.1,142.9,141.6,140.9,140.2,134.1,133.2,132.9,131.8,131.5,130.0,129.2,128.9,128.7,128.1_{0}$, $128.0_{5}, 127.9_{9}, 127.9_{7}, 127.8,127.7,127.6,127.5,127.4,127.0,126.8,125.69,125.67,125.64,125.1,-0.7$. FTIR ( $\left.\mathrm{cm}^{-1}\right)$ : 3065 (s), 3020 (s), 2052 (s), 2921 (s), 2898 (s), 1616 (s), 1583 (s), 1503 (s), 1423 (s), 1400 (s), 1230 (s), 978 (s), 880 (s). Anal. Calcd for $\mathrm{C}_{36} \mathrm{H}_{30} \mathrm{Si}$ : C, 88.11; H, 6.16\%. Found: C, 87.98; H, 6.45\%.

\section{(1,1-Diphenylprop-1-en-2-yl)dimethyl(phenyl)silane (4af)}<smiles>CC(=C(c1ccccc1)c1ccccc1)c1ccccc1</smiles>

Colorless oil. $\quad R_{\mathrm{f}}=0.56$ (dichloromethane/hexane $\left.=1 / 10\right)$. Isolated yield was $38 \%(25.0 \mathrm{mg})$. Ratio of regioisomers $=10 / 1 . \quad{ }^{1} \mathrm{H}$ NMR $\left(400 \mathrm{MHz}, \mathrm{CDCl}_{3}\right)$ : major regioisomer; $\delta$ 7.54-7.50 $(\mathrm{m}, 2 \mathrm{H}), 7.38-7.34(\mathrm{~m}, 3 \mathrm{H})$, 7.33-7.28 (m, 2H), 7.25-7.14 (m, 6H), 7.12-7.08 (m, 2H), $1.86(\mathrm{~s}, 3 \mathrm{H}), 0.05(\mathrm{~s}, 6 \mathrm{H})$; minor regioisomer; $\delta$ 7.54$7.50(\mathrm{~m}, 1 \mathrm{H}), 7.33-7.28(\mathrm{~m}, 1 \mathrm{H}), 7.25-7.14(\mathrm{~m}, 10 \mathrm{H}), 7.05-6.99(\mathrm{~m}, 3 \mathrm{H}), 1.83(\mathrm{~s}, 3 \mathrm{H}),-0.11(\mathrm{~s}, 6 \mathrm{H}) ;{ }^{13} \mathrm{C}\left\{{ }^{1} \mathrm{H}\right\}$ NMR (101 MHz, $\left.\mathrm{CDCl}_{3}\right): \delta$ 154.1, 145.1, 143.5, 140.4, 133.9, 133.8, 129.8, 129.3, 128.7, 128.1, 127.9, 127.8, 127.0, 126.6, 21.6, -1.4. FT-IR (cm-1): 3067 (s), 2954 (s), 2912 (s), 1497 (s), 1421 (s), 1402 (s), 1221 (s), 1088 (s), 821 (s), 800 (s), 796 (s). Anal. Calcd for $\mathrm{C}_{23} \mathrm{H}_{24} \mathrm{Si}$ : C, 84.09; H, 7.36\%. Found: C, 84.15; H, 7.52\%.

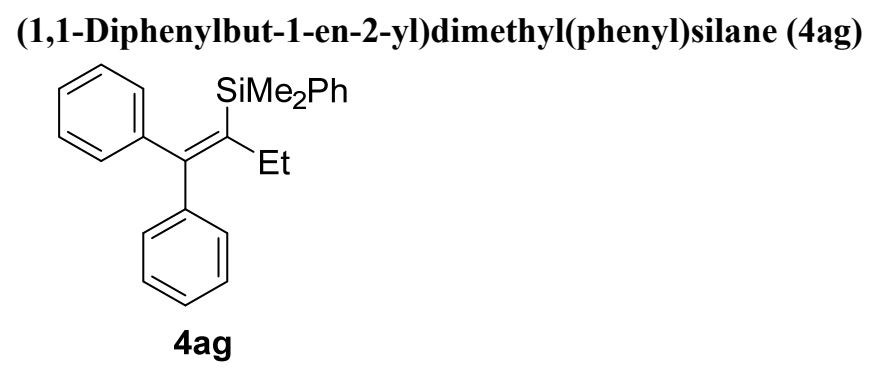


Colorless oil. $\quad R_{\mathrm{f}}=0.54$ (dichloromethane/hexane $\left.=1 / 10\right)$. Isolated yield was 43\% (29.3 mg). ${ }^{1} \mathrm{H} \mathrm{NMR}$ (400 MHz, $\left.\mathrm{CDCl}_{3}\right): \delta$ 7.49-7.47 (m, 2H), 7.34-7.29 (m, 5H), 7.21-7.09 (m, 8H), 2.23 (q, J=7.4 Hz, $\left.2 \mathrm{H}\right), 0.87(\mathrm{t}$, $J=7.4 \mathrm{~Hz}, 3 \mathrm{H}), 0.12(\mathrm{~s}, 6 \mathrm{H}) ;{ }^{13} \mathrm{C}\left\{{ }^{1} \mathrm{H}\right\} \mathrm{NMR}\left(101 \mathrm{MHz}, \mathrm{CDCl}_{3}\right): \delta 154.9,144.9,144.1,140.8,140.6,133.9$,

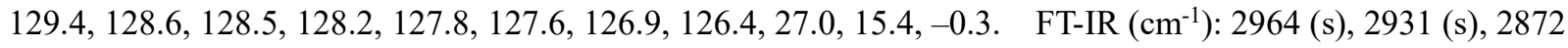
(s), 1497 (s), 1420 (s), 1412 (s), 1320 (s), 1090 (s), 839 (s), 828 (s), 808 (s), 722 (s), 700 (s). Anal. Calcd for $\mathrm{C}_{24} \mathrm{H}_{26} \mathrm{Si}: \mathrm{C}, 84.15$; H, 7.65\%. Found: C, 83.88; H, 7.59\%.

\section{Tert-butyl((2-(dimethyl(phenyl)silyl)-3,3-diphenylallyl)oxy)dimethylsilane (4ah)}<smiles>CC(C)COCC(=C(c1ccccc1)c1ccccc1)c1ccccc1</smiles>

Colorless oil. $\quad R_{\mathrm{f}}=0.45$ (dichloromethane/hexane $\left.=1 / 10\right)$. Isolated yield was 53\% (48.6 mg). Ratio of regioisomers $=10 / 1 . \quad{ }^{1} \mathrm{H} \mathrm{NMR}\left(400 \mathrm{MHz}, \mathrm{CDCl}_{3}\right)$ : major regioisomer; $\delta 7.51-7.48(\mathrm{~m}, 2 \mathrm{H}), 7.30-7.21(\mathrm{~m}, 6 \mathrm{H})$, 7.19-7.09 (m, 5H), 7.02-6.99 (m, 2H), $4.27(\mathrm{~s}, 2 \mathrm{H}), 0.85(\mathrm{~s}, 9 \mathrm{H}), 0.15(\mathrm{~s}, 6 \mathrm{H}),-0.11(\mathrm{~s}, 6 \mathrm{H})$; minor regioisomer; $\delta 7.62-7.60(\mathrm{~m}, 2 \mathrm{H}), 7.37-7.31(\mathrm{~m}, 6 \mathrm{H}), 7.30-7.21(\mathrm{~m}, 5 \mathrm{H}), 7.19-7.09(\mathrm{~m}, 2 \mathrm{H}), 4.53(\mathrm{~s}, 2 \mathrm{H}), 0.85(\mathrm{~s}, 9 \mathrm{H}), 0.51(\mathrm{~s}$, $6 \mathrm{H}),-0.05(\mathrm{~s}, 6 \mathrm{H}) ;{ }^{13} \mathrm{C}\left\{{ }^{1} \mathrm{H}\right\} \mathrm{NMR}\left(101 \mathrm{MHz}, \mathrm{CDCl}_{3}\right): \delta 155.9,144.2,142.7,141.0,138.6,134.2,129.7,129.4$, 128.3 127.8, 127.7, 127.4, 127.24, 127.19, 64.9, 26.1, 18.5, -0.5, -5.3. FT-IR (cm $\left.{ }^{-1}\right): 2955$ (s), 2928 (s), 2855 (s), 1498 (s), 1450 (s), 1410 (s), 1402 (s), 1252 (s), 1080 (s), 1055 (s), 835 (s), 821 (s), 701 (s). Anal. Calcd for $\mathrm{C}_{29} \mathrm{H}_{38} \mathrm{OSi}_{2}$ : C, 75.92; H, 8.35\%. Found: C, 75.97; H, 8.45\%.

\section{(Z)-Methyl(2-(naphthalen-2-yl)-1,2-diphenylethenyl)diphenylsilane (4oa-SiMePh $)$}<smiles></smiles>

\section{4oa-SiMePh 2}

White solid. $\quad R_{\mathrm{f}}=0.33$ (dichloromethane/hexane $\left.=1 / 10\right) . \quad$ Isolated yield was 55\% (54.9 mg). $\quad{ }^{1} \mathrm{H} \mathrm{NMR}(400$ $\left.\mathrm{MHz}, \mathrm{CDCl}_{3}\right): \delta$ 7.72-7.69 (m, 1H), 7.60-7.59 (m, 1H), $7.50(\mathrm{~d}, J=8.4 \mathrm{~Hz}, 1 \mathrm{H}), 7.44-7.40(\mathrm{~m}, 1 \mathrm{H}), 7.38-7.34$ $(\mathrm{m}, 6 \mathrm{H}), 7.27-7.23(\mathrm{~m}, 2 \mathrm{H}), 7.20-7.15(\mathrm{~m}, 4 \mathrm{H}), 7.10-7.08(\mathrm{~m}, 1 \mathrm{H}), 7.02-6.95(\mathrm{~m}, 8 \mathrm{H}), 6.92-6.90(\mathrm{~m}, 2 \mathrm{H}), 0.15$ 
(m, 3H); ${ }^{13} \mathrm{C}\left\{{ }^{1} \mathrm{H}\right\}$ NMR (101 MHz, $\left.\mathrm{CDCl}_{3}\right): \delta 156.8,143.8,143.1,141.3,141.1,138.0,135.0,132.7,132.6$, 130.5, 130.2, 129.0, 128.7, 128.3, 127.8, 127.6, 127.52, 127.49, 127.40, 127.37, 126.5, 125.97, 125.92, 125.1,-1.7. FT-IR ( $\left.\mathrm{cm}^{-1}\right)$ : 3025 (s), 3014 (s), 3008 (s), 1573 (s), 1538 (s), 1461 (s), 1426 (s), 1258 (s), 1115 (s), 1105 (s), 991 (s), 973 (s), 901 (s), 861 (s), 829 (s), 794 (s), 785 (s), 749 (s), 740 (s). Anal. Calcd for $\mathrm{C}_{37} \mathrm{H}_{30} \mathrm{Si}: \mathrm{C}, 88.40$; H, $6.02 \%$. Found: C, $88.27 ; \mathrm{H}, 5.81 \%$.

\section{(Z)-Triethyl(2-(naphthalen-2-yl)-1,2-diphenylethenyl)silane (40a-SiEt 3 )}<smiles>CC[Si](=C(c1ccccc1)c1ccccc1)c1ccc2ccccc2c1</smiles>

4oa-SiEt 3

Colorless oil. $\quad R_{\mathrm{f}}=0.41$ (dichloromethane/hexane $\left.=1 / 10\right)$. Isolated yield was $41 \%(34.5 \mathrm{mg}) . \quad{ }^{1} \mathrm{H}$ NMR (400 MHz, $\left.\mathrm{CDCl}_{3}\right): \delta$ 7.95-7.91 (m, 2H), 7.88-7.86 (m, 1H), 7.82-7.80 (d, $\left.J=8.4 \mathrm{~Hz}, 1 \mathrm{H}\right), 7.57-7.49(\mathrm{~m}, 2 \mathrm{H})$, 7.44-7.41 (m, 1H), 7.20-7.15 (m, 2H), 7.08-6.95 (m, 8H), 0.87 (t, $J=8.0 \mathrm{~Hz}, 9 \mathrm{H}), 0.29$ (q, $J=8.0 \mathrm{~Hz}, 6 \mathrm{H})$; ${ }^{13} \mathrm{C}\left\{{ }^{1} \mathrm{H}\right\} \mathrm{NMR}\left(101 \mathrm{MHz}, \mathrm{CDCl}_{3}\right): \delta$ 155.1, 144.5, 143.4, 142.4, 141.6, 133.0, 132.6, 129.6, 129.4, 128.1, 127.9, 127.70, 127.65, 127.6, 127.5, 127.4, 126.3, 126.1, 126.0, 124.9, 7.8, 4.6. FT-IR ( $\left.\mathrm{cm}^{-1}\right): 2952$ (s), 2931 (s), 2872 (s), 1595 (s), 1486 (s), 1420 (s), 1237 (s), 1000 (s), 859 (s), 828 (s), 732 (s), 726 (s), 697 (s). Anal. Calcd for $\mathrm{C}_{30} \mathrm{H}_{32} \mathrm{Si}$ : C, 85.66; H, 7.67\%. Found: C, 85.36; H, 7.69\%.

\section{(Z)-Tert-butyldimethyl(2-(naphthalen-2-yl)-1,2-diphenylethenyl)silane (4oa-SiMe $\left.{ }_{2}{ }^{t} \mathrm{Bu}\right)$}<smiles>CC(C)(C)C(=C(c1ccccc1)c1ccccc1)c1ccccc1</smiles>

\section{4oa-SiMe ${ }_{2}{ }^{t} \mathrm{Bu}$}

White solid. $\quad R_{\mathrm{f}}=0.35$ (dichloromethane/hexane $\left.=1 / 10\right) . \quad$ Isolated yield was $36 \%(30.1 \mathrm{mg}) . \quad{ }^{1} \mathrm{H}$ NMR $(400$ $\left.\mathrm{MHz}, \mathrm{CDCl}_{3}\right): \delta$ 7.95-7.94 (m, 1H), 7.91-7.89 (m, 1H), 7.86-7.84 (m, 1H), 7.79-7.76 (m, 1H), 7.55-7.48 (m, 2H), 7.40-7.37 (m, 1H), 7.16-7.09 (m, 4H), 7.05-6.91 (m, 6H), 0.80 (s, 9H), $-0.24(\mathrm{~s}, 6 \mathrm{H}) ;{ }^{13} \mathrm{C}\left\{{ }^{1} \mathrm{H}\right\} \mathrm{NMR}(101 \mathrm{MHz}$, $\left.\mathrm{CDCl}_{3}\right): \delta 155.9,145.0,144.0,142.7,142.1,133.0,132.5,130.5,129.8,128.1_{1}, 128.08,128.0,127.9,127.6$, 127.39, 127.37, 126.3, 126.0 (x 2), 125.2, 28.0, 18.5, -2.4. FT-IR ( $\left.\mathrm{cm}^{-1}\right): 2953$ (s), 2928 (s), 2855 (s), 1554 (s), 
$1548(\mathrm{~s}), 1487$ (s), 1472 (s), 1462 (s), 1255 (s), 1248 (s), 1053 (s), 1010 (s), 943 (s), 882 (s), 863 (s), 840 (s), 829 (s), 819 (s), 800 (s). Anal. Calcd for $\mathrm{C}_{30} \mathrm{H}_{32} \mathrm{Si}: \mathrm{C}, 85.66 ; \mathrm{H}, 7.67 \%$. Found: C, 85.62; H, 7.64\%. 


\section{Substrate Scope}

The products shown below were not obtained at all.<smiles></smiles><smiles>C[As](C(=C(c1ccccc1)c1ccccc1)c1ccccc1)c1ccccc1</smiles><smiles>CC(=O)Oc1ccccc1/C(=C(\[SiH2]c1ccccc1)c1ccccc1)c1ccccc1</smiles><smiles>Clc1ccc(C(=C(c2ccccc2)c2ccccc2)c2ccccc2)cc1</smiles><smiles>C[SiH](C(=C(c1ccccc1)c1ccccc1)c1cccnc1)c1cccnc1</smiles><smiles>Clc1cccc(C(=C(c2ccccc2)c2cccc(Cl)c2)c2ccccc2)c1</smiles><smiles>Clc1ccc(C(=C(c2ccc(Cl)cc2)C([AsH2])c2ccccc2)c2ccccc2)cc1</smiles><smiles>c1ccc([AsH2]C(=C(c2ccccc2)c2ccccc2)C2CC2)cc1</smiles><smiles>C[SiH2]C(CNC(=O)c1ccccc1)=C(c1ccccc1)c1ccccc1</smiles><smiles>CC[SiH2]C(C(=Cc1ccccc1)CC)=C(c1ccccc1)c1ccccc1</smiles><smiles>CC(C(Br)=C(c1ccccc1)c1ccccc1)c1ccccc1</smiles><smiles>COC(=O)C(=C(c1ccccc1)c1ccccc1)C(OC)OC</smiles>

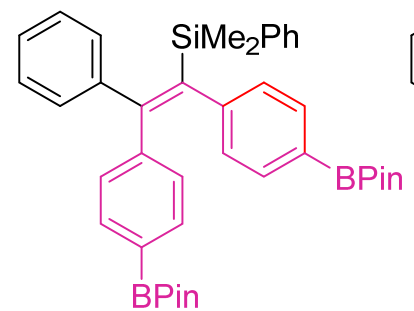<smiles></smiles><smiles>CC(C=C(c1ccccc1)c1ccccc1)c1ccccc1</smiles><smiles>C(#Cc1ccccc1)C=C([SiH2]c1ccccc1)C(c1ccccc1)c1ccccc1</smiles> 
4.3 The reaction in $1 \mathrm{mmol}$ scale.

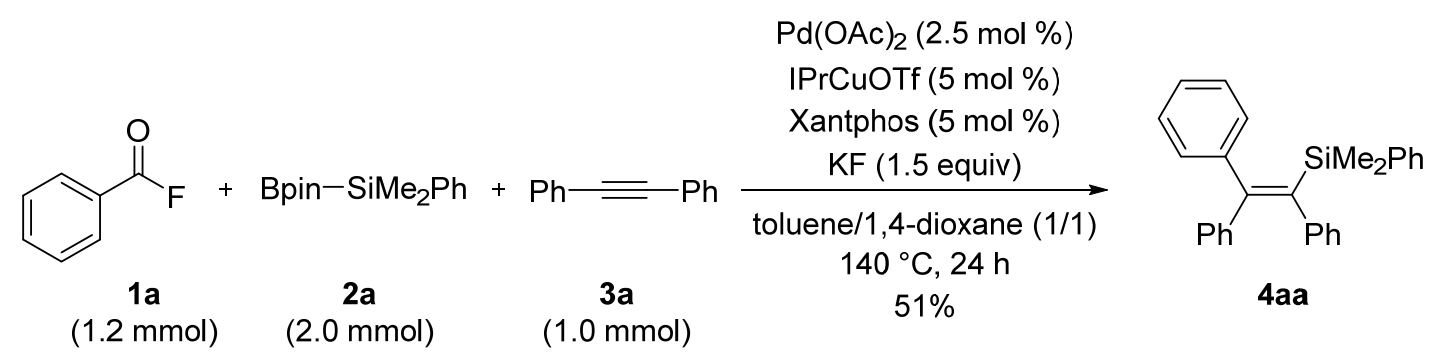

An oven-dried $50 \mathrm{~mL}$ of Schlenk tube containing a magnetic stirring bar was charged with $\operatorname{Pd}(\mathrm{OAc})_{2}(5.5 \mathrm{mg}$, $0.025 \mathrm{mmol}, 2.5 \mathrm{~mol} \%$ ), Xantphos (29 mg, $0.05 \mathrm{mmol}, 5 \mathrm{~mol} \%$ ), IPrCuOTf (30 mg, $0.05 \mathrm{mmol}, 5 \mathrm{~mol} \%$ ), KF ( $87 \mathrm{mg}, 0.3 \mathrm{mmol}, 1.5$ equiv), toluene $(2.5 \mathrm{~mL})$, and 1,4-dioxane $(2.5 \mathrm{~mL})$ under argon, which was stirred for 30 seconds at room temperature. Then, benzoyl fluoride 1a (149 mg, $1.2 \mathrm{mmol})$, dimethylphenylsilylboronic acid pinacol ester 2a (526 mg, $2 \mathrm{mmol})$, and diphenylacetylene 3a (178.2 mg, $1.0 \mathrm{mmol})$ were added. The mixture was heated at $140{ }^{\circ} \mathrm{C}$ in a heating block with stirring for $24 \mathrm{~h}$. After being at room temperature, the mixture was quenched with saturated $\mathrm{NH}_{4} \mathrm{Cl}$ and then aqueous solution was extracted with diethyl ether. The combined organic phase was dried over anhydrous $\mathrm{MgSO}_{4}$, and evaporated under vacuum to remove the volatiles. The residue was purified by column chromatography (EtOAc or $\mathrm{CH}_{2} \mathrm{Cl}_{2}$ /hexane) on silica gel to afford the desired products 4aa. White solid. $R_{\mathrm{f}}=0.18$ (hexane). Isolated yield was $51 \%(201 \mathrm{mg})$. 


\section{Mechanistic Studies}

\subsection{Preparation of 6 aa an authentic sample (for Scheme $2 \mathrm{~b}$ ). ${ }^{8}$}

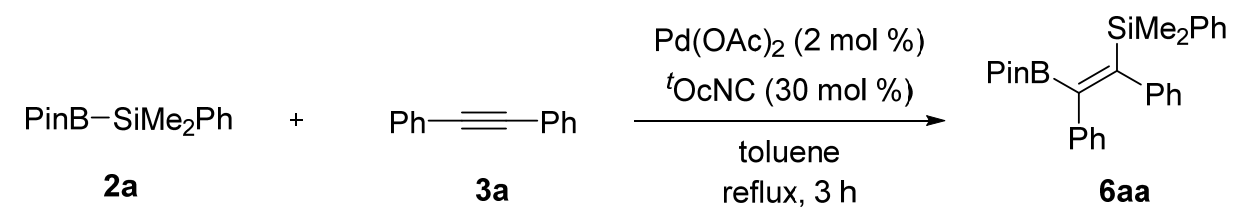

An oven-dried $20 \mathrm{~mL}$ of Schlenk tube containing a magnetic stirring bar was charged with $\mathrm{Pd}(\mathrm{OAc})_{2}(4.5 \mathrm{mg}$, $0.02 \mathrm{mmol}, 2 \mathrm{~mol} \%$ ) and 1,1,3,3-tetramethylbutyl isocyanide (42 mg, $0.30 \mathrm{mmol}, 30 \mathrm{~mol} \%$ ) under argon, which was stirred at room temperature until a deep red color was observed. Then, toluene $(0.5 \mathrm{~mL})$, dimethylphenylsilylboronic acid pinacol ester (2a) $(1 \mathrm{mmol})$ and alkyne 3a $(2.0 \mathrm{mmol})$ were added. The mixture was heated at $120^{\circ} \mathrm{C}$ in a heating block with stirring for $3 \mathrm{~h}$. After being at room temperature, the mixture was evaporated under vacuum to remove the volatiles. The residue was purified by column chromatography $\left(\right.$ EtOAc /hexane $=20 / 1, R_{\mathrm{f}}=0.36$ ) on silica gel to afford colorless solid 6 in $68 \%$ isolated yield (301.1 mg). $\quad{ }^{1} \mathrm{H}$ NMR (400 MHz, $\left.\mathrm{CDCl}_{3}\right): \delta$ 7.66-7.63 (m, 2H), 7.36-7.33 (m, 3H), 7.04-6.91 (m, 8H), 6.72$6.70(\mathrm{~m}, 2 \mathrm{H}), 1.05(\mathrm{~m}, 12 \mathrm{H}), 0.34(\mathrm{~s}, 6 \mathrm{H})$.

\subsection{Reaction with Aliphatic Alkynes 3i (Scheme 2d).}

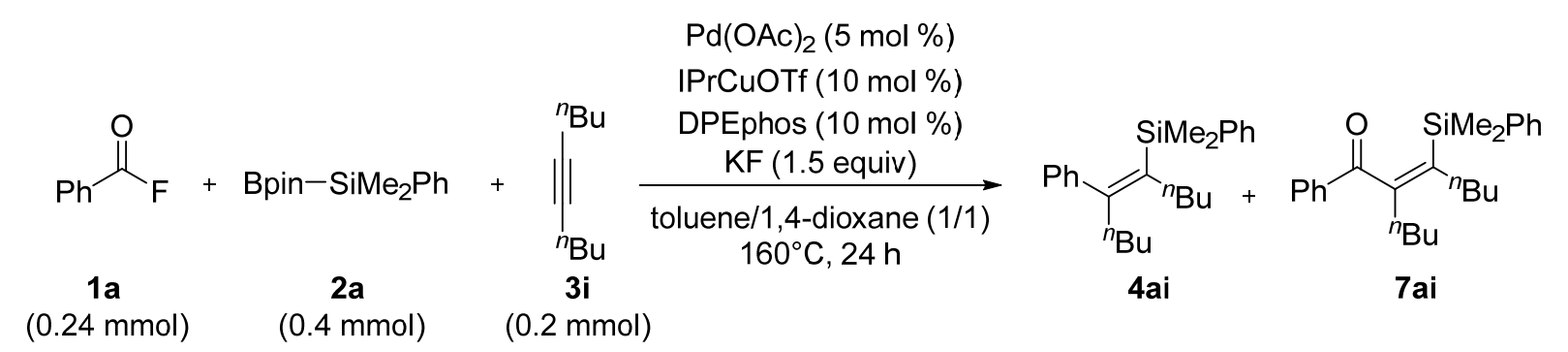

An oven-dried $20 \mathrm{~mL}$ of Schlenk tube containing a magnetic stirring bar was charged with $\mathrm{Pd}(\mathrm{OAc})_{2}(2.2 \mathrm{mg}$, $0.01 \mathrm{mmol}, 5 \mathrm{~mol} \%$ ), DPEphos (10.8 mg, $0.02 \mathrm{mmol}, 10 \mathrm{~mol} \%)$, IPrCuOTf(12 mg, $0.02 \mathrm{mmol}, 10 \mathrm{~mol} \%), \mathrm{KF}$ (17.4 mg, $0.3 \mathrm{mmol}, 1.5$ equiv), toluene $(0.5 \mathrm{~mL})$, and 1,4-dioxane $(0.5 \mathrm{~mL})$ under argon, which was stirred for 30 seconds at room temperature. Then, benzoyl fluorides $\mathbf{1 a}(0.24 \mathrm{mmol})$, dimethylphenylsilylboronic acid pinacol ester (2a) $(0.4 \mathrm{mmol})$, and 5-decyne (3i) $(0.2 \mathrm{mmol})$ were added. The mixture was heated at $160^{\circ} \mathrm{C}$ in a heating block with stirring for $24 \mathrm{~h}$. After cooling to room temperature, the mixture was quenched with saturated $\mathrm{NH}_{4} \mathrm{Cl}$ and then aqueous solution was extracted with diethyl ether. The combined organic phase was dried over anhydrous $\mathrm{MgSO}_{4}$, and evaporated under vacuum to remove the volatiles. The residue was purified 
by column chromatography $\left(\mathrm{CH}_{2} \mathrm{Cl}_{2} /\right.$ hexane $\left.=1 / 1, R_{\mathrm{f}}=0.5\right)$ on silica gel to afford 7 ai in $30 \%$ isolated yield $(22.7$ $\mathrm{mg}$ ) as a colorless oil. $\quad{ }^{1} \mathrm{H}$ NMR $\left(400 \mathrm{MHz}, \mathrm{CDCl}_{3}\right): \delta$ 7.80-7.77 (m, 2H), 7.53-7.49 (m, 1H), 7.44-7.36 (m, 4H), 7.24-7.20 (m, 3H), 2.40-2.38 (m, 2H), 2.24-2.20 (m, 2H), 1.34-1.23 (m, 8H), 0.88-0.81 (m, 6H), $0.23(\mathrm{~s}, 6 \mathrm{H})$; ${ }^{13} \mathrm{C}\left\{{ }^{1} \mathrm{H}\right\}$ NMR (101 MHz, $\left.\mathrm{CDCl}_{3}\right): \delta 201.0 .152 .3,139.7,138.5,137.0,134.4,132.9,129.6,128.8,128.4,127.5$, 32.5, 31.54, 31.46, 30.8, 23.4, 22.9, 14.0 (x 2), -1.0. FT-IR ( $\left.\mathrm{cm}^{-1}\right): 3013$ (s), 2960 (s), 2931 (s), 2873 (s), 2862 (s), 1663 (s), 1259 (s), 1250 (s), 1216 (s), 762 (s), 757 (s). Anal. Calcd for $\mathrm{C}_{25} \mathrm{H}_{34} \mathrm{OSi}$ : C, 79.31; H, 9.05\%. Found: C, 79.27; H, 9.30\%. 


\section{Copies of ${ }^{1} \mathrm{H},{ }^{13} \mathrm{C}\left\{{ }^{1} \mathrm{H}\right\}$, and ${ }^{19} \mathrm{~F}\left\{{ }^{1} \mathrm{H}\right\}$ NMR Charts for the Products}

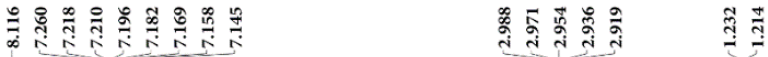
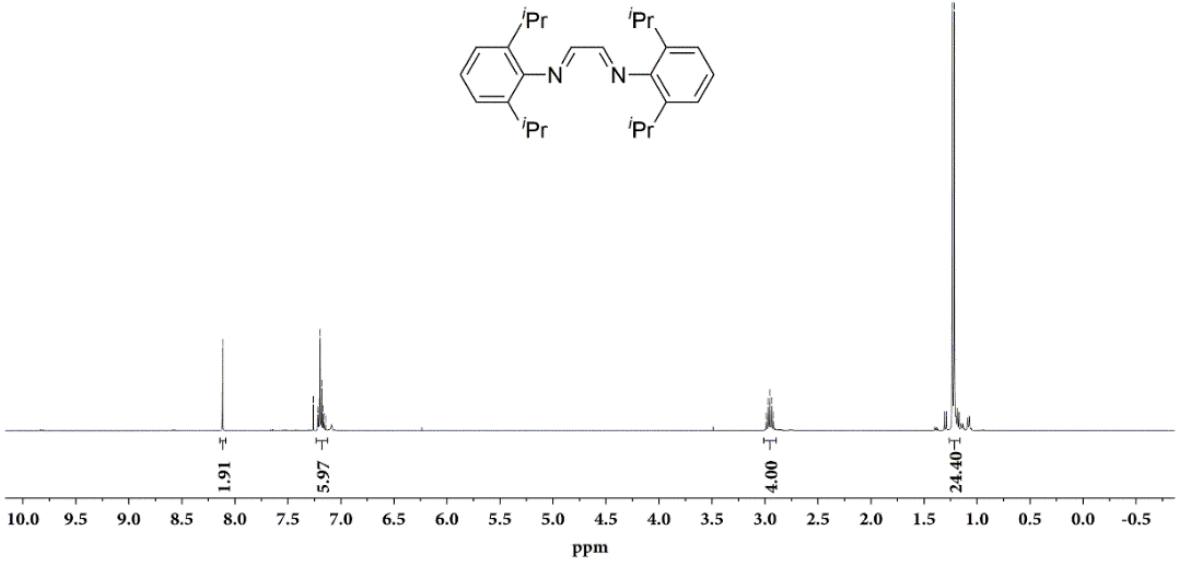

${ }^{1} \mathrm{H}$ NMR (400 MHz) spectra of N,N'-1,4-bis(2,6-diisopropylphenyl)-1,4-diazabutadiene (rt, $\mathrm{CDCl}_{3}$ ).
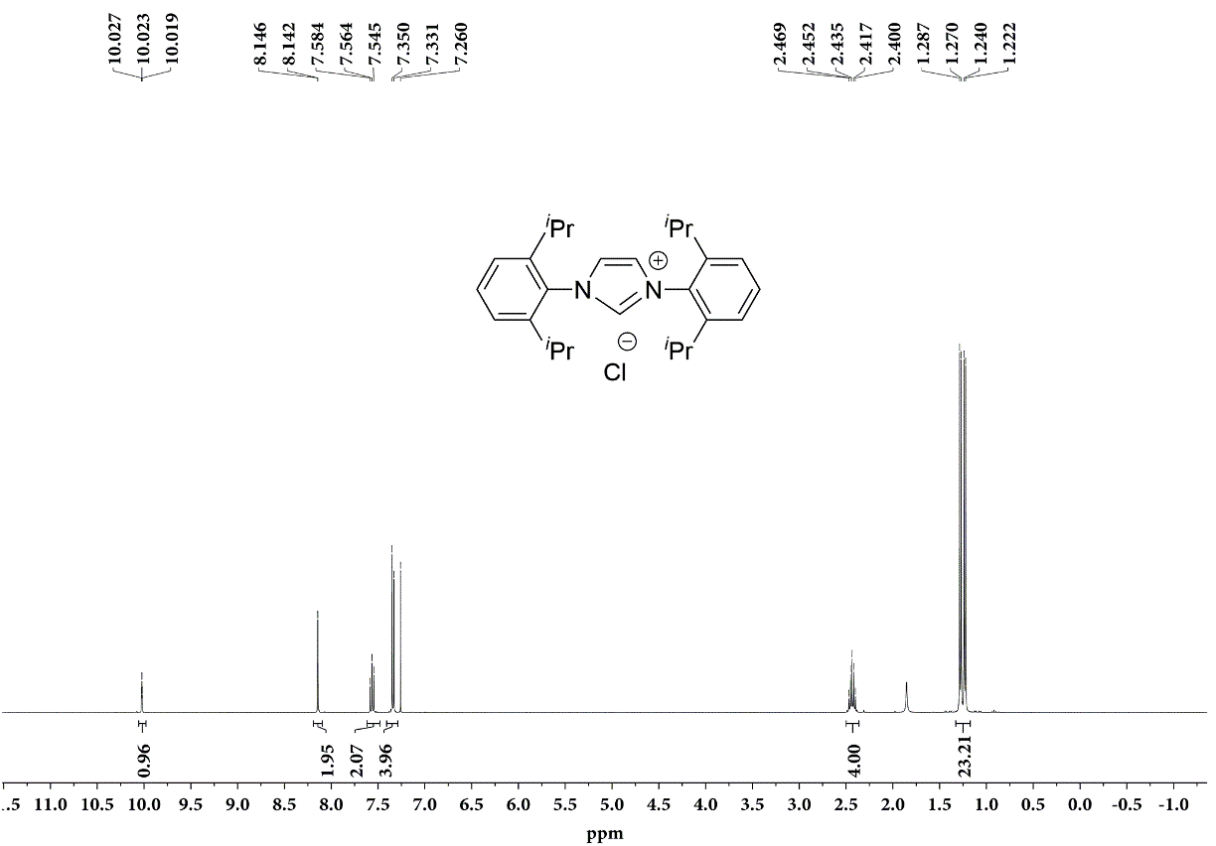

${ }^{1} \mathrm{H}$ NMR (400 MHz) spectra of (N,N'-1,3-bis(2,6-diisopropylphenyl)imidazolium chloride) (rt, $\mathrm{CDCl}_{3}$ ). 

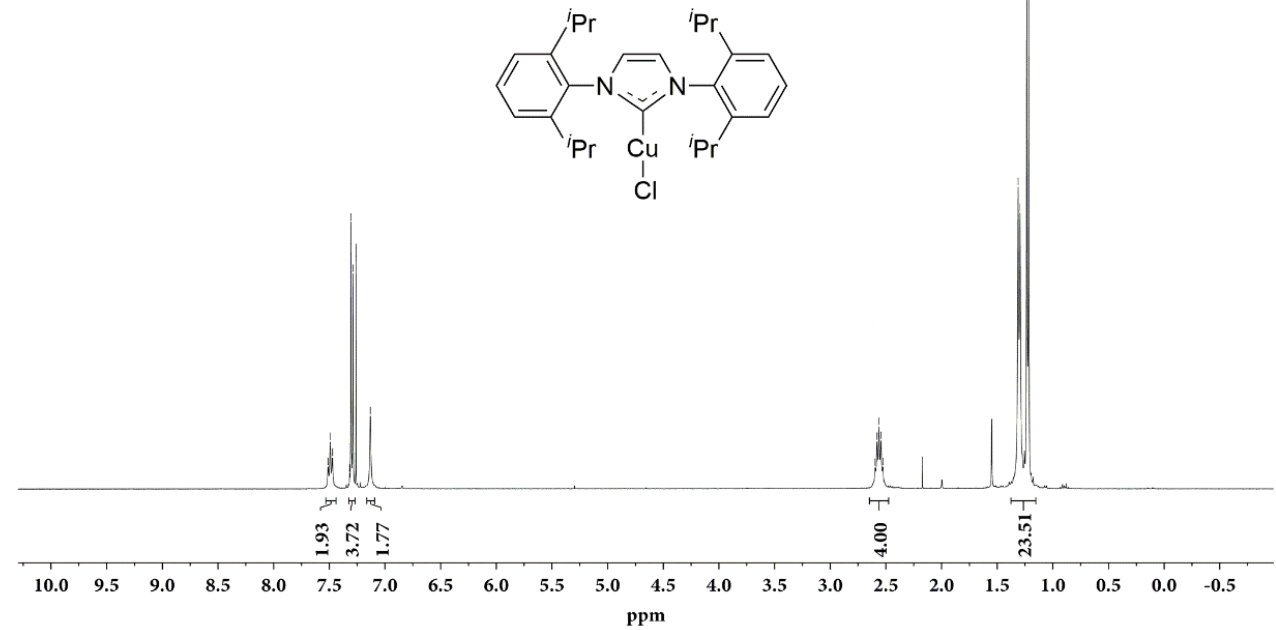

${ }^{1} \mathrm{H}$ NMR (400 MHz) spectra of [1,3-bis(2,6-di-iso-propylphenyl)imidazol-2-ylidene] copper (I) chloride (rt, $\left.\mathrm{CDCl}_{3}\right)$.

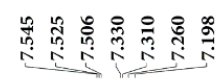

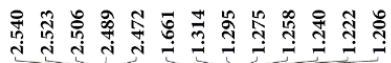
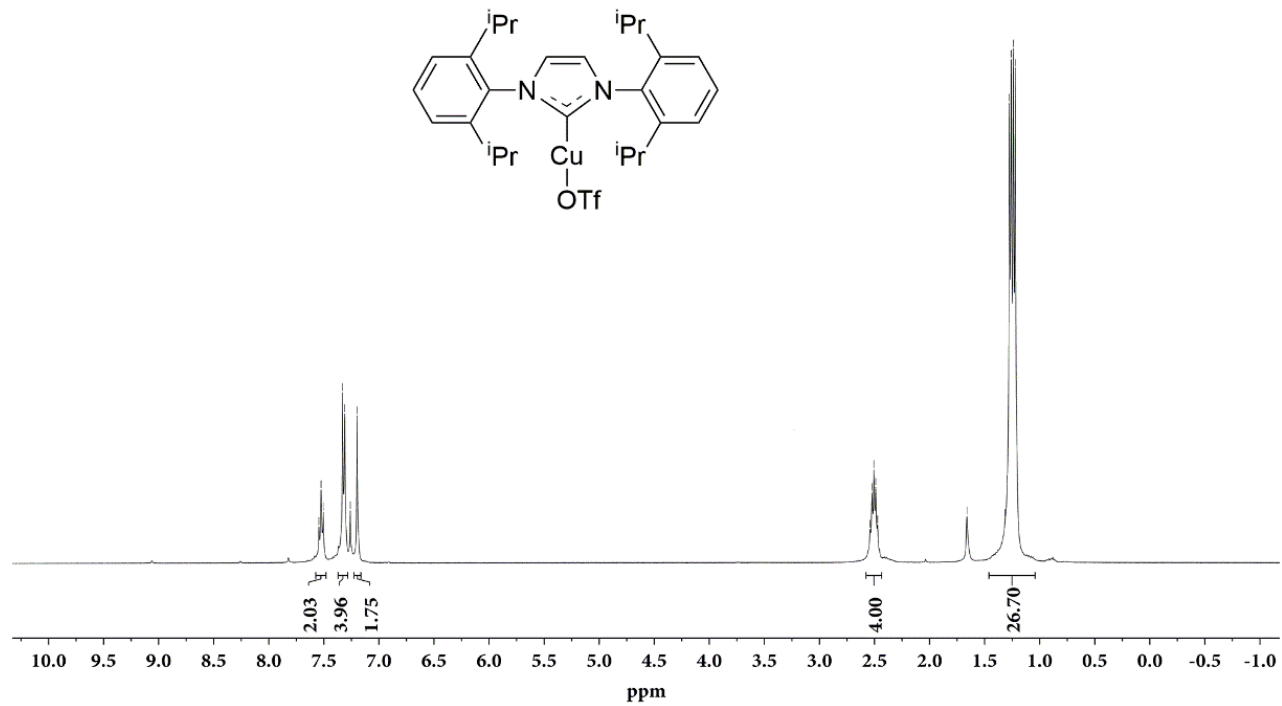

${ }^{1} \mathrm{H}$ NMR (400 MHz) spectra of [1,3-bis(2,6-di-iso-propylphenyl)imidazol-2-ylidene] copper (I) triflate,

$(\mathrm{IPr}) \mathrm{Cu}(\mathrm{OTf})\left(\mathrm{rt}, \mathrm{CDCl}_{3}\right)$. 


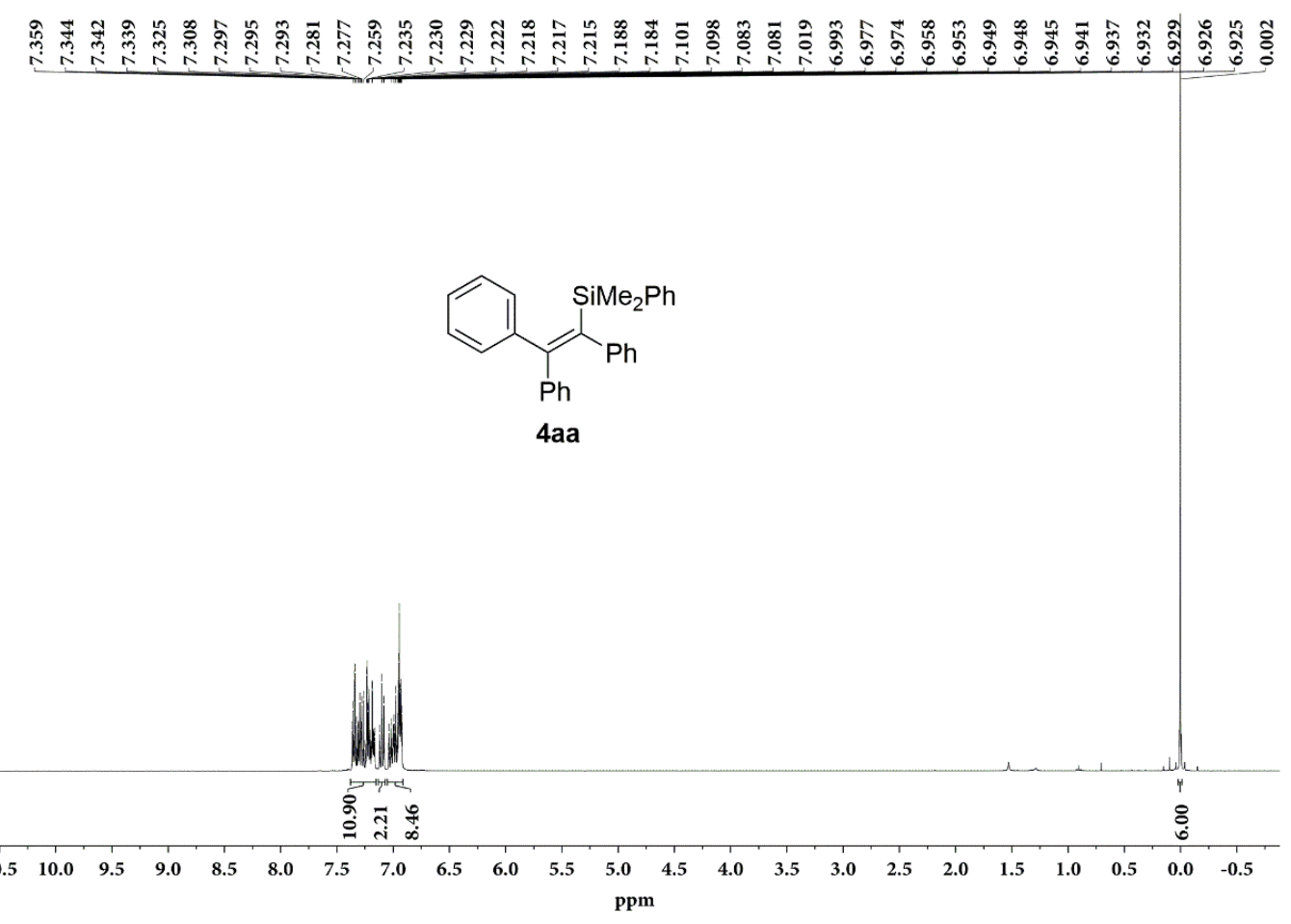

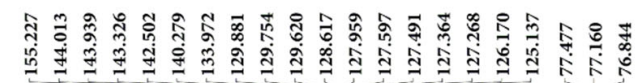

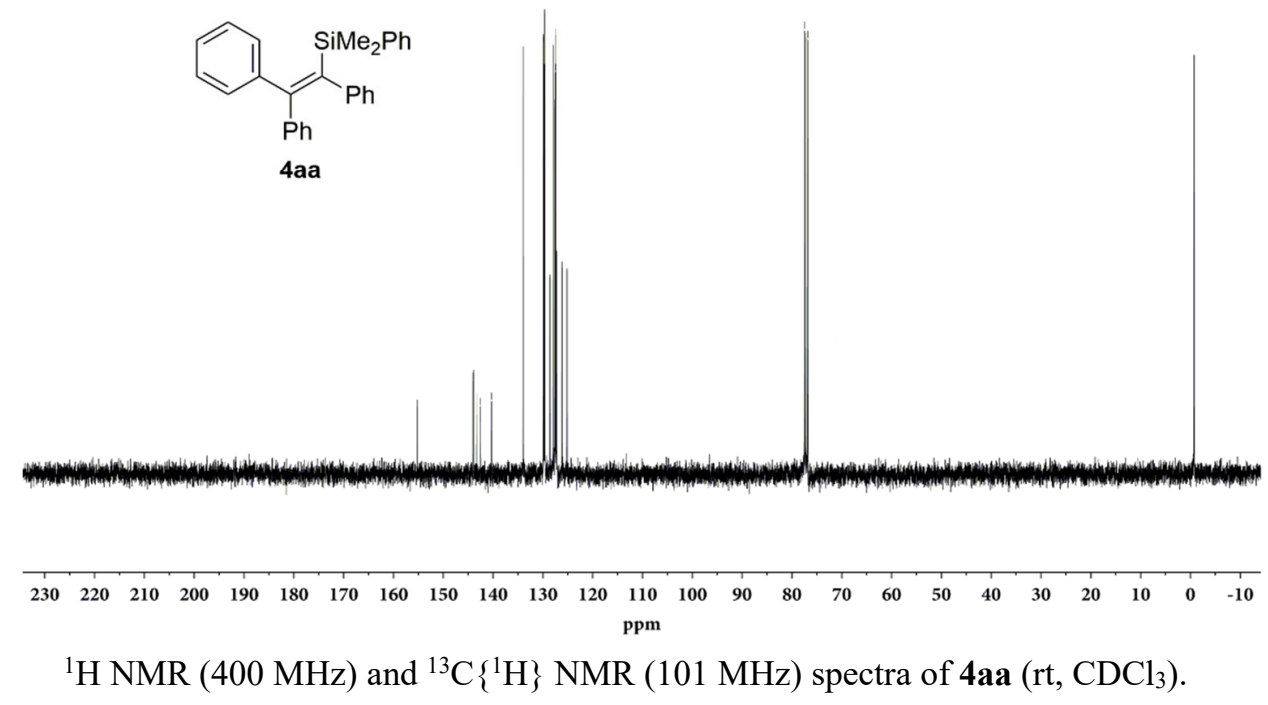



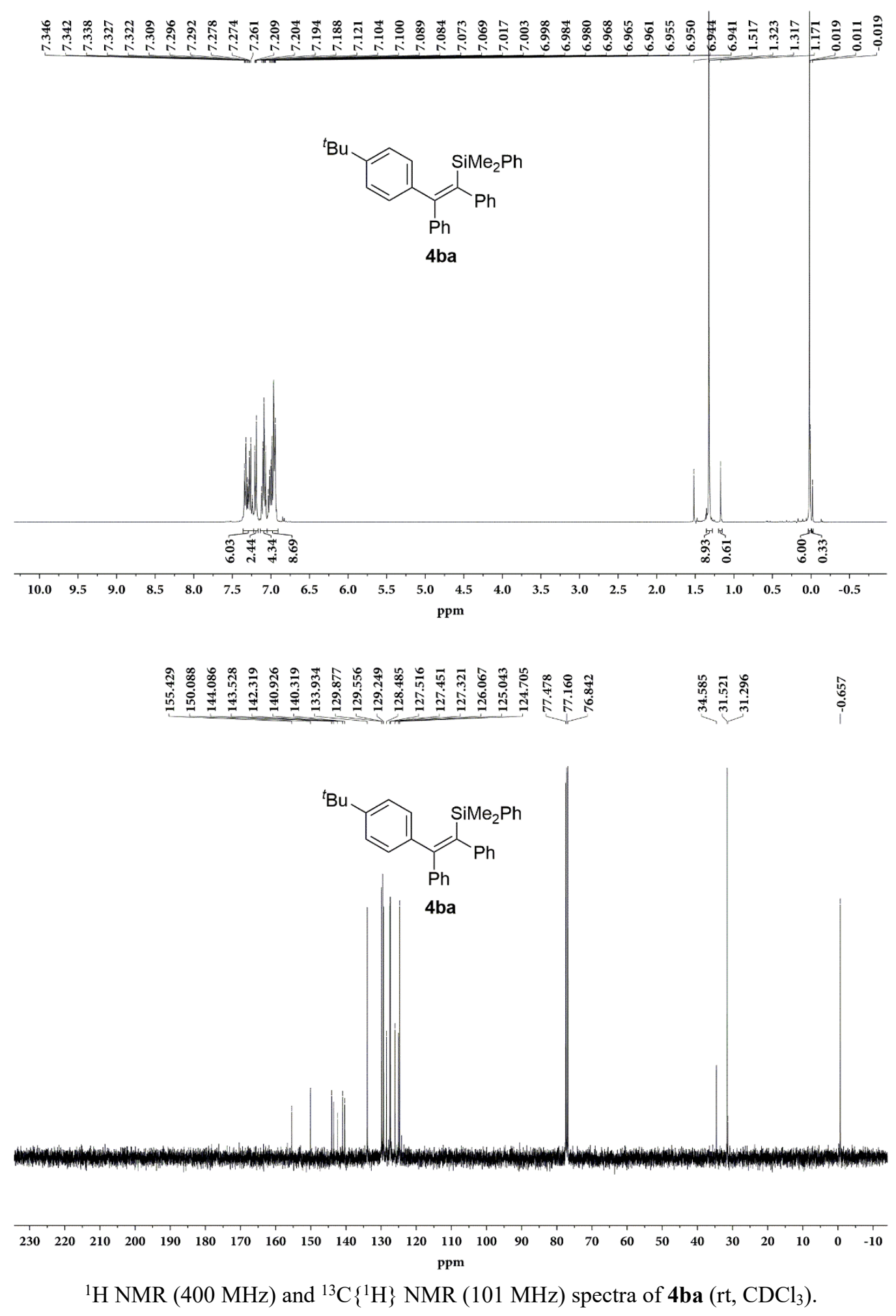


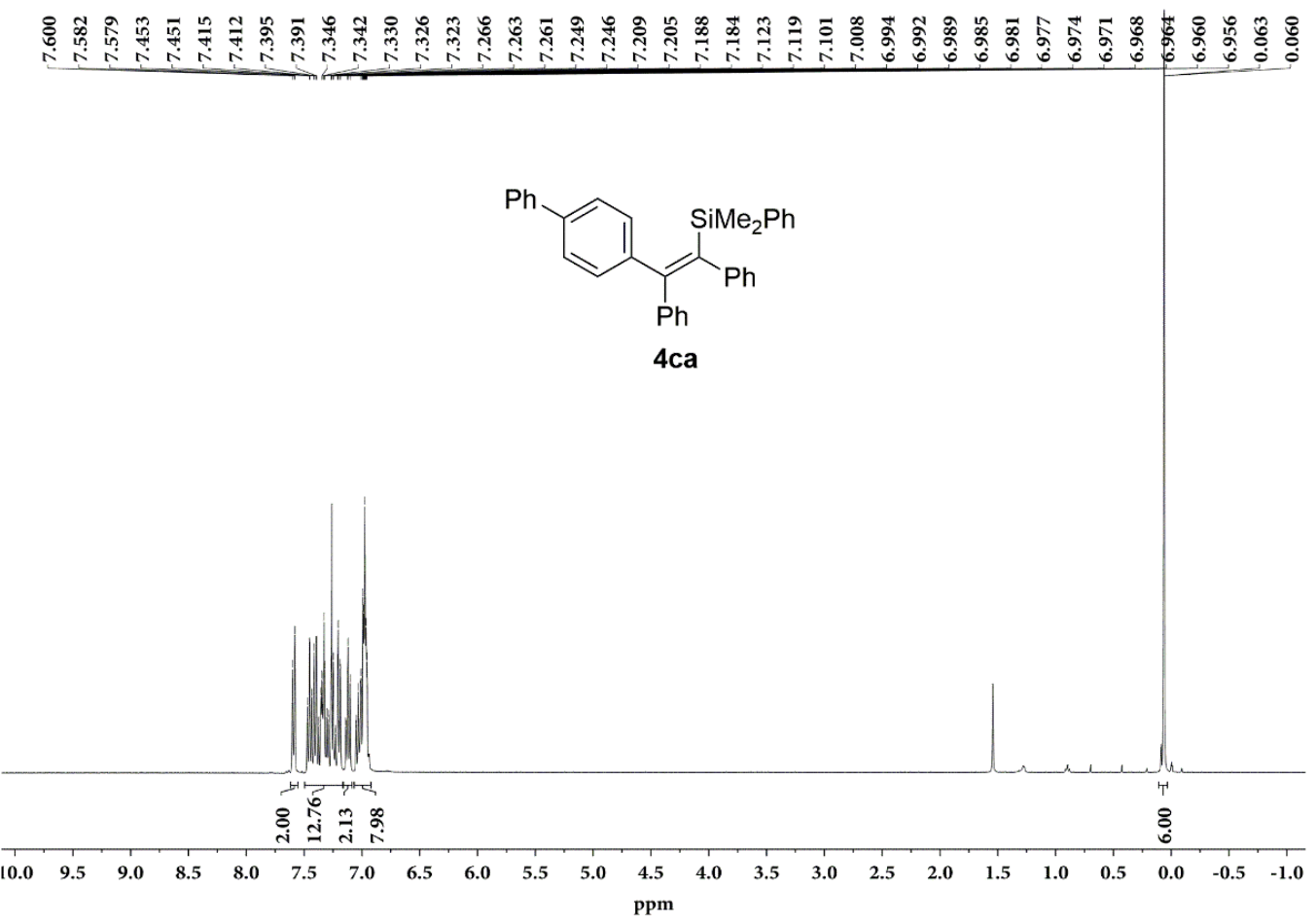

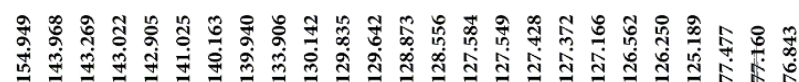

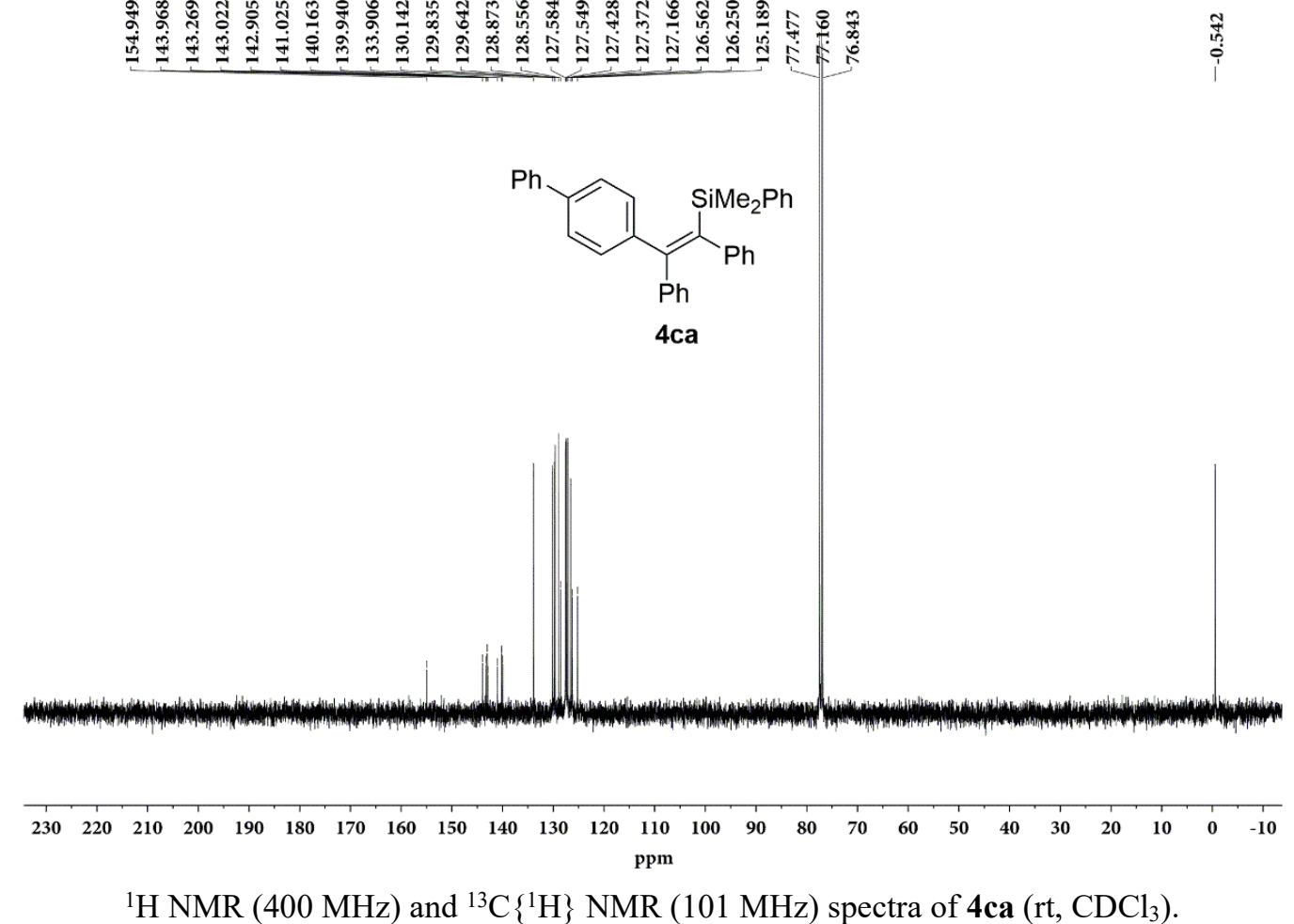

${ }^{1} \mathrm{H}$ NMR $(400 \mathrm{MHz})$ and ${ }^{13} \mathrm{C}\left\{{ }^{1} \mathrm{H}\right\}$ NMR (101 MHz) spectra of $\mathbf{4} \mathbf{c a}\left(\mathrm{rt}, \mathrm{CDCl}_{3}\right)$. 


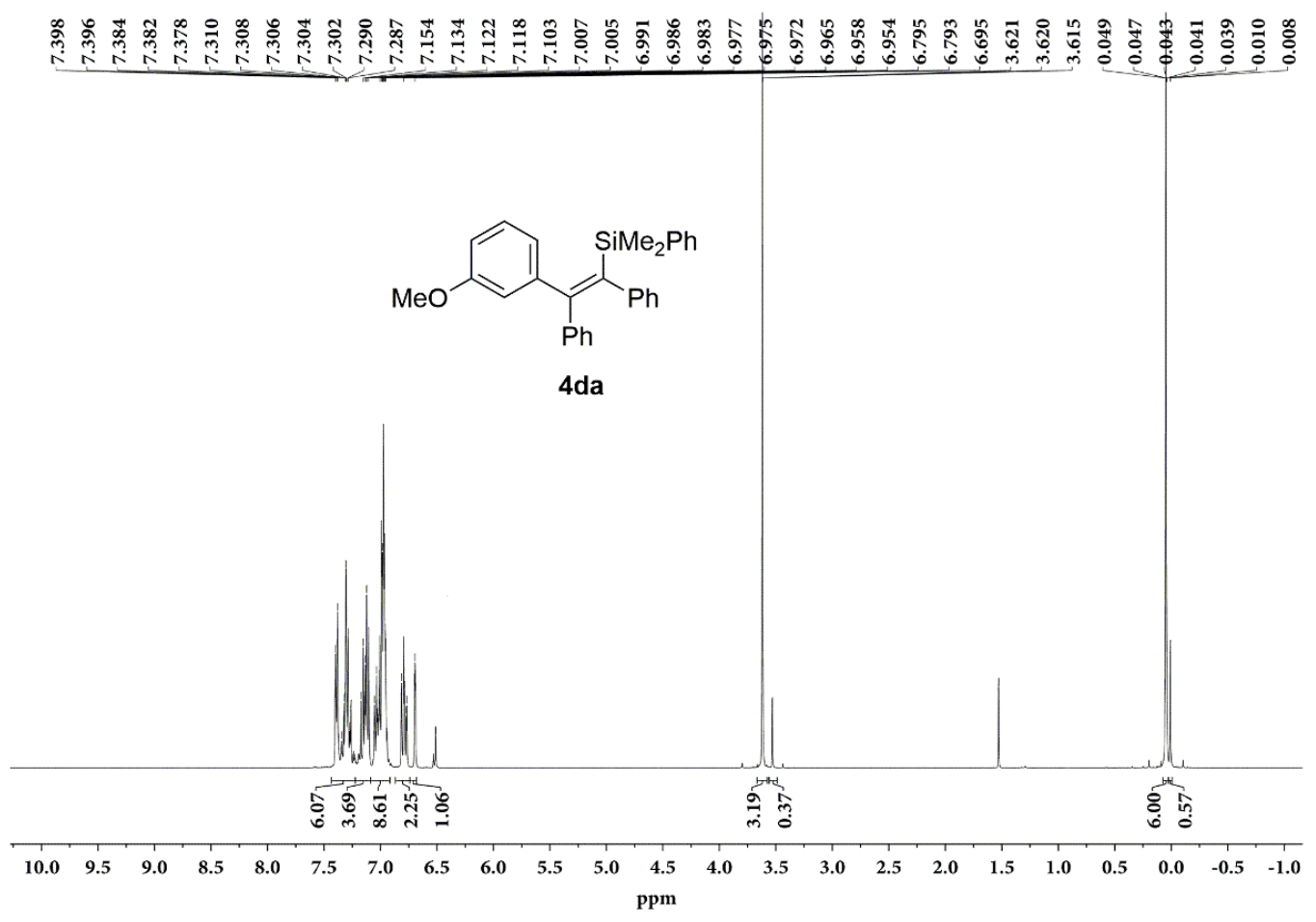

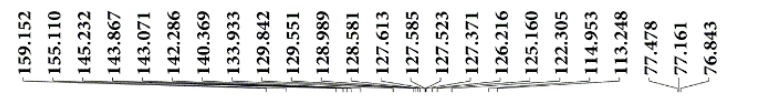

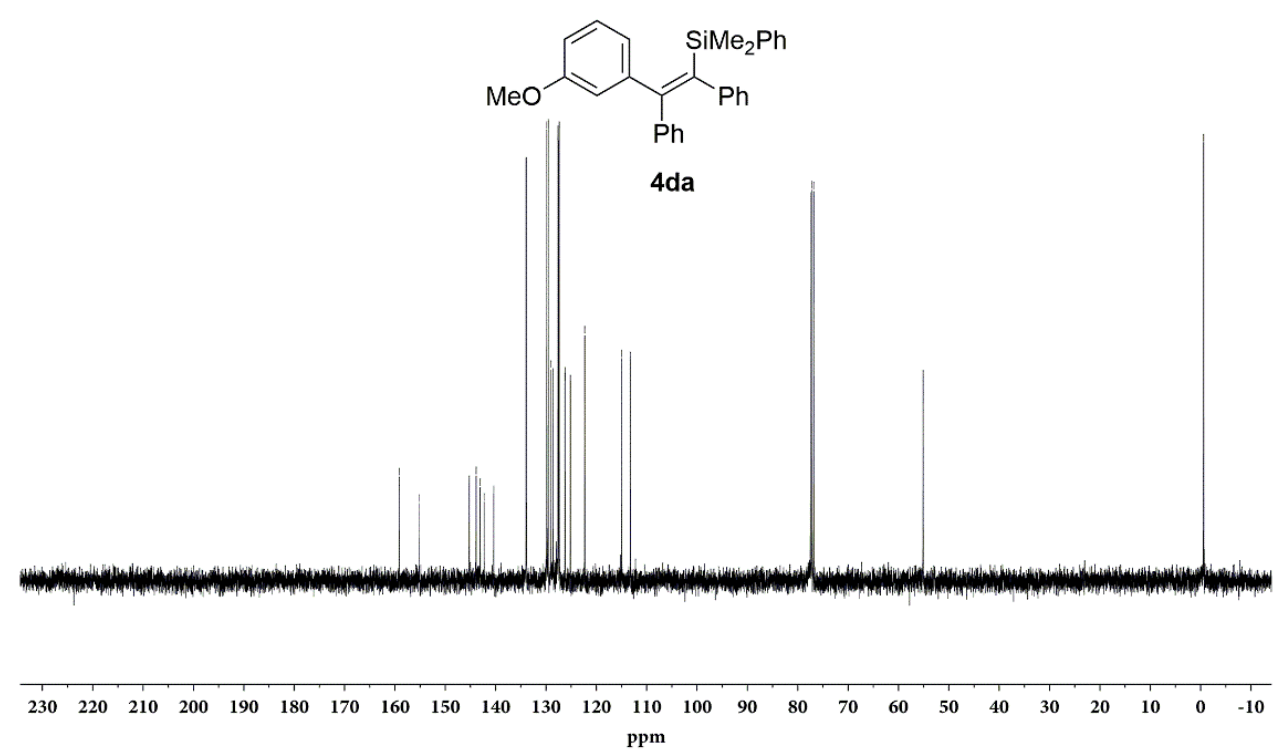

${ }^{1} \mathrm{H}$ NMR (400 MHz) and ${ }^{13} \mathrm{C}\left\{{ }^{1} \mathrm{H}\right\}$ NMR (101 MHz) spectra of 4da (rt, $\left.\mathrm{CDCl}_{3}\right)$. 


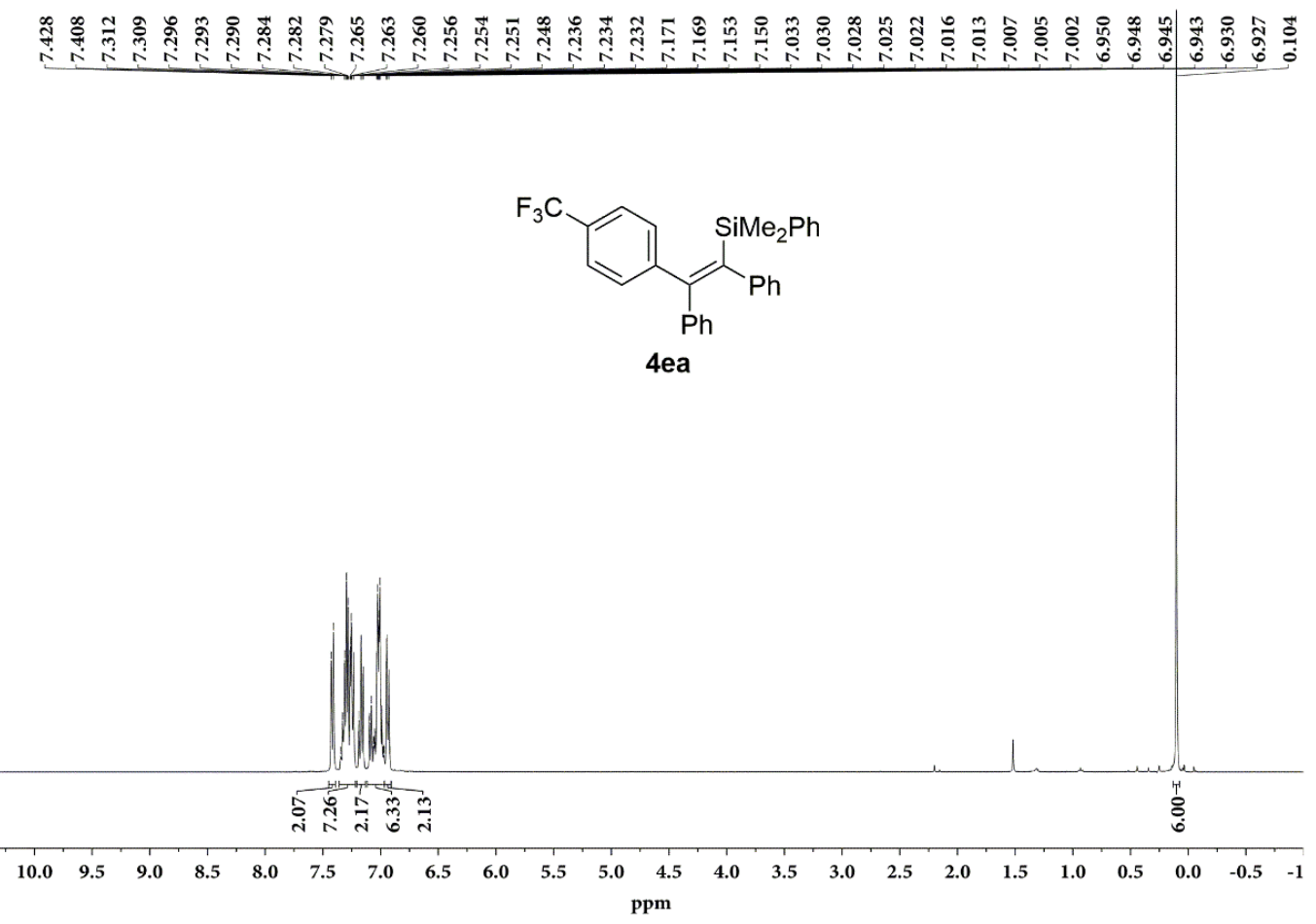

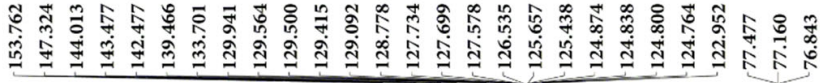

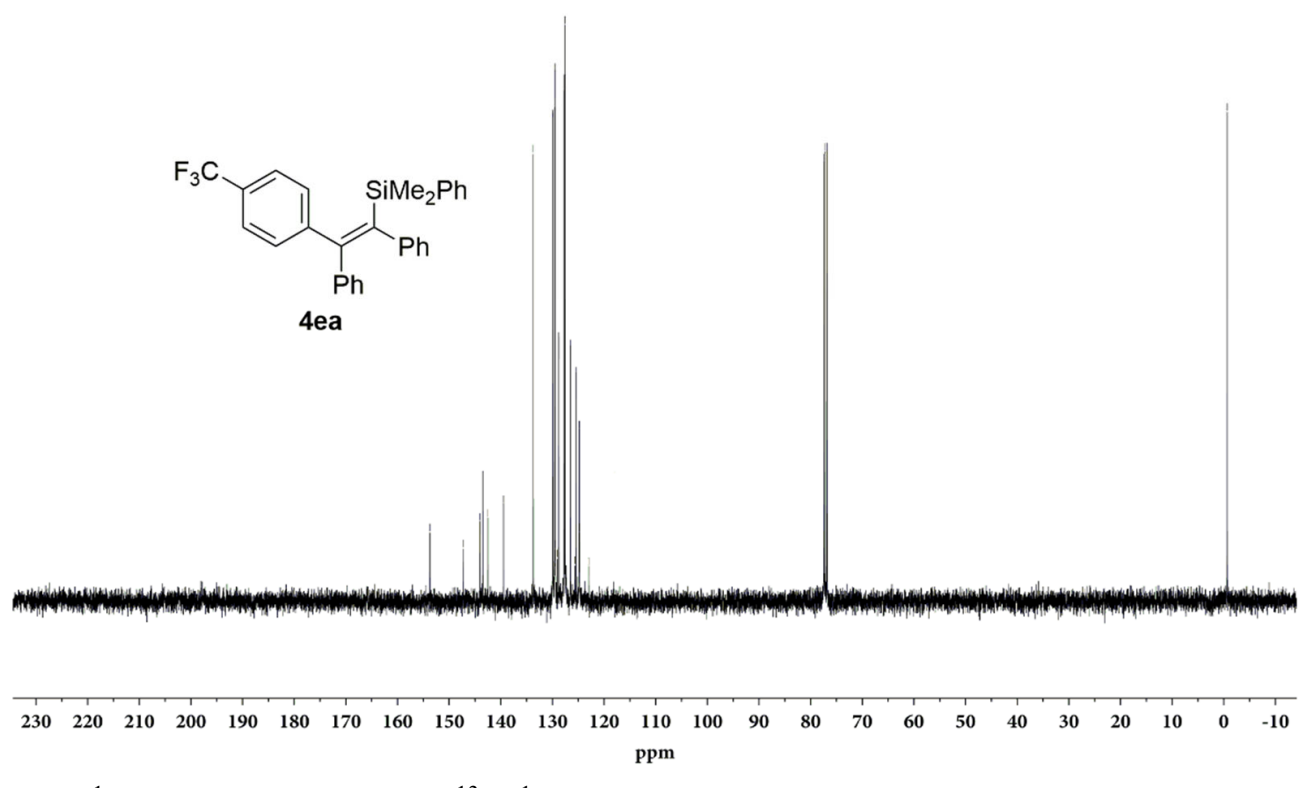

${ }^{1} \mathrm{H}$ NMR $(400 \mathrm{MHz})$ and ${ }^{13} \mathrm{C}\left\{{ }^{1} \mathrm{H}\right\}$ NMR $(101 \mathrm{MHz})$ spectra of $4 \mathbf{e a}\left(\mathrm{rt}, \mathrm{CDCl}_{3}\right)$. 


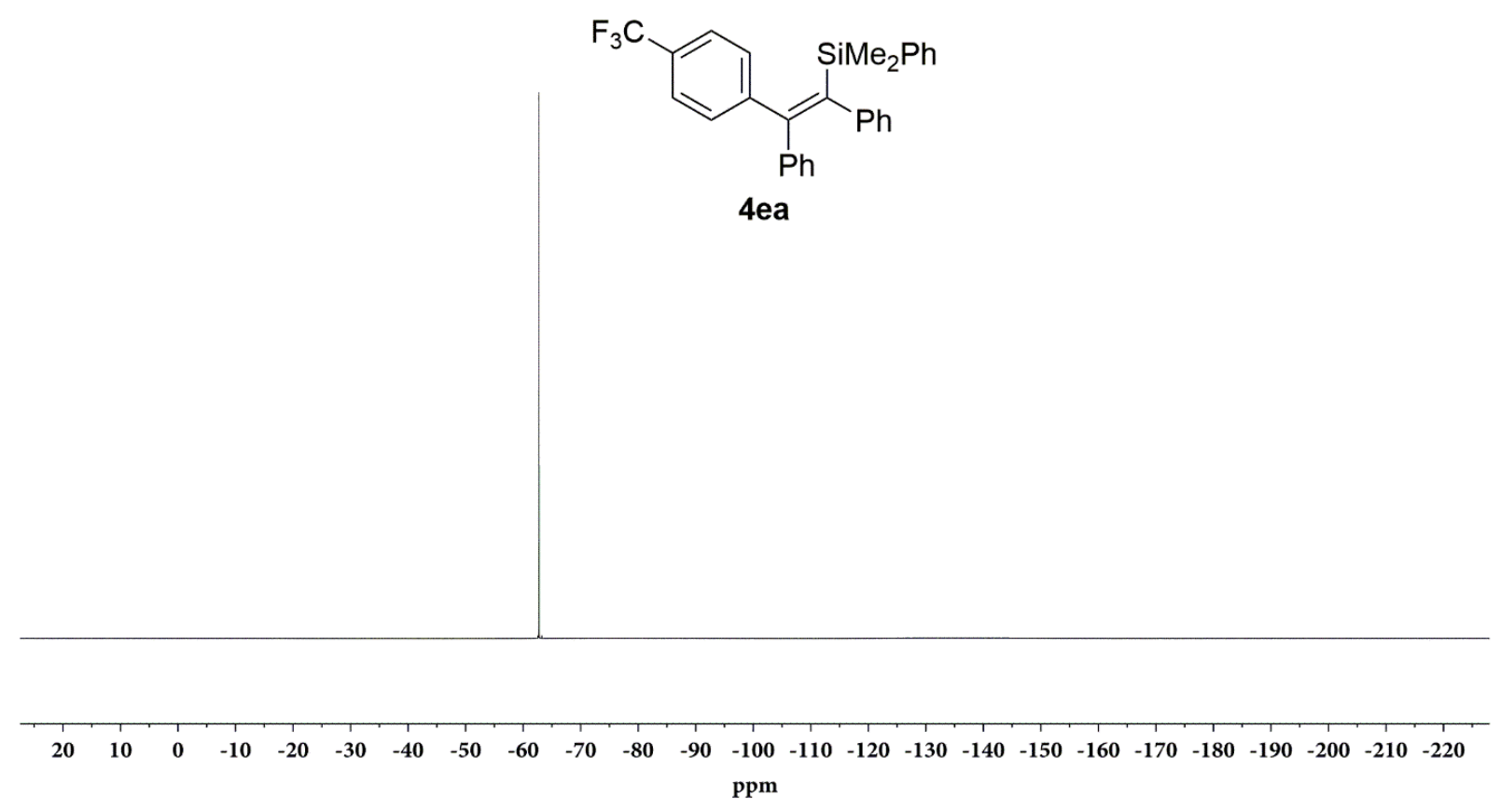

${ }^{19} \mathrm{~F}\left\{{ }^{1} \mathrm{H}\right\}$ NMR $(376 \mathrm{MHz})$ spectrum of $4 \mathbf{e a}\left(\mathrm{rt}, \mathrm{CDCl}_{3}\right)$. 

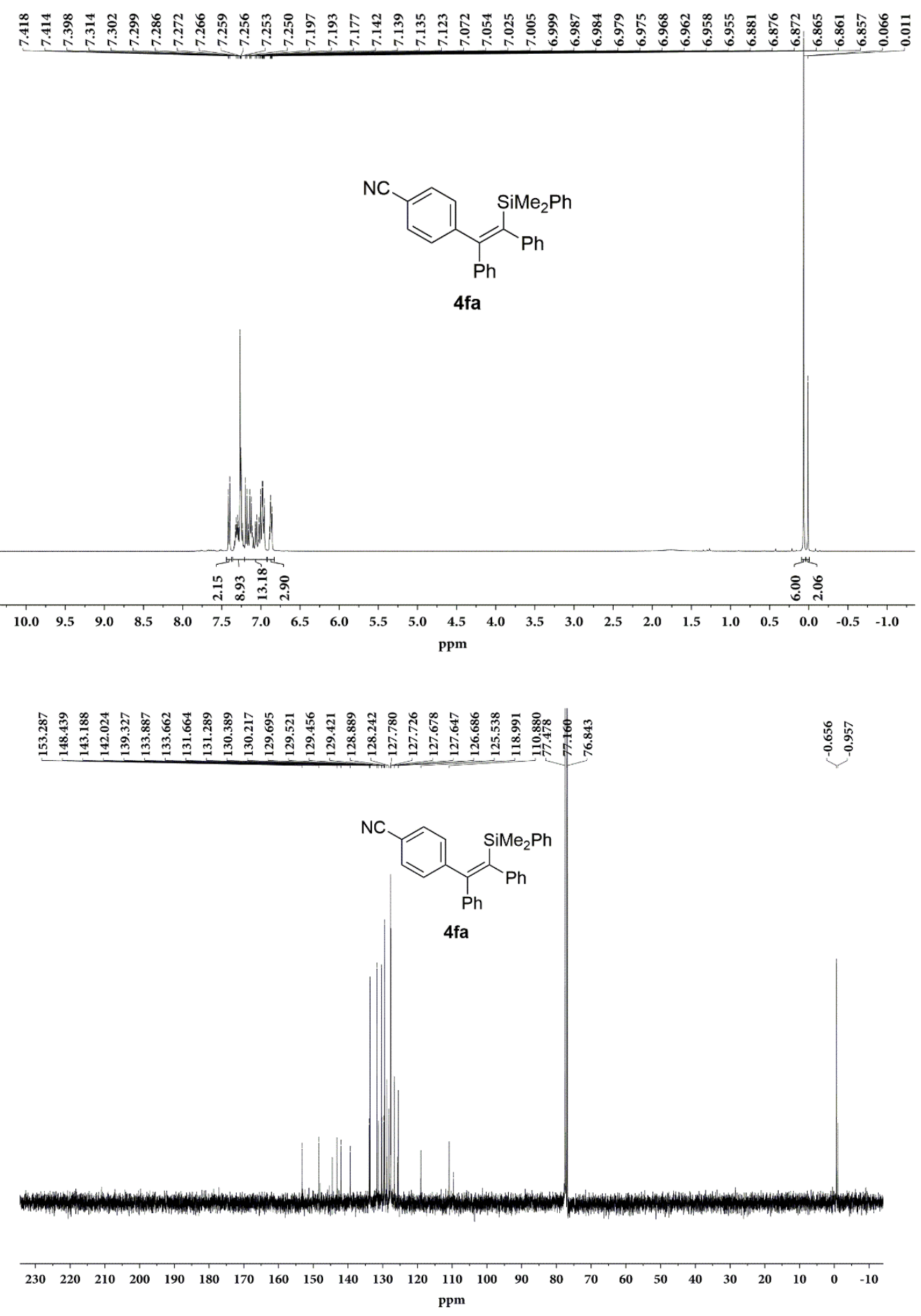

${ }^{1} \mathrm{H}$ NMR (400 MHz) and ${ }^{13} \mathrm{C}\left\{{ }^{1} \mathrm{H}\right\}$ NMR $(101 \mathrm{MHz})$ spectra of $\mathbf{4 f a}\left(\mathrm{rt}, \mathrm{CDCl}_{3}\right)$. 


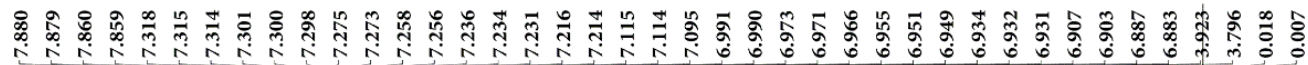

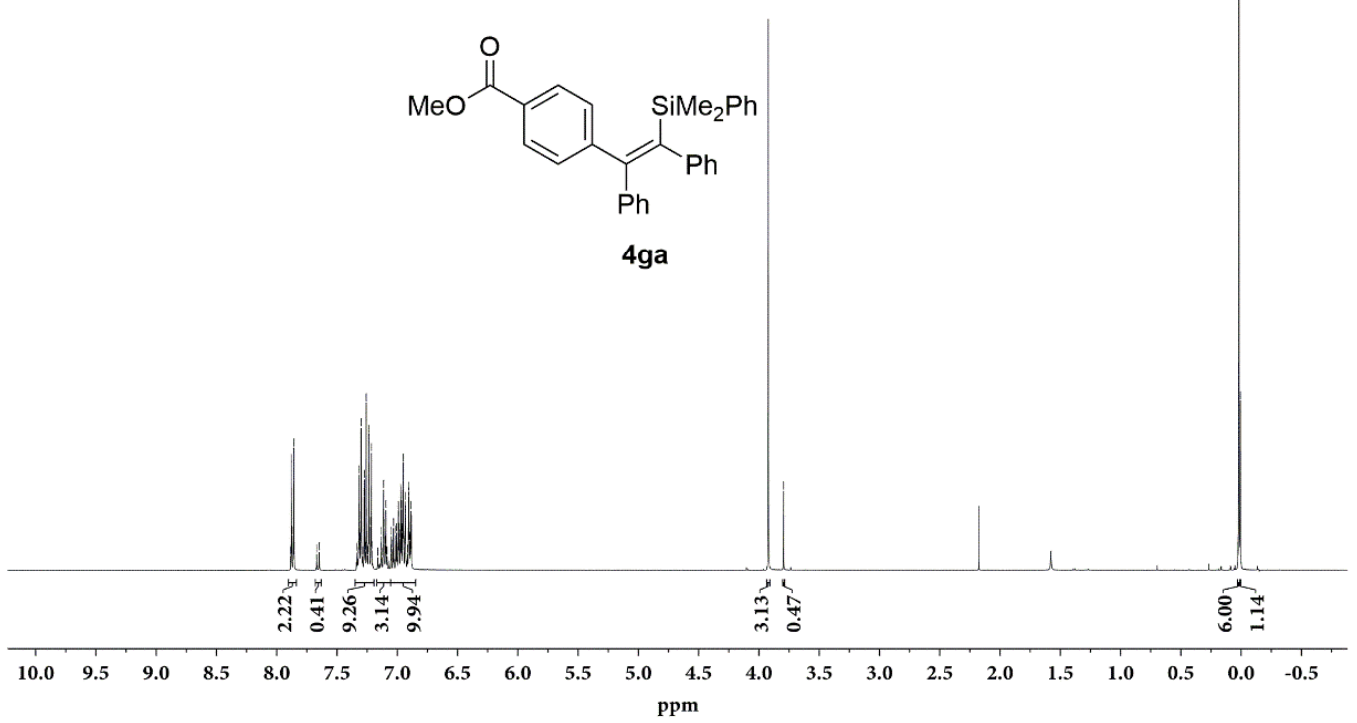

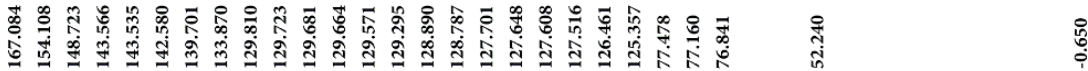

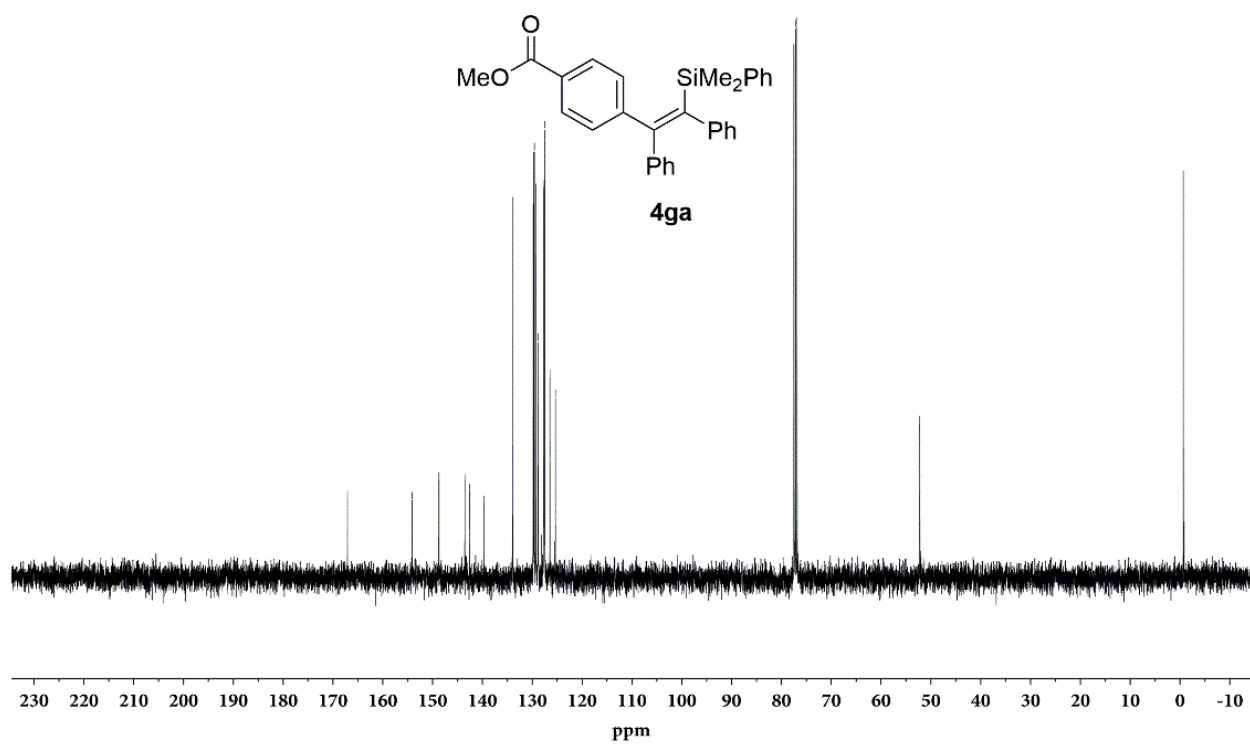

${ }^{1} \mathrm{H}$ NMR (400 MHz) and ${ }^{13} \mathrm{C}\left\{{ }^{1} \mathrm{H}\right\}$ NMR (101 MHz) spectra of 4ga (rt, $\left.\mathrm{CDCl}_{3}\right)$. 


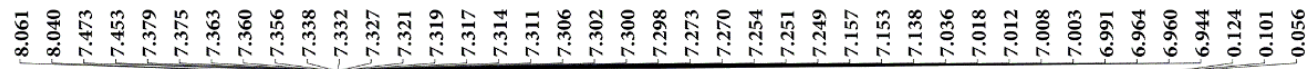

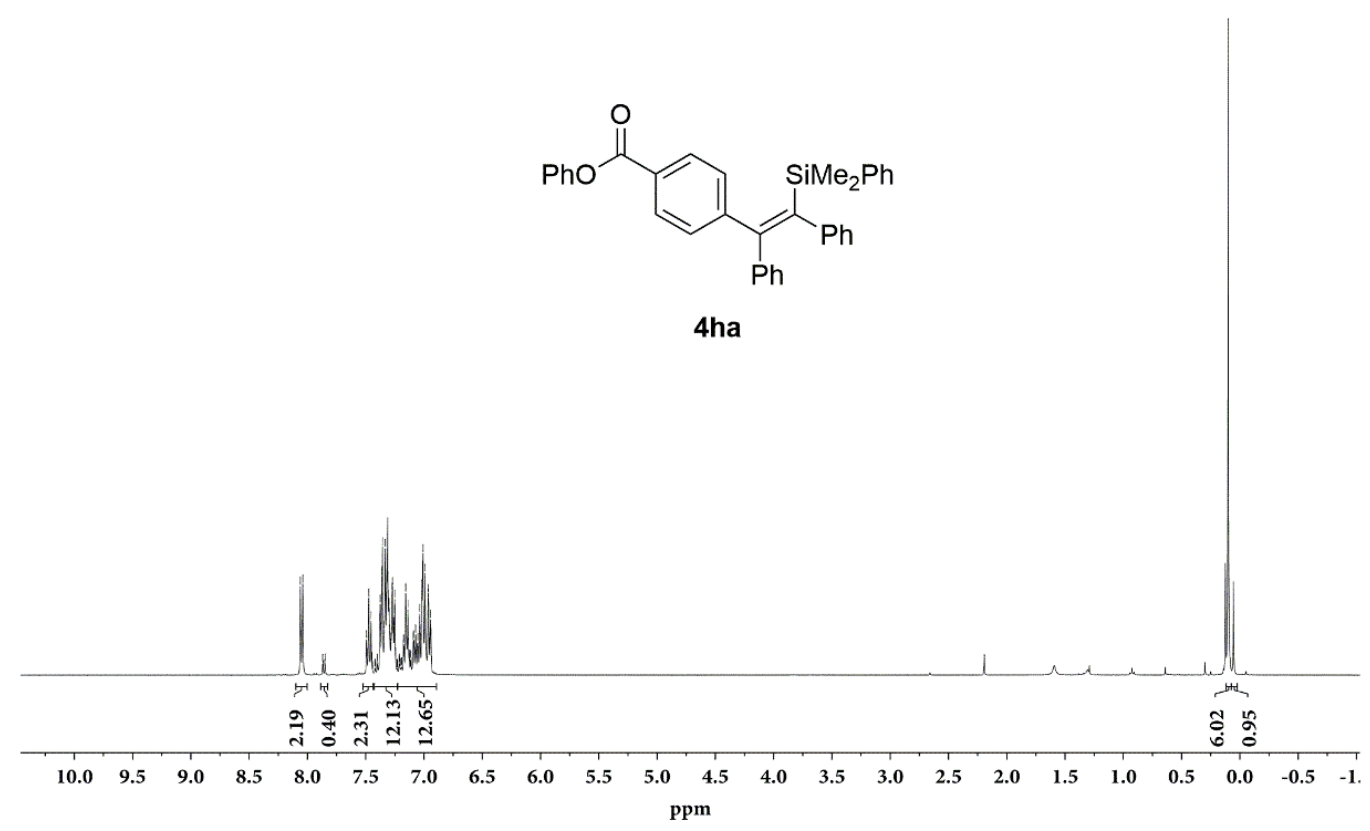

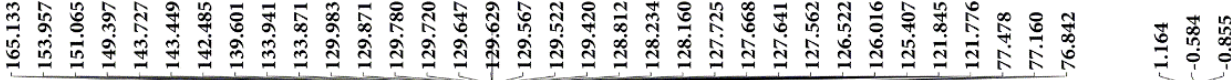<smiles>O=C(Oc1ccccc1)c1ccc(C(=C(c2ccccc2)c2ccccc2)c2ccccc2)cc1</smiles>

4ha

$\begin{array}{lllllllllllllllllllllllll}230 & 220 & 210 & 200 & 190 & 180 & 170 & 160 & 150 & 140 & 130 & 120 & 110 & 100 & 90 & 80 & 70 & 60 & 50 & 40 & 30 & 20 & 10 & 0 & -10\end{array}$

${ }^{1} \mathrm{H}$ NMR (400 MHz) and ${ }^{13} \mathrm{C}\left\{{ }^{1} \mathrm{H}\right\}$ NMR (101 MHz) spectra of 4ha (rt, $\left.\mathrm{CDCl}_{3}\right)$. 


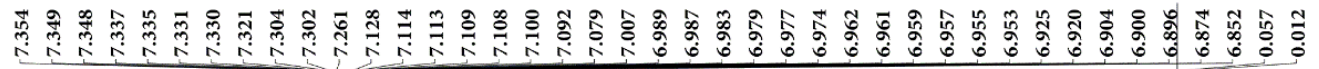

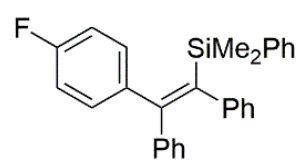

4 ia

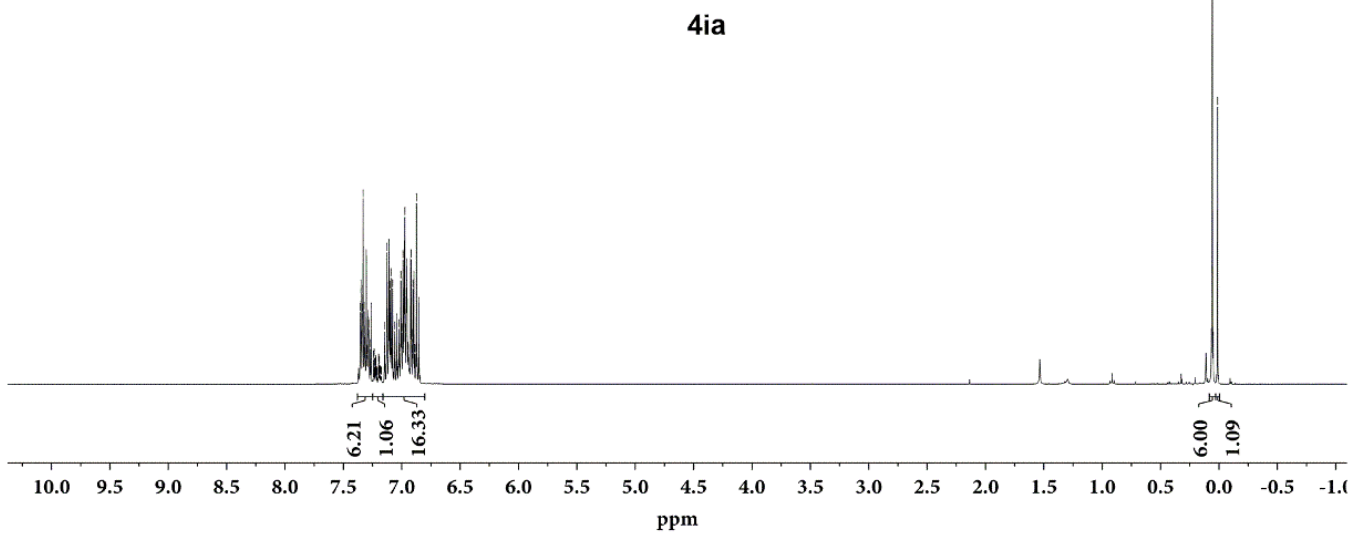

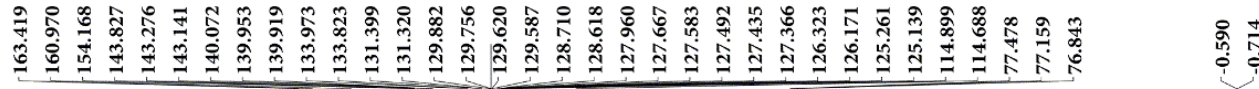

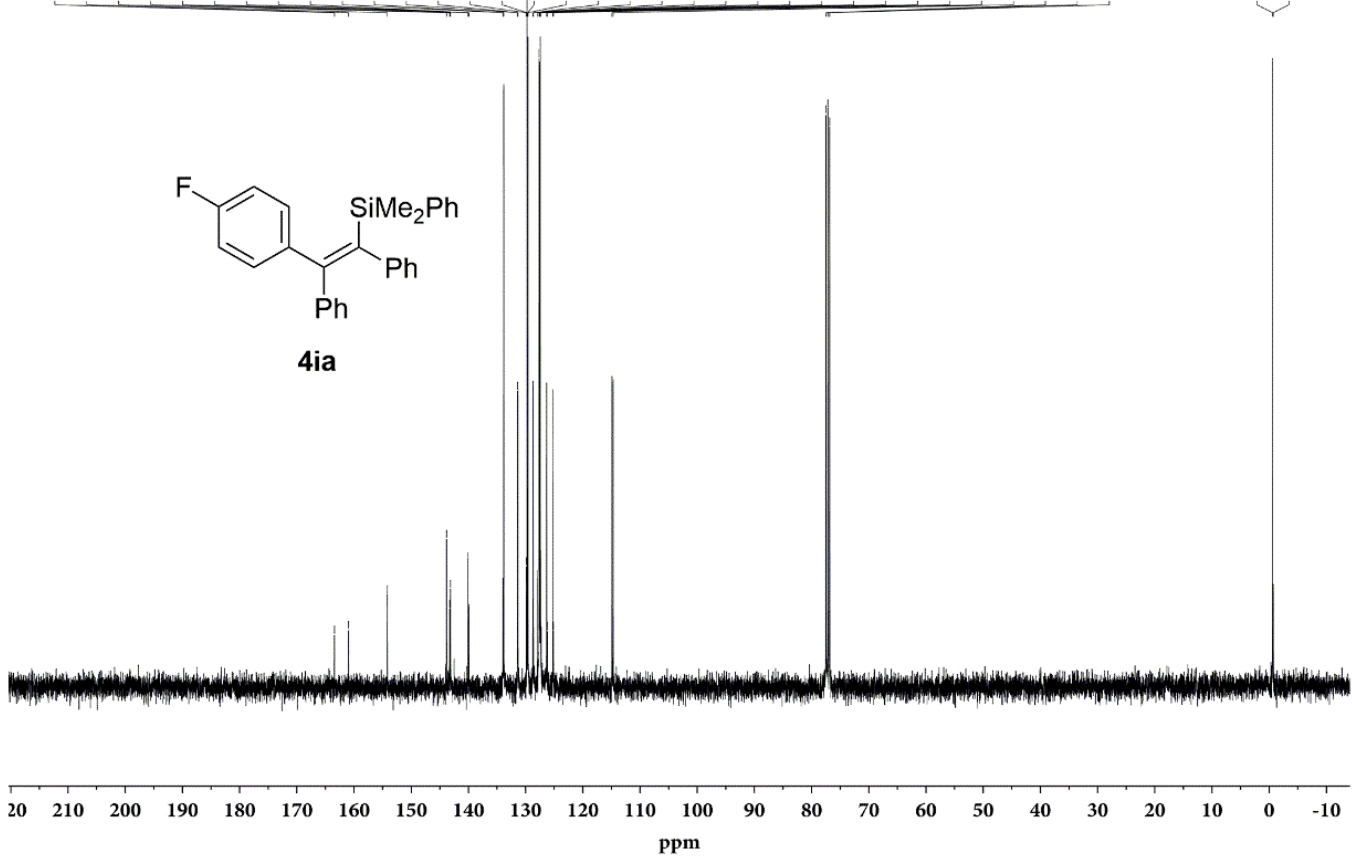

${ }^{1} \mathrm{H}$ NMR $(400 \mathrm{MHz})$ and ${ }^{13} \mathrm{C}\left\{{ }^{1} \mathrm{H}\right\}$ NMR (101 MHz) spectra of $\mathbf{4 i a}\left(\mathrm{rt}, \mathrm{CDCl}_{3}\right)$. 


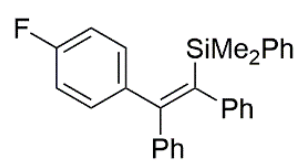

4ia

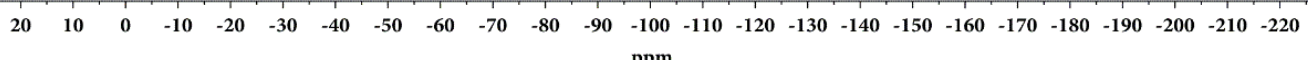

${ }^{19} \mathrm{~F}\left\{{ }^{1} \mathrm{H}\right\}$ NMR $(376 \mathrm{MHz})$ spectrum of $4 \mathbf{i a}\left(\mathrm{rt}, \mathrm{CDCl}_{3}\right)$. 

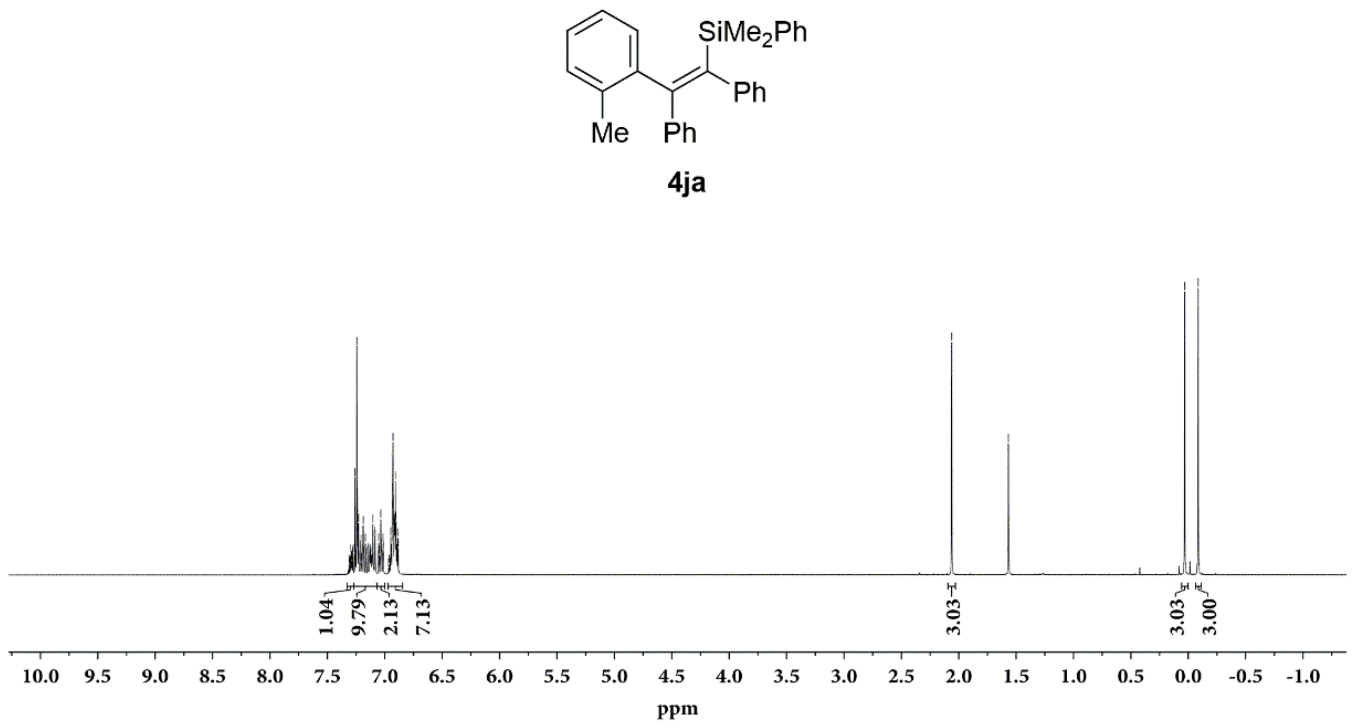

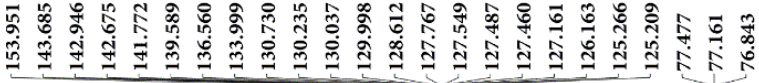

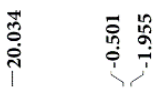

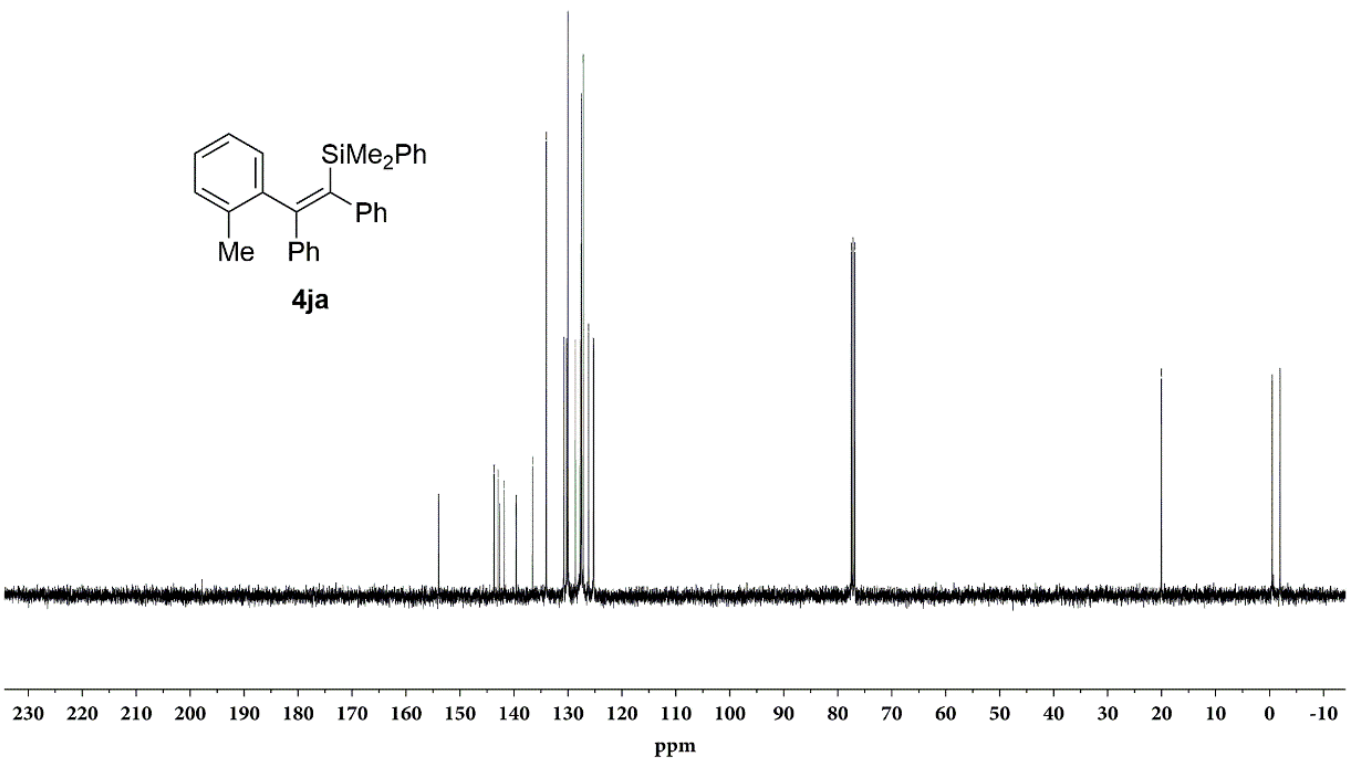

${ }^{1} \mathrm{H}$ NMR (400 MHz) and ${ }^{13} \mathrm{C}\left\{{ }^{1} \mathrm{H}\right\}$ NMR (101 MHz) spectra of $\mathbf{4 j a}\left(\mathrm{rt}, \mathrm{CDCl}_{3}\right)$. 


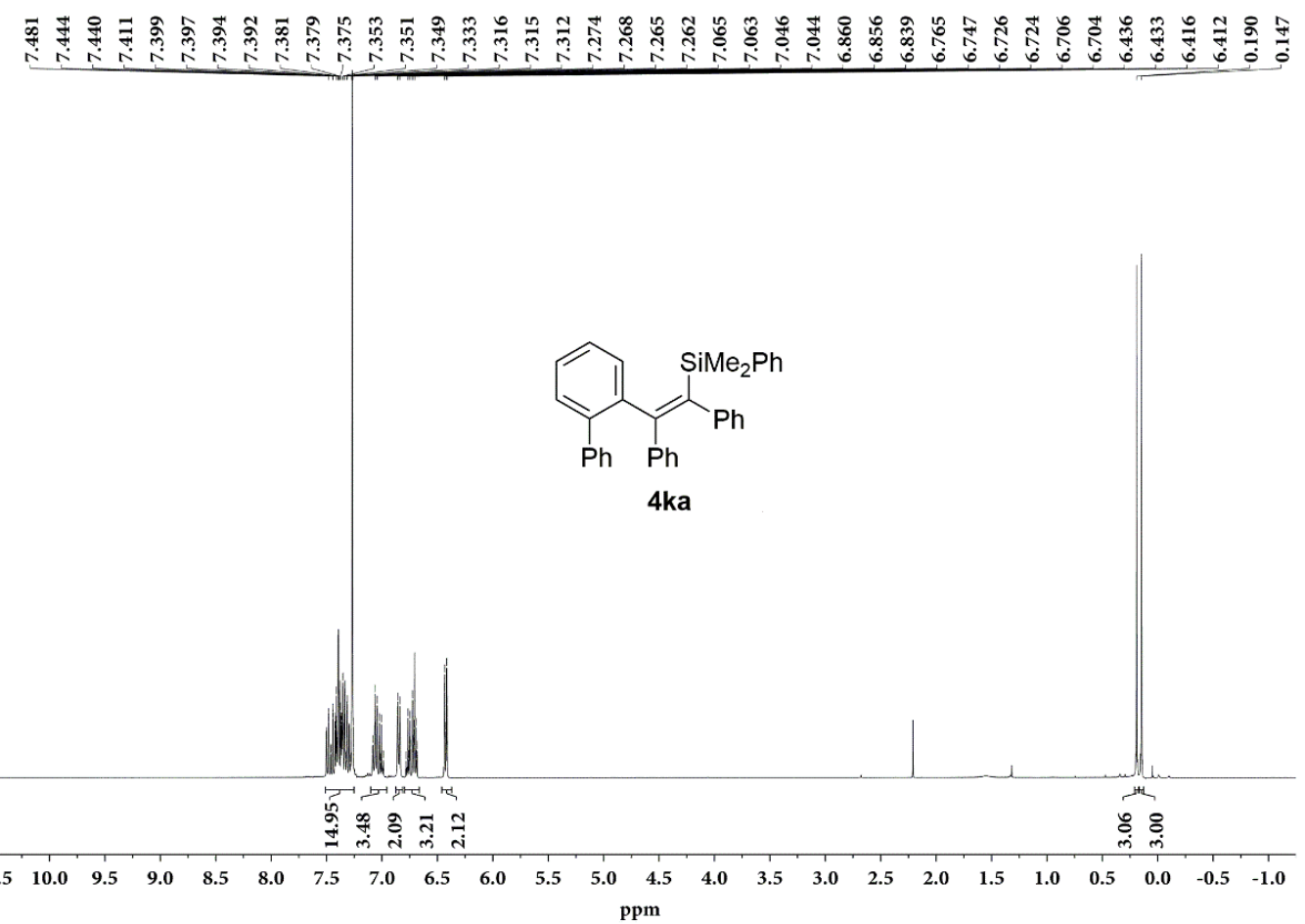

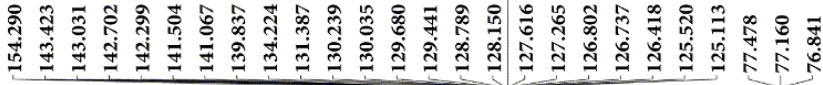

$\begin{array}{lllllllllllllllllllllllll}230 & 220 & 210 & 200 & 190 & 180 & 170 & 160 & 150 & 140 & 130 & 120 & 110 & 100 & 90 & 80 & 70 & 60 & 50 & 40 & 30 & 20 & 10 & 0 & -10\end{array}$ $\mathrm{ppm}$

${ }^{1} \mathrm{H}$ NMR (400 MHz) and ${ }^{13} \mathrm{C}\left\{{ }^{1} \mathrm{H}\right\}$ NMR $(101 \mathrm{MHz})$ spectra of $4 \mathbf{k a}\left(\mathrm{rt}, \mathrm{CDCl}_{3}\right)$. 


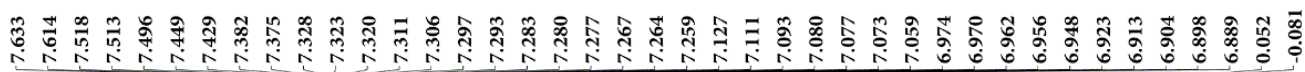

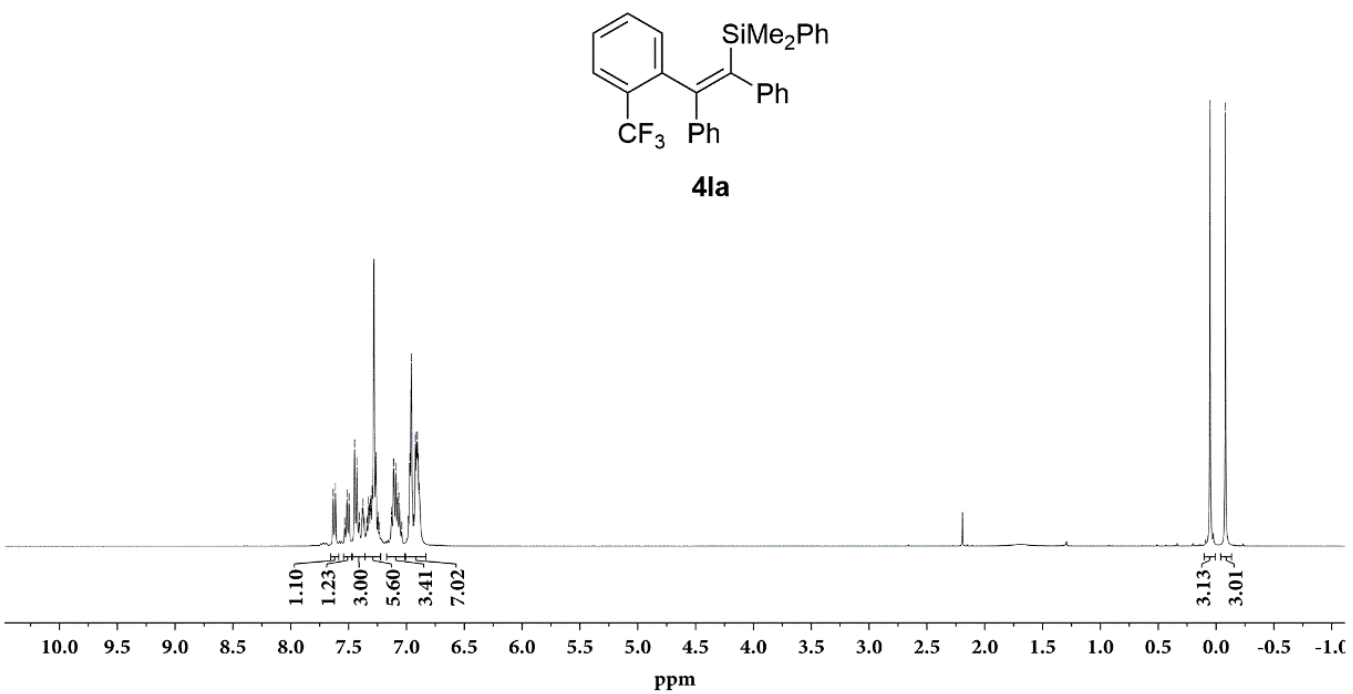

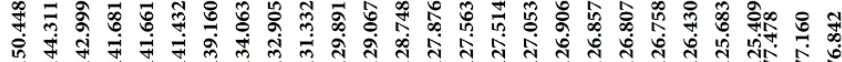

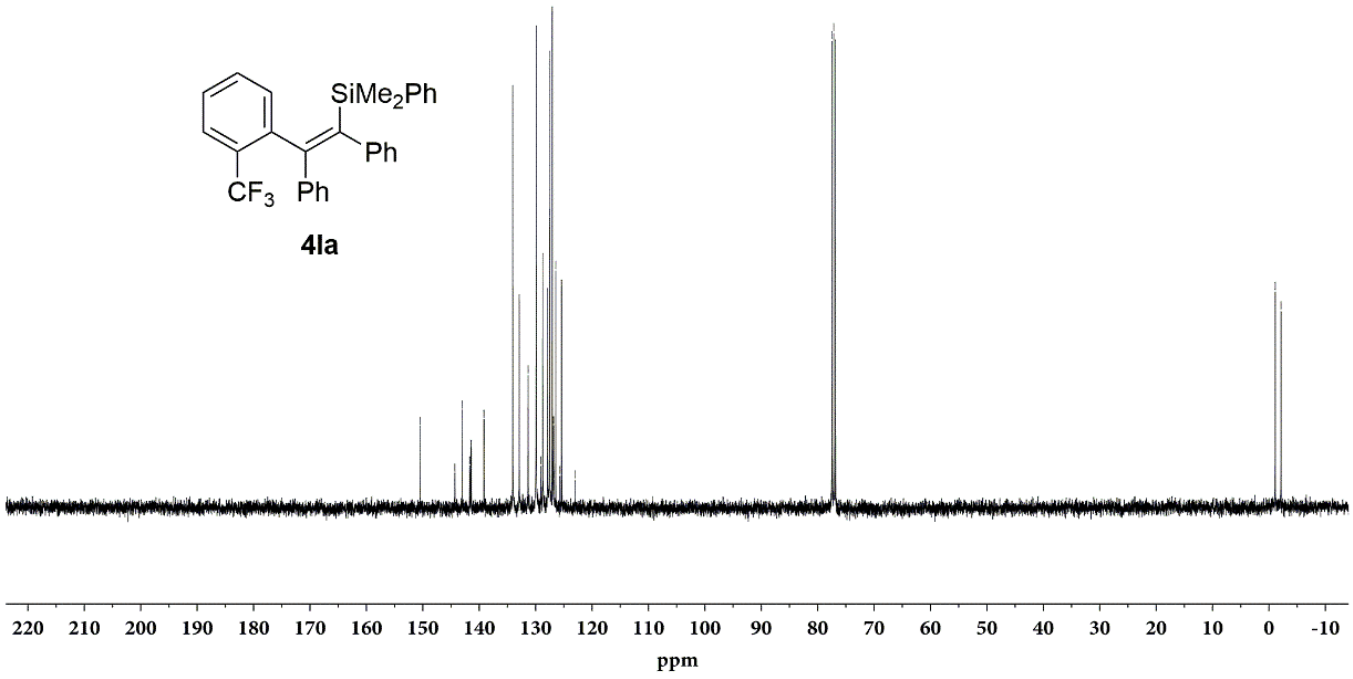

${ }^{1} \mathrm{H}$ NMR (400 MHz) and ${ }^{13} \mathrm{C}\left\{{ }^{1} \mathrm{H}\right\}$ NMR (101 MHz) spectra of 4 la (rt, $\left.\mathrm{CDCl}_{3}\right)$. 


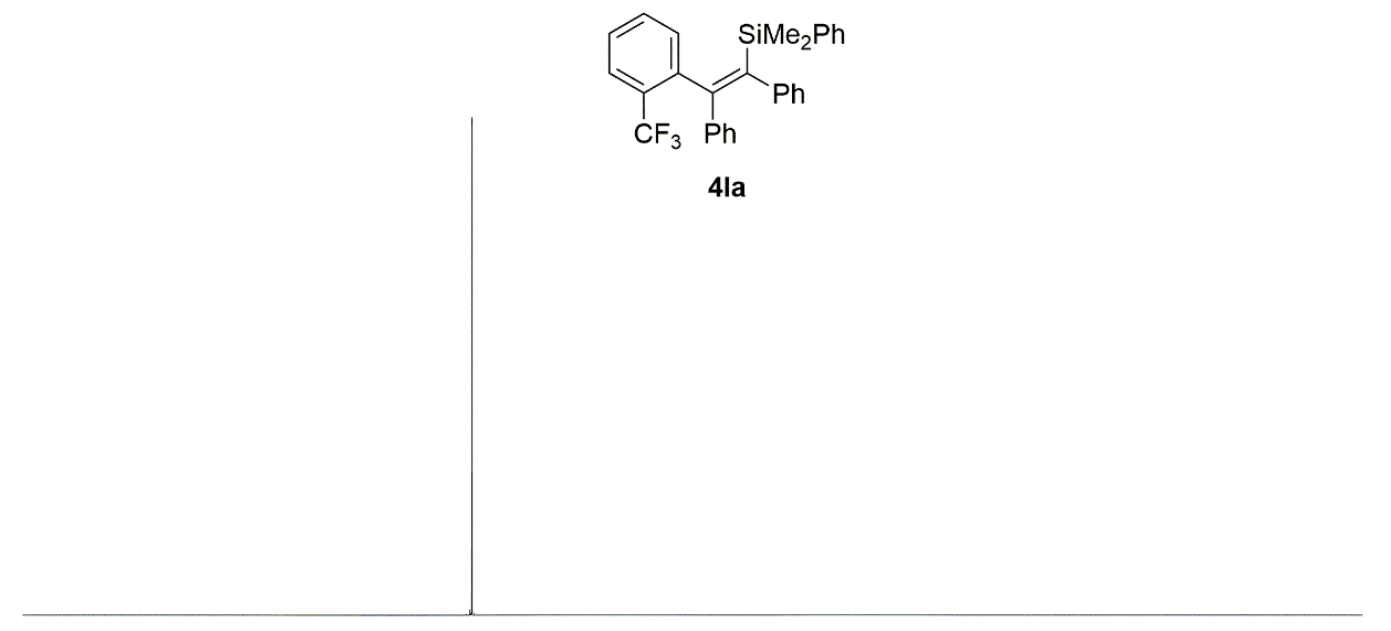

$\begin{array}{lllllllllllllllllllllllllllll}20 & 10 & 0 & -10 & -20 & -30 & -40 & -50 & -60 & -70 & -80 & -90 & -100 & -110 & -120 & -130 & -140 & -150 & -160 & -170 & -180 & -190 & -200 & -210 & -220\end{array}$ ppm

${ }^{19} \mathrm{~F}\left\{{ }^{1} \mathrm{H}\right\}$ NMR $(376 \mathrm{MHz})$ spectrum of $4 \mathbf{l a}\left(\mathrm{rt}, \mathrm{CDCl}_{3}\right)$. 


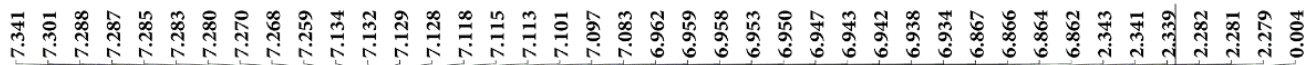

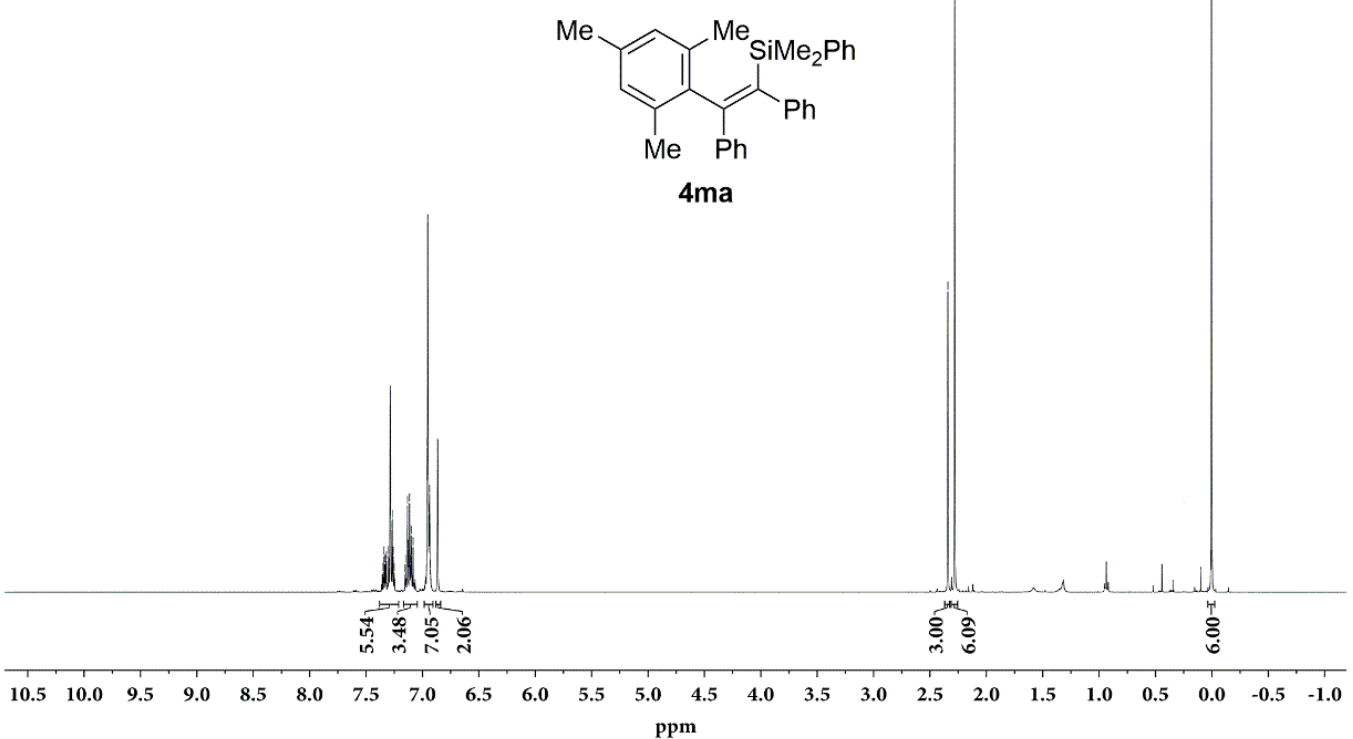

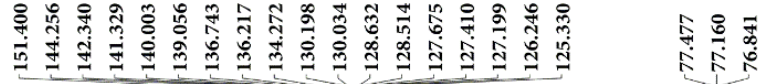

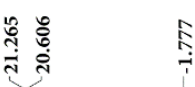
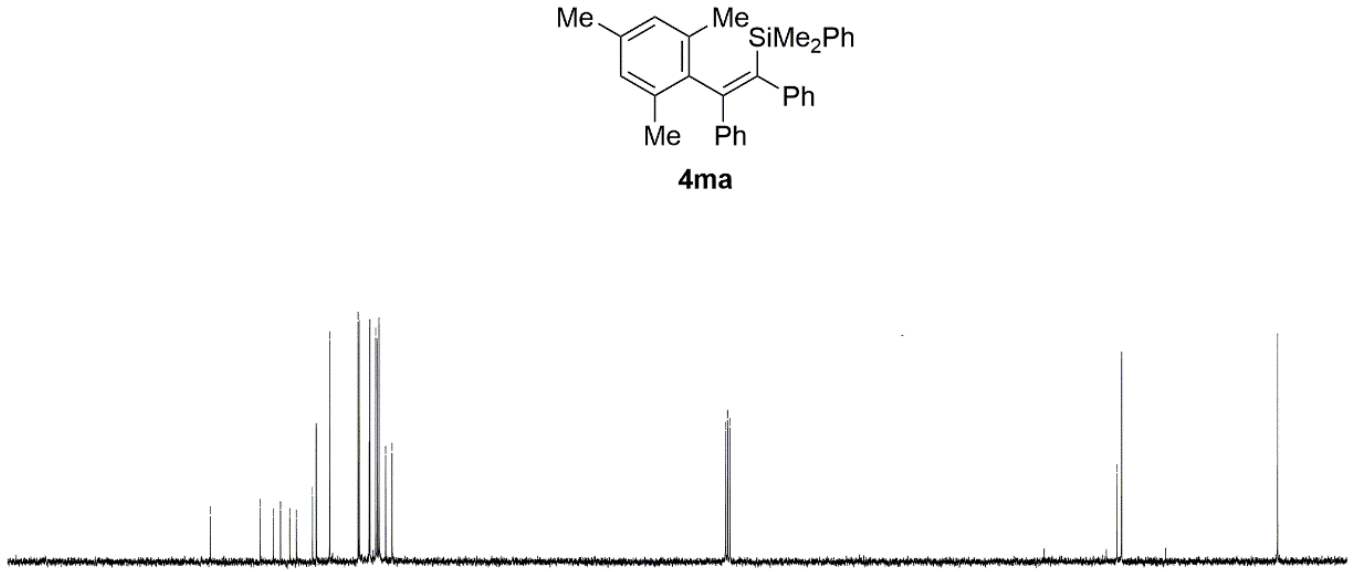

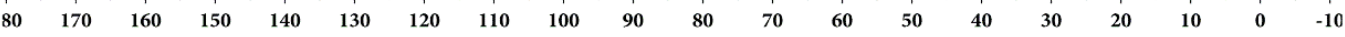

${ }^{1} \mathrm{H}$ NMR (400 MHz) and ${ }^{13} \mathrm{C}\left\{{ }^{1} \mathrm{H}\right\}$ NMR (101 MHz) spectra of 4ma (rt, $\left.\mathrm{CDCl}_{3}\right)$. 


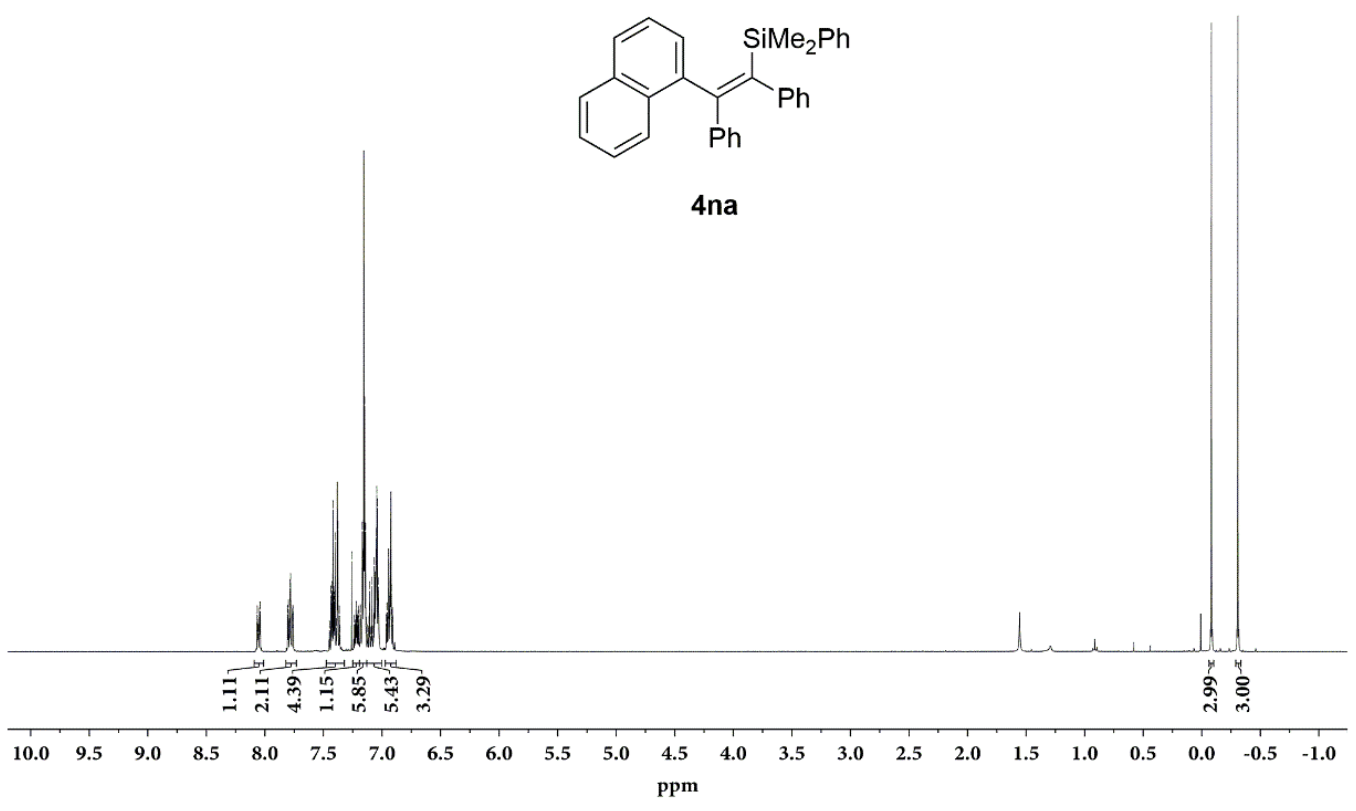

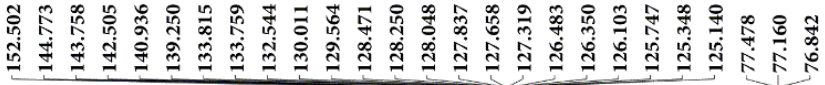

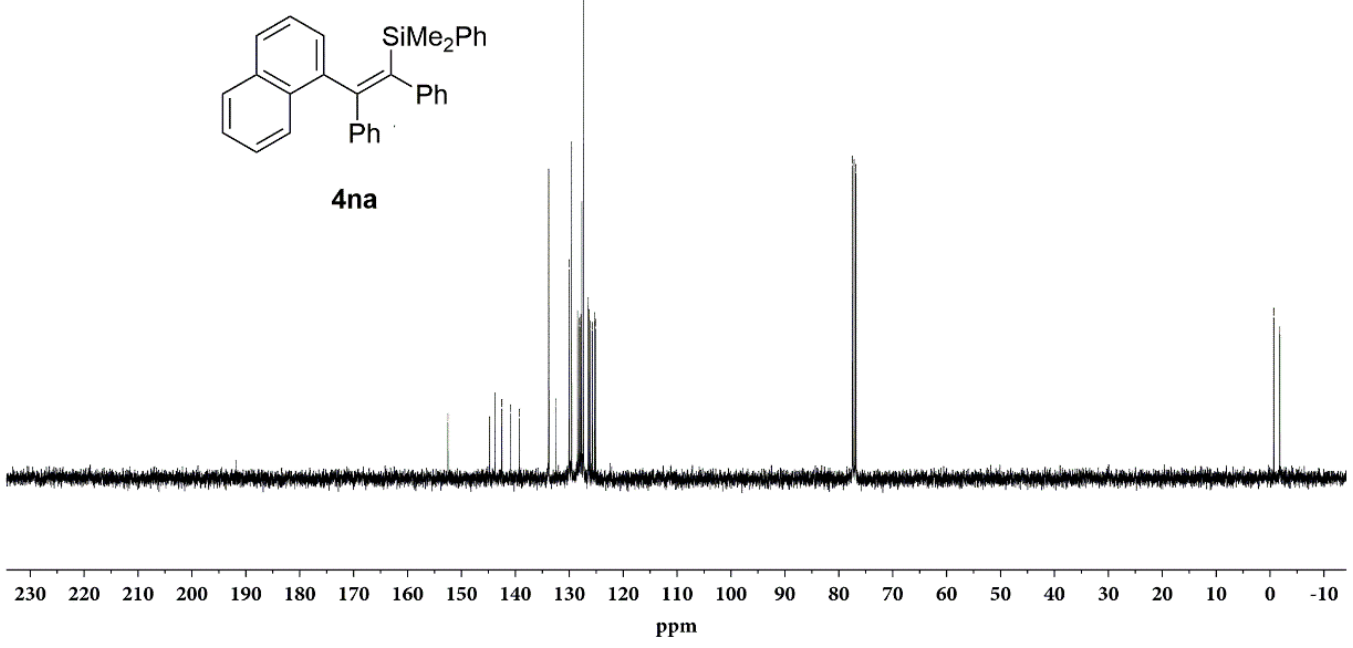

${ }^{1} \mathrm{H}$ NMR $(400 \mathrm{MHz})$ and ${ }^{13} \mathrm{C}\left\{{ }^{1} \mathrm{H}\right\}$ NMR $(101 \mathrm{MHz})$ spectra of $\mathbf{4 n a}\left(\mathrm{rt}, \mathrm{CDCl}_{3}\right)$. 


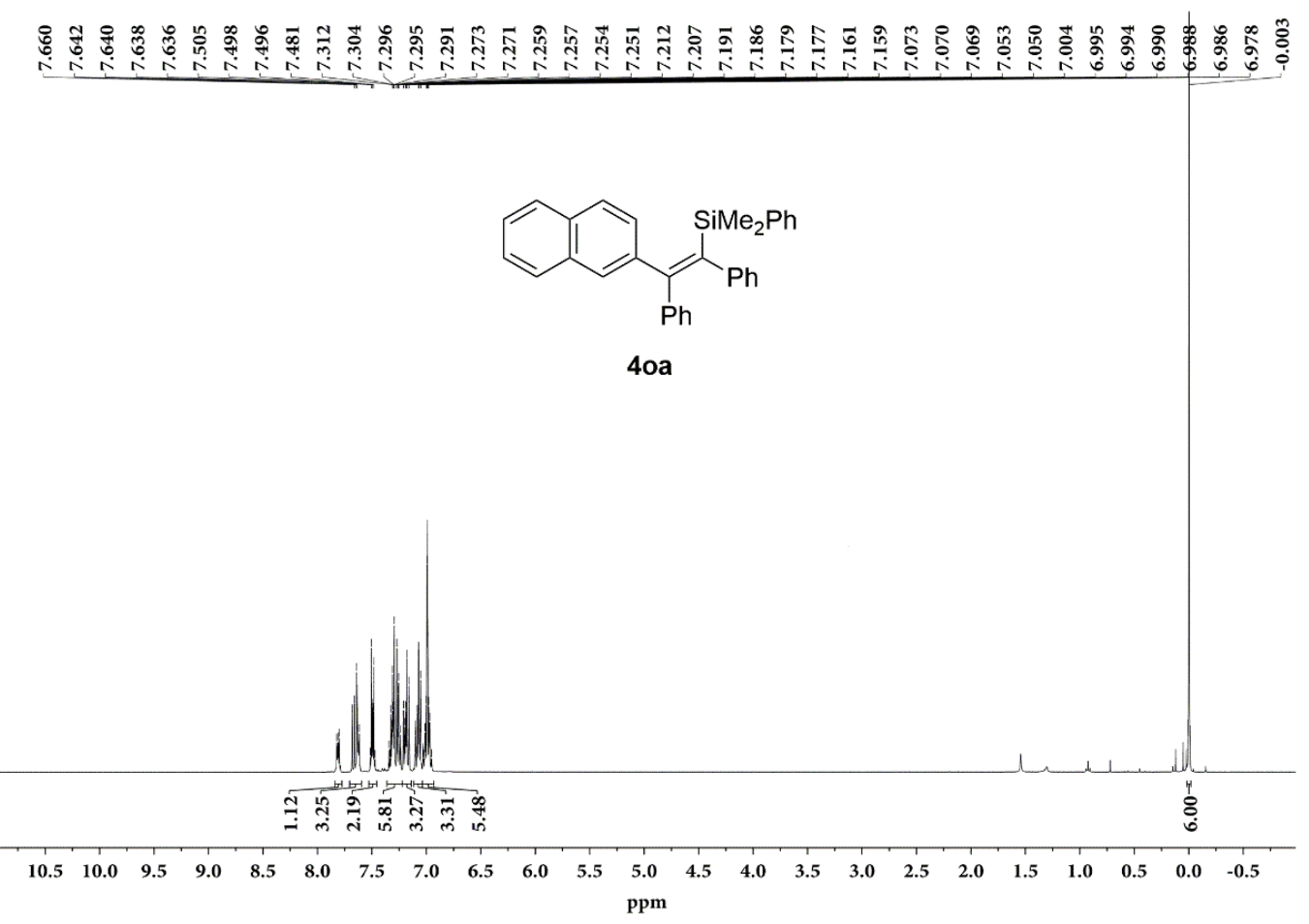

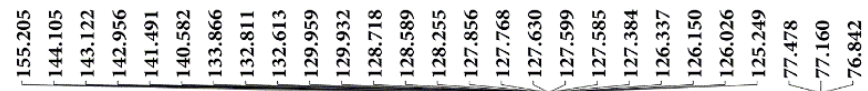

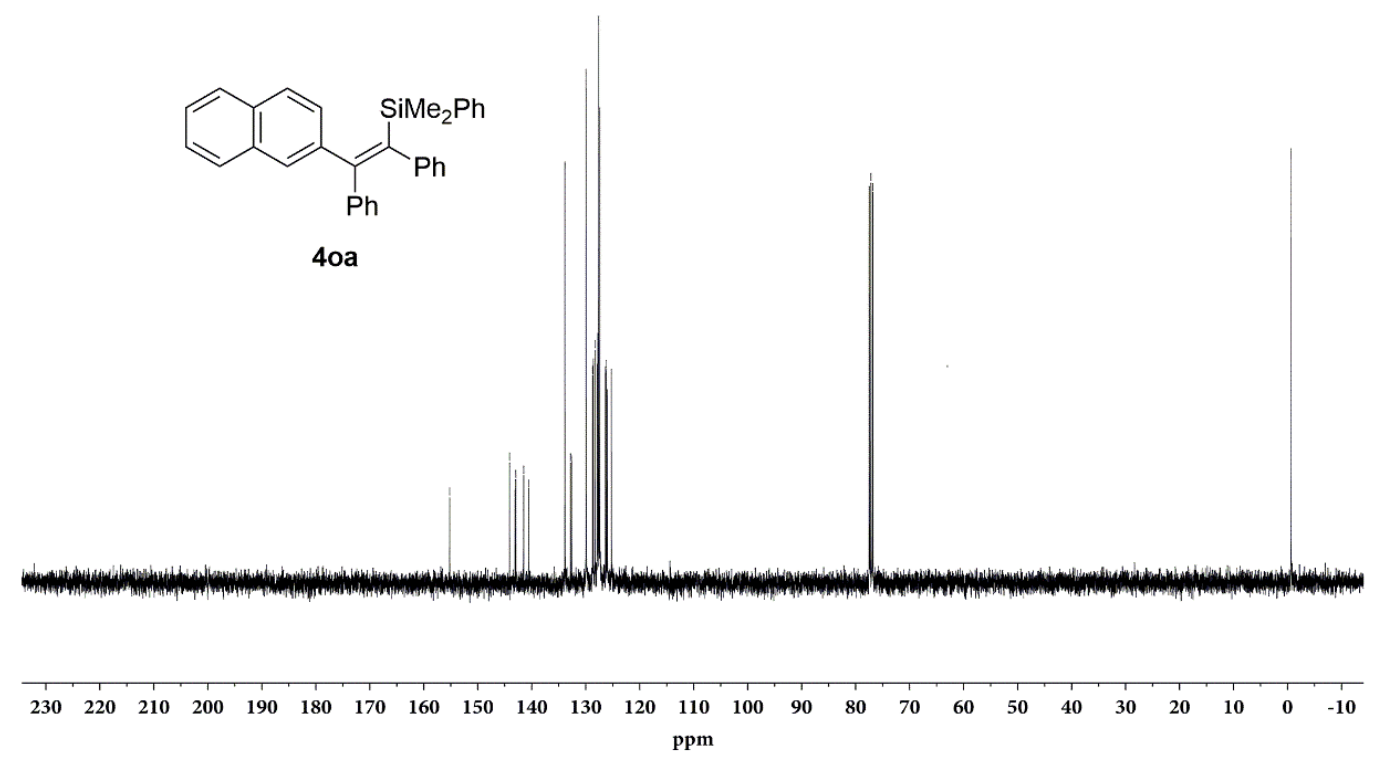

${ }^{1} \mathrm{H}$ NMR (400 MHz) and ${ }^{13} \mathrm{C}\left\{{ }^{1} \mathrm{H}\right\}$ NMR (101 MHz) spectra of $40 a\left(\mathrm{rt}, \mathrm{CDCl}_{3}\right)$. 


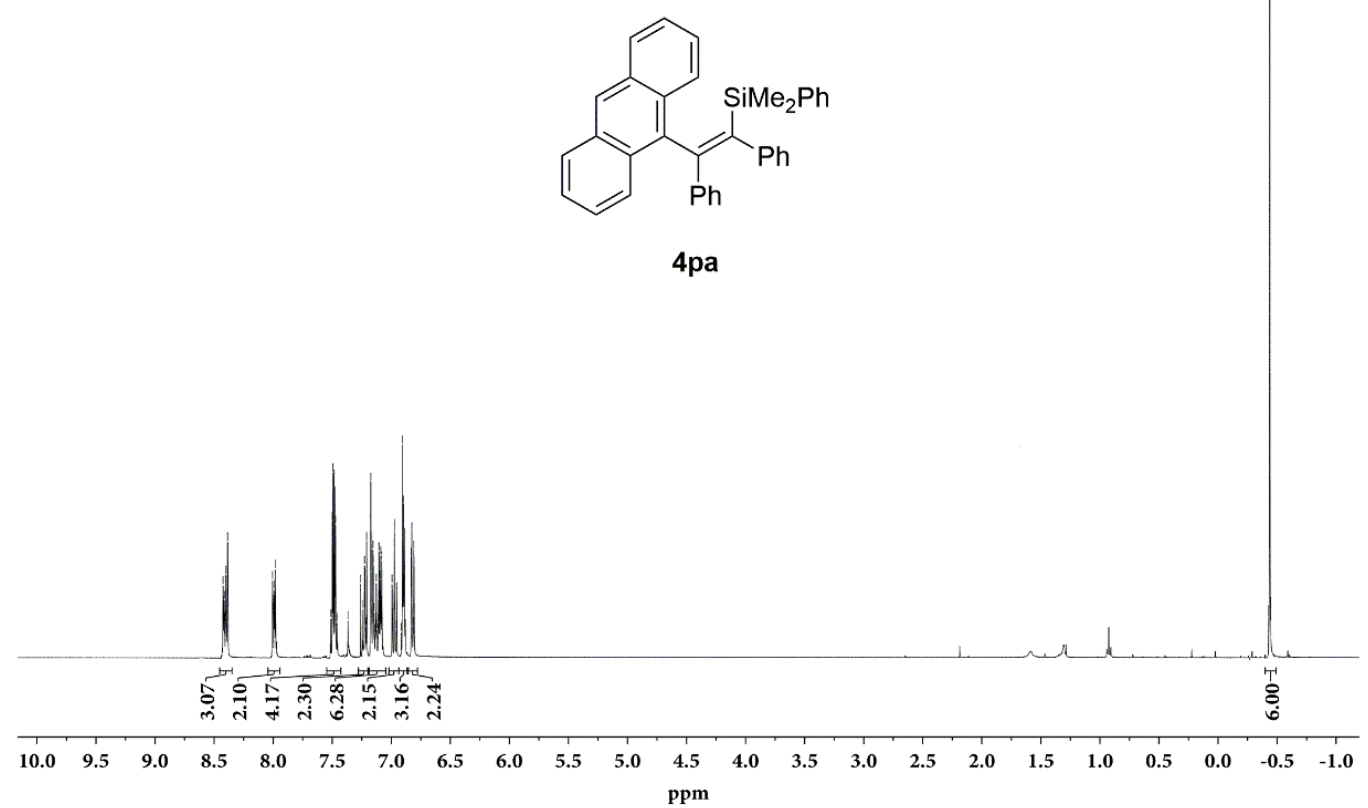

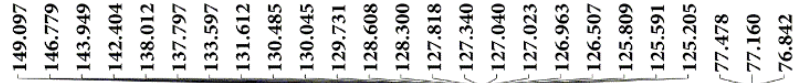

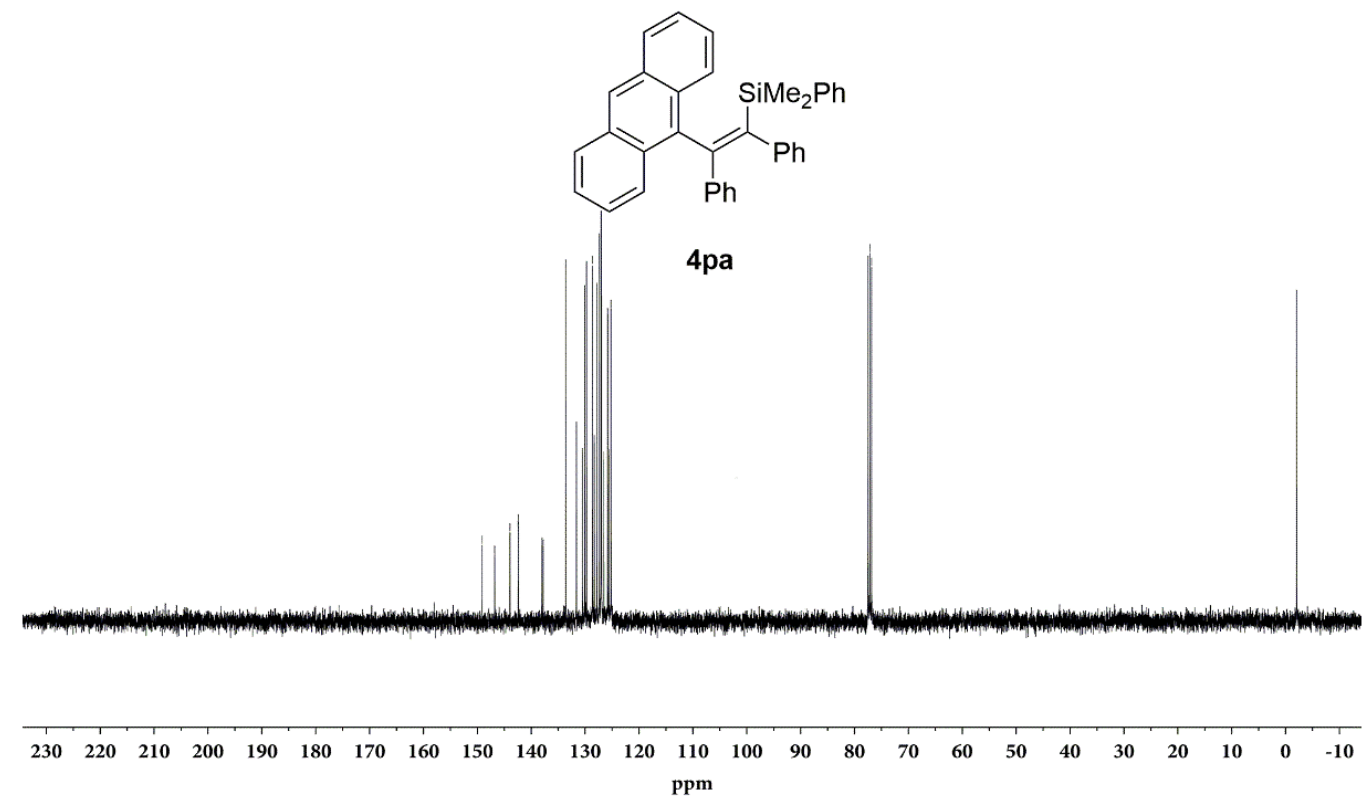

${ }^{1} \mathrm{H}$ NMR (400 MHz) and ${ }^{13} \mathrm{C}\left\{{ }^{1} \mathrm{H}\right\}$ NMR (101 MHz) spectra of 4pa (rt, $\left.\mathrm{CDCl}_{3}\right)$. 
<smiles>CC(C(=C(c1ccccc1)c1ccccc1)c1ccc2ncccc2c1)C(c1ccccc1)c1ccccc1</smiles>

4qa

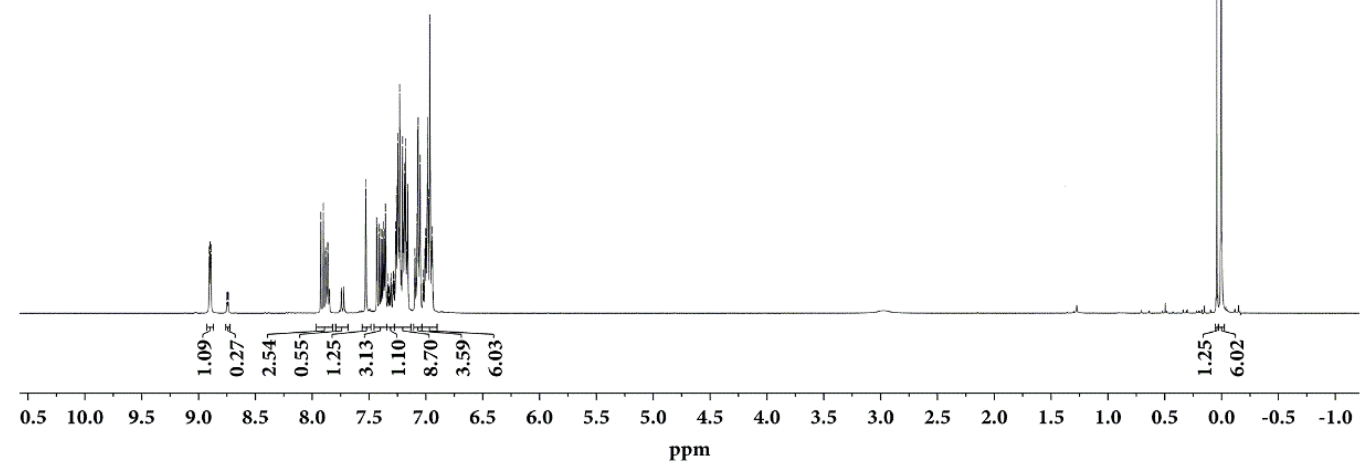

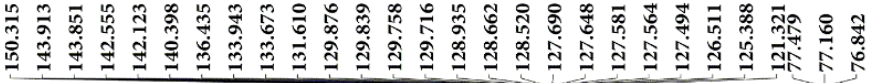<smiles>c1ccc(C(=C(c2ccccc2)[C+](c2ccccc2)c2ccccc2)c2ccccc2)cc1</smiles>

4qa

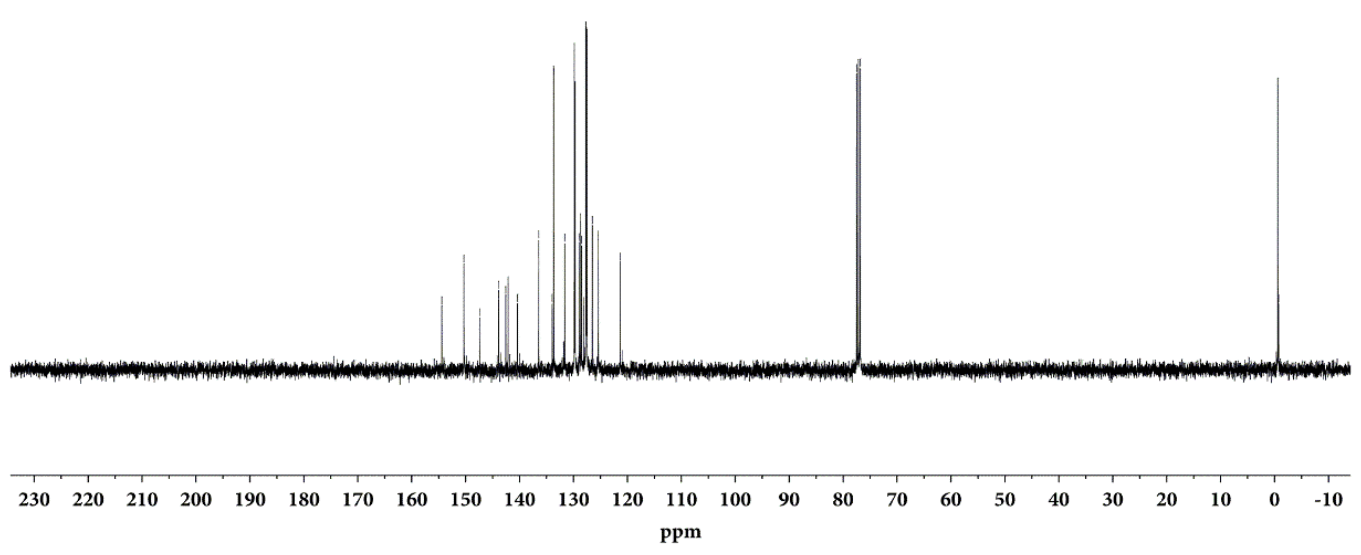

${ }^{1} \mathrm{H}$ NMR (400 MHz) and ${ }^{13} \mathrm{C}\left\{{ }^{1} \mathrm{H}\right\}$ NMR (101 MHz) spectra of $\mathbf{4 q a}\left(\mathrm{rt}, \mathrm{CDCl}_{3}\right)$. 


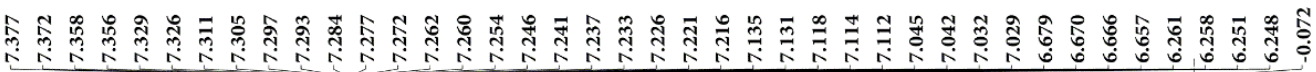

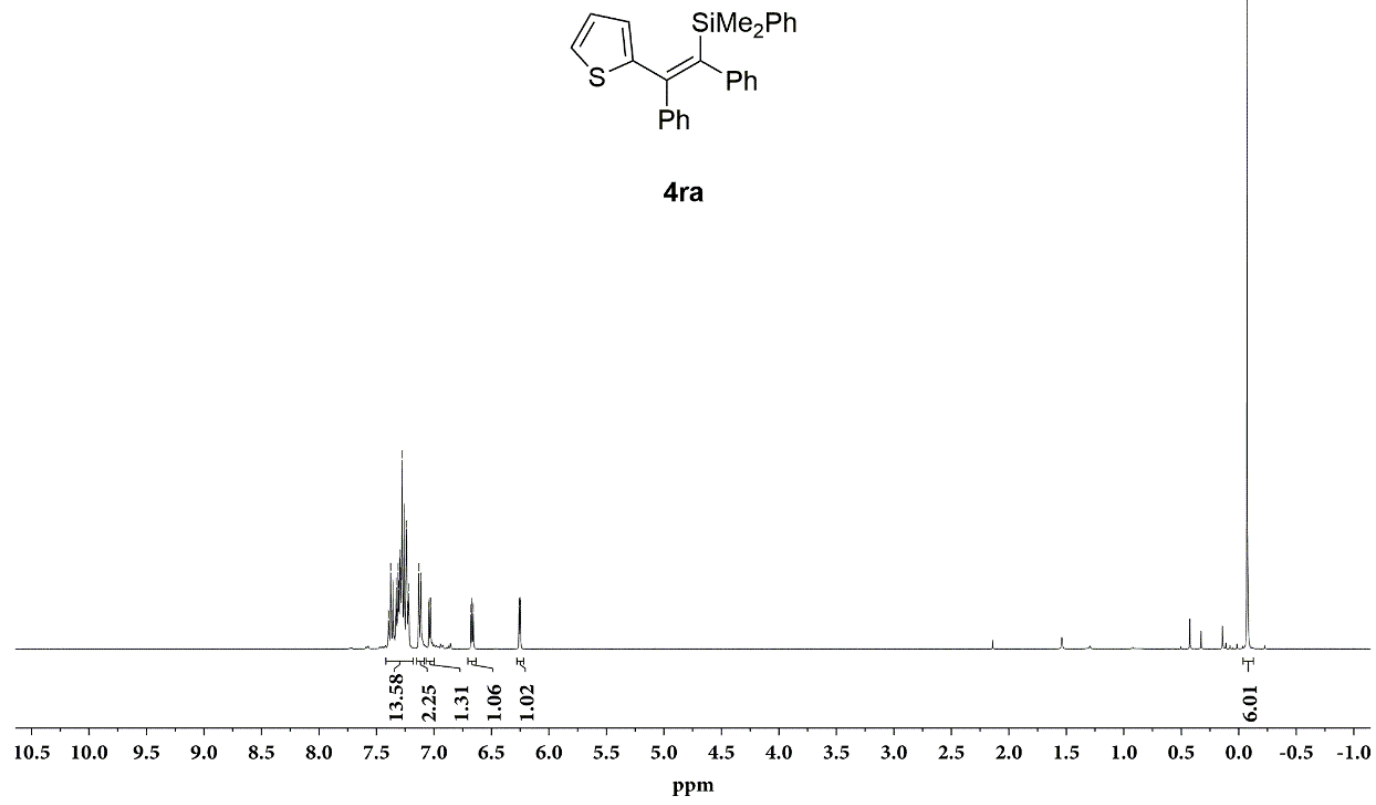

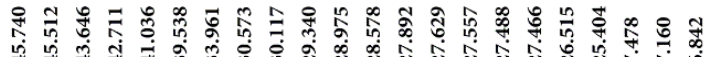

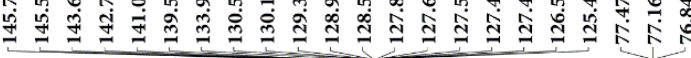
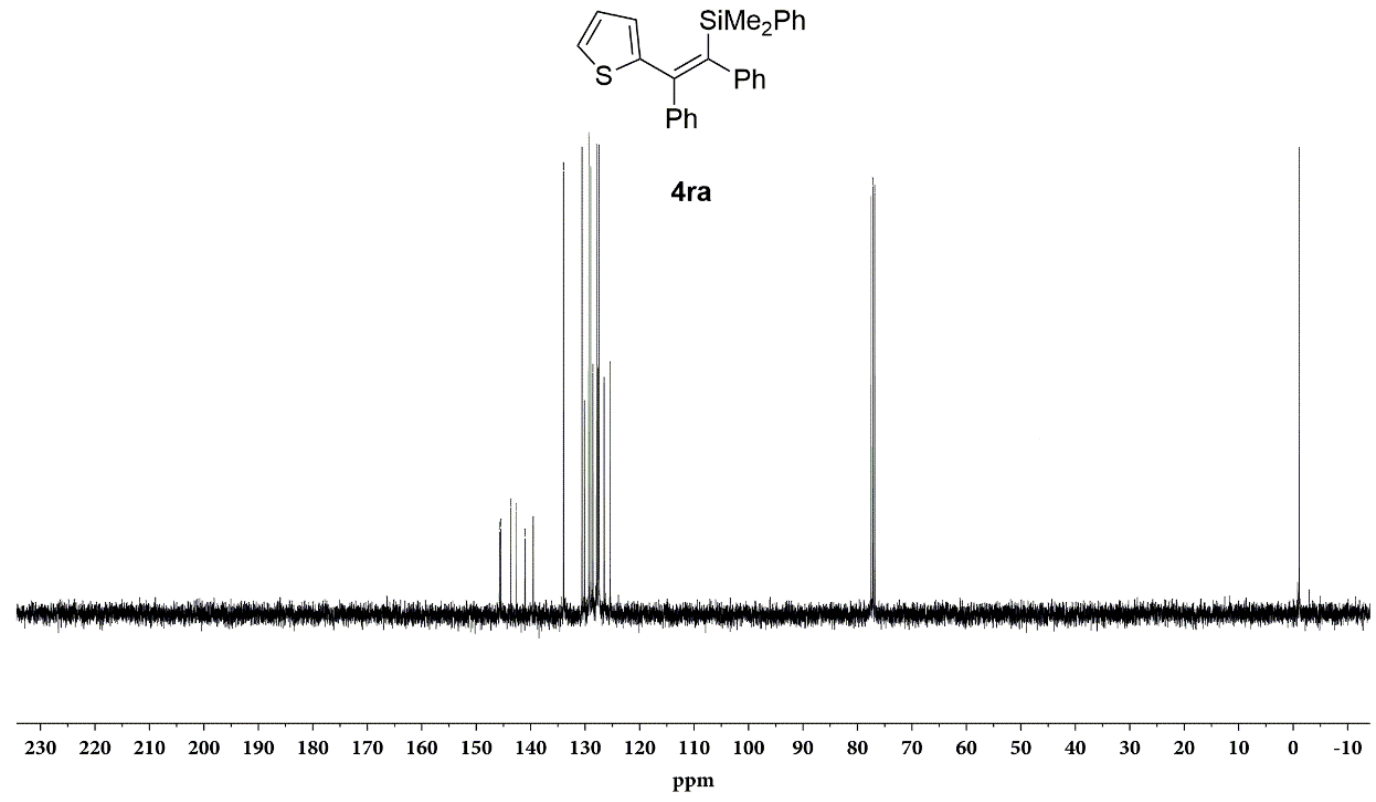

${ }^{1} \mathrm{H}$ NMR $(400 \mathrm{MHz})$ and ${ }^{13} \mathrm{C}\left\{{ }^{1} \mathrm{H}\right\}$ NMR $(101 \mathrm{MHz})$ spectra of 4 ra $\left(\mathrm{rt}, \mathrm{CDCl}_{3}\right)$. 


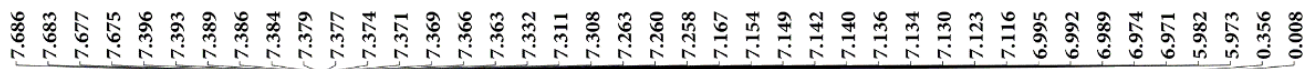

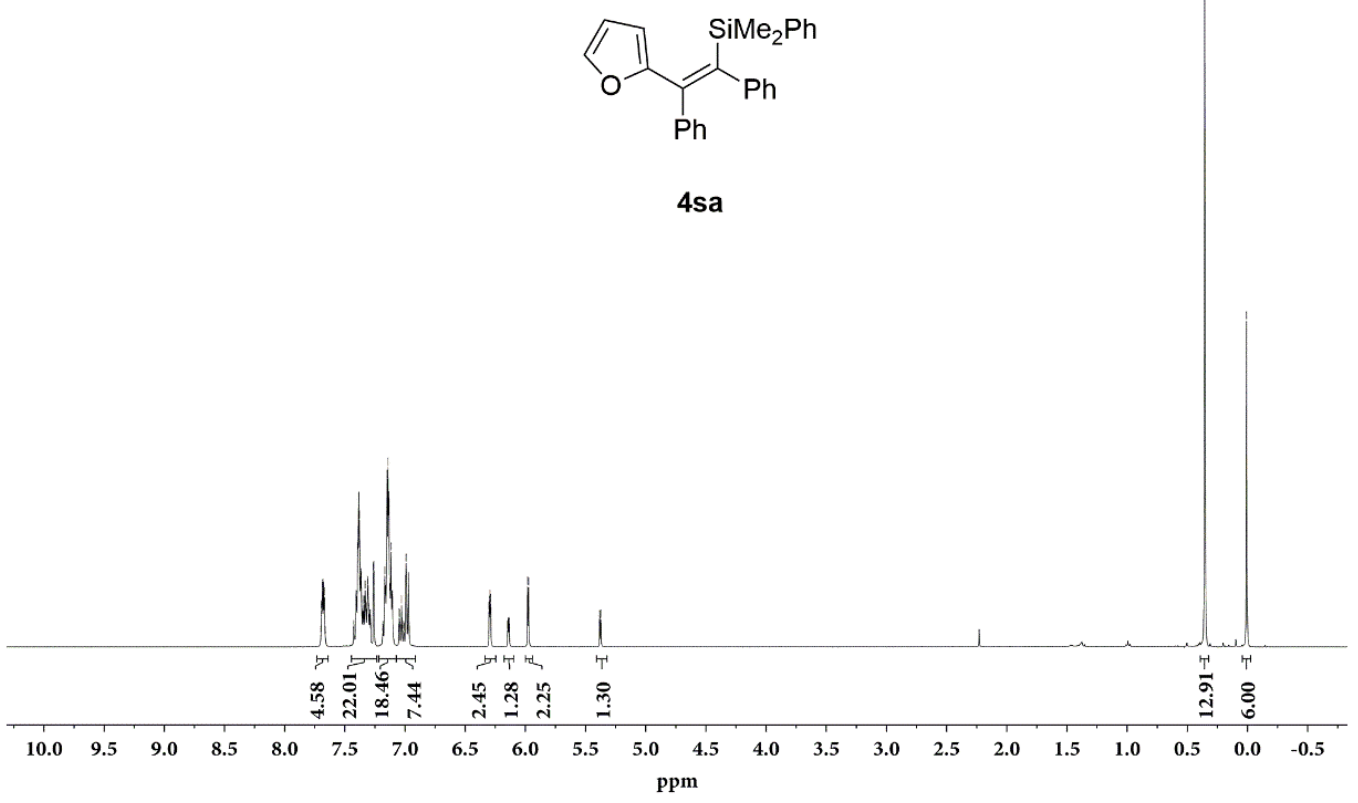

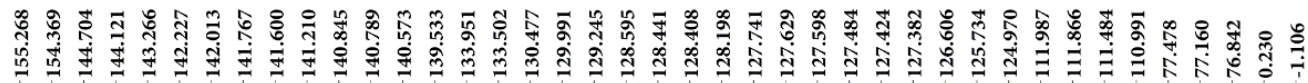

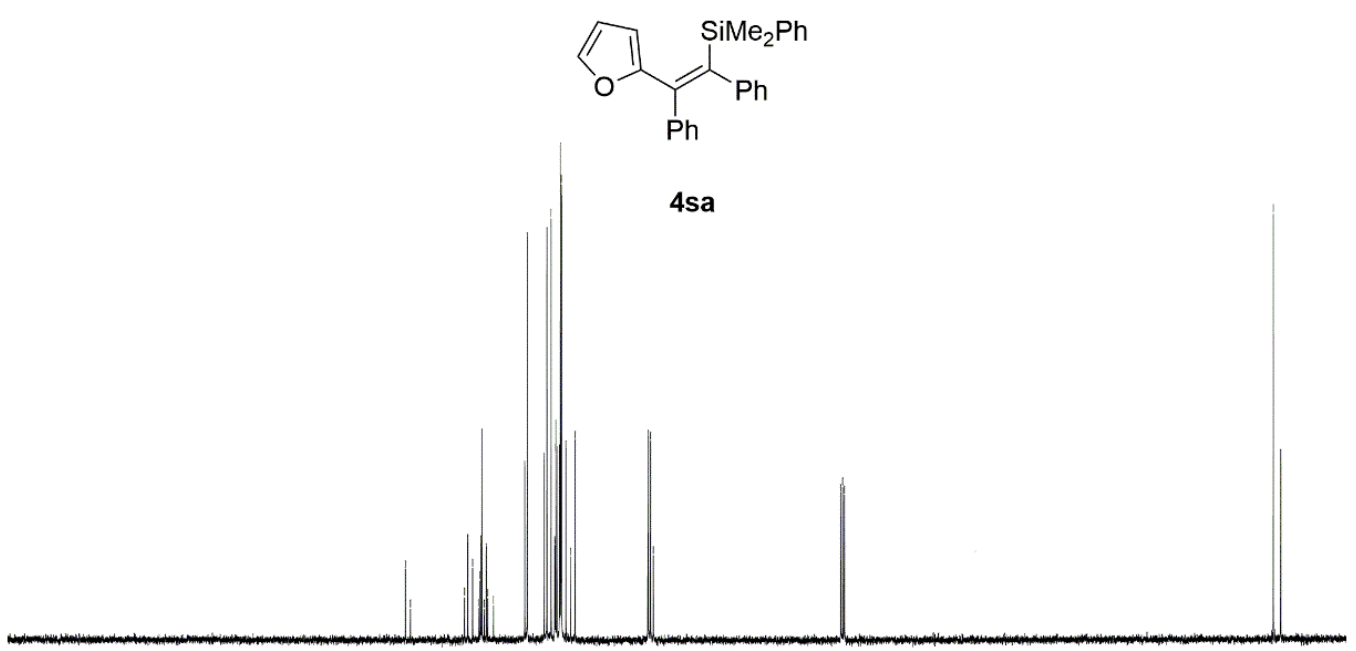

$\begin{array}{lllllllllllllllllllllllllllll}220 & 210 & 200 & 190 & 180 & 170 & 160 & 150 & 140 & 130 & 120 & 110 & 100 & 90 & 80 & 70 & 60 & 50 & 40 & 30 & 20 & 10 & 0 & -10\end{array}$

${ }^{1} \mathrm{H}$ NMR (400 MHz) and ${ }^{13} \mathrm{C}\left\{{ }^{1} \mathrm{H}\right\}$ NMR $(101 \mathrm{MHz})$ spectra of $4 \mathbf{s a}\left(\mathrm{rt}, \mathrm{CDCl}_{3}\right)$. 
${ }^{1} \mathrm{H}$ NMR (400 MHz), ${ }^{13} \mathrm{C}\left\{{ }^{1} \mathrm{H}\right\}$ NMR (101 MHz), and ${ }^{19} \mathrm{~F}\left\{{ }^{1} \mathrm{H}\right\}$ NMR (376 MHz) spectra of 3ia (rt, $\mathrm{CDCl}_{3}$

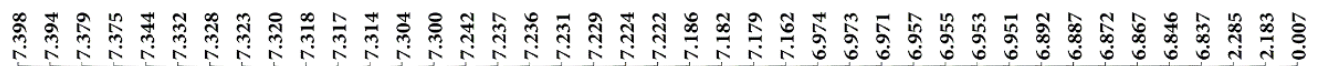
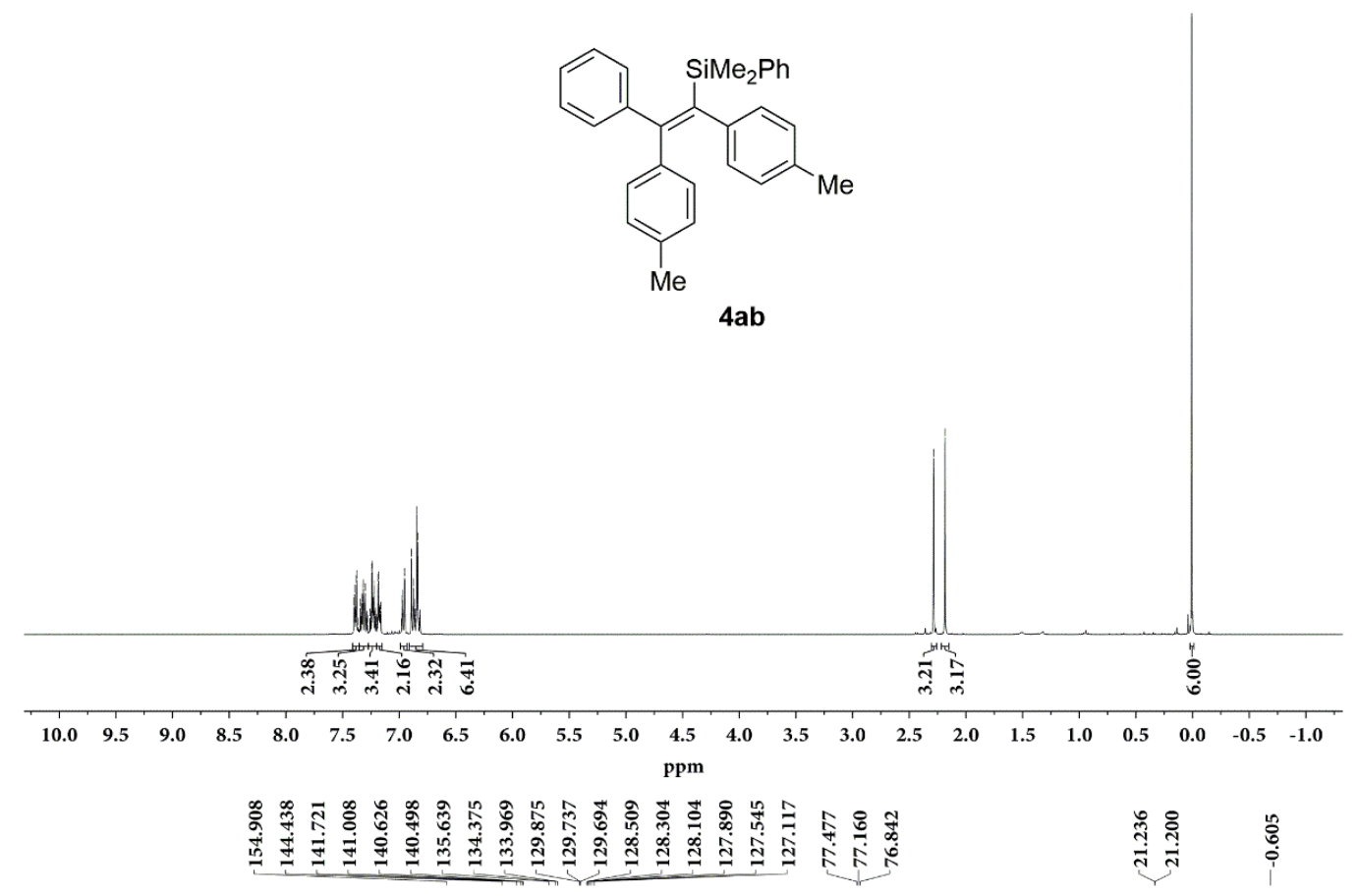

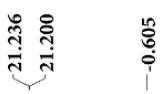

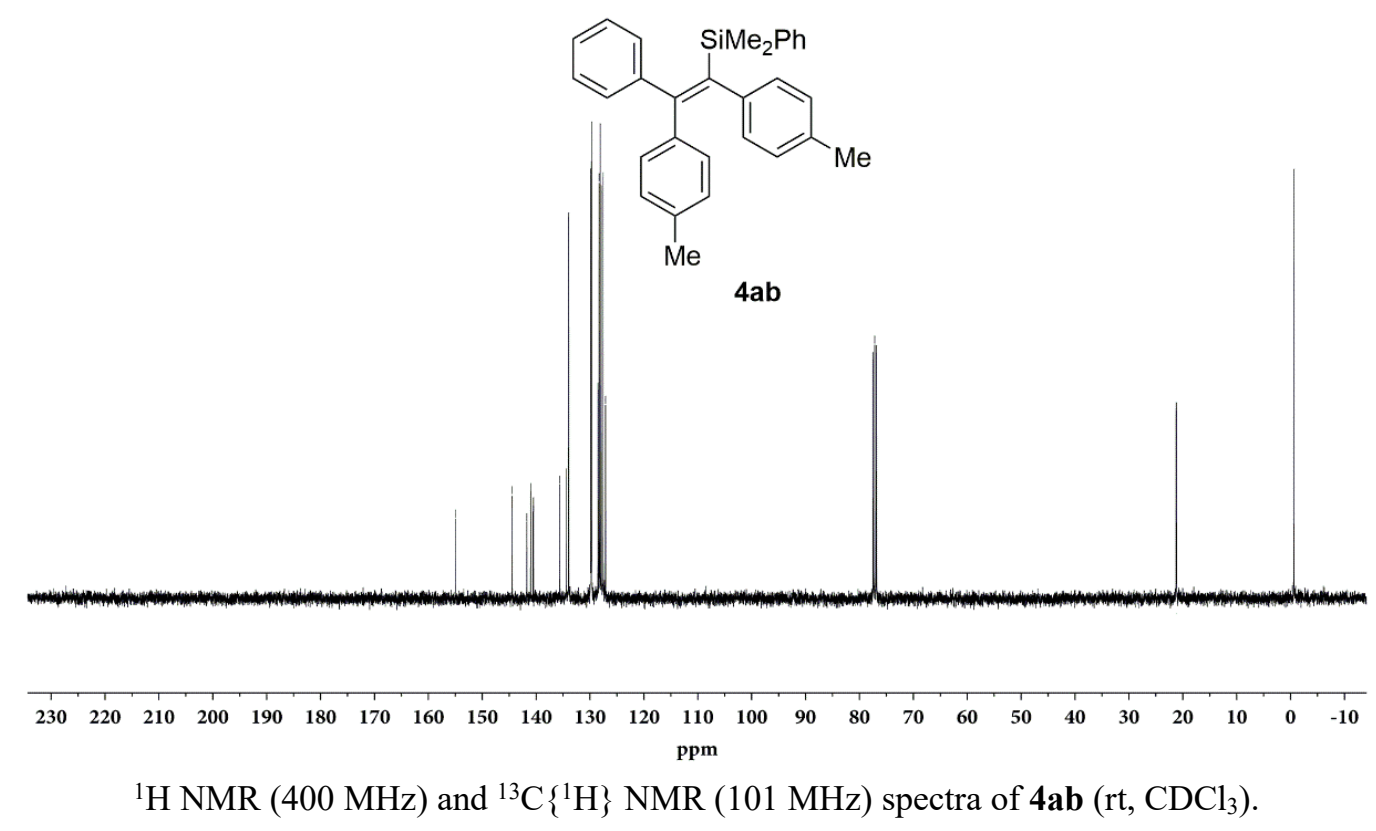




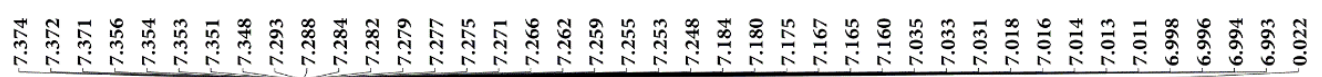

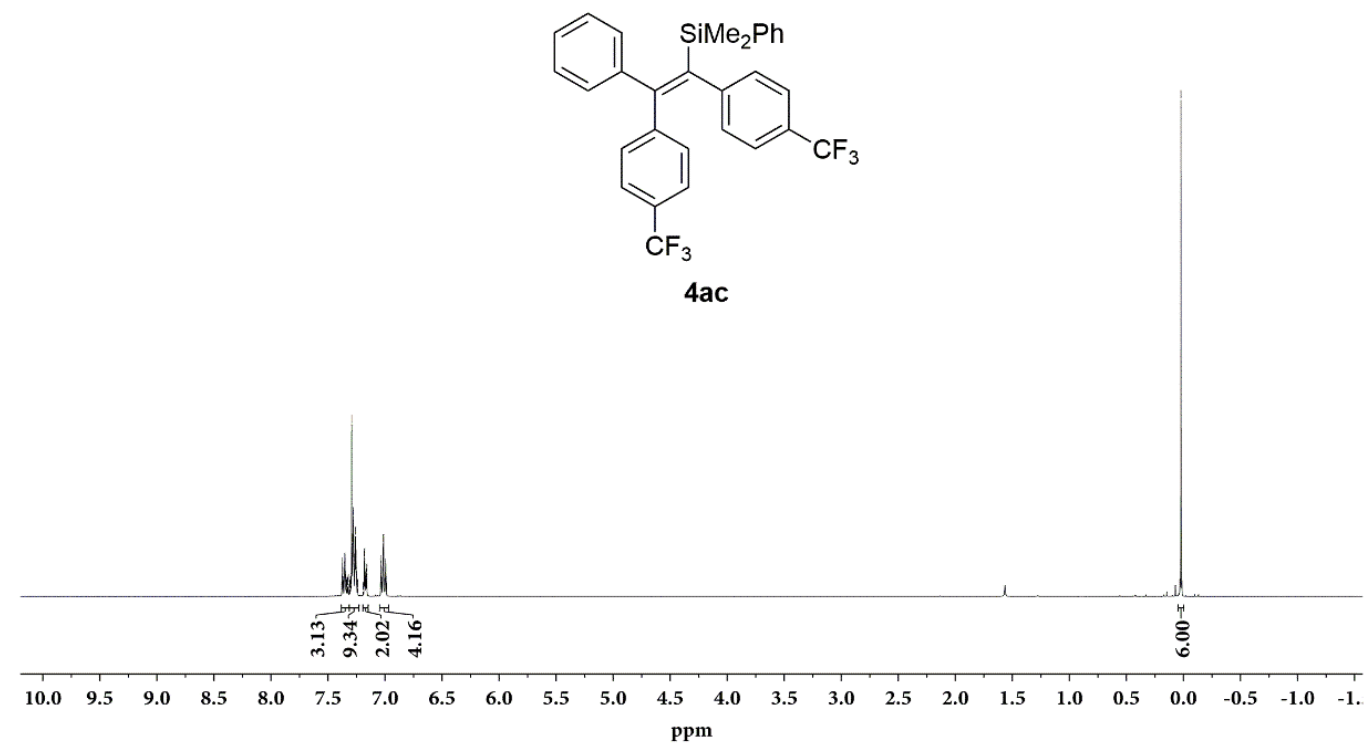

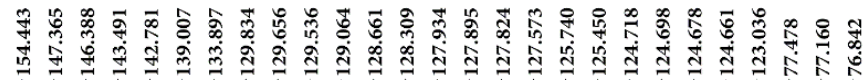

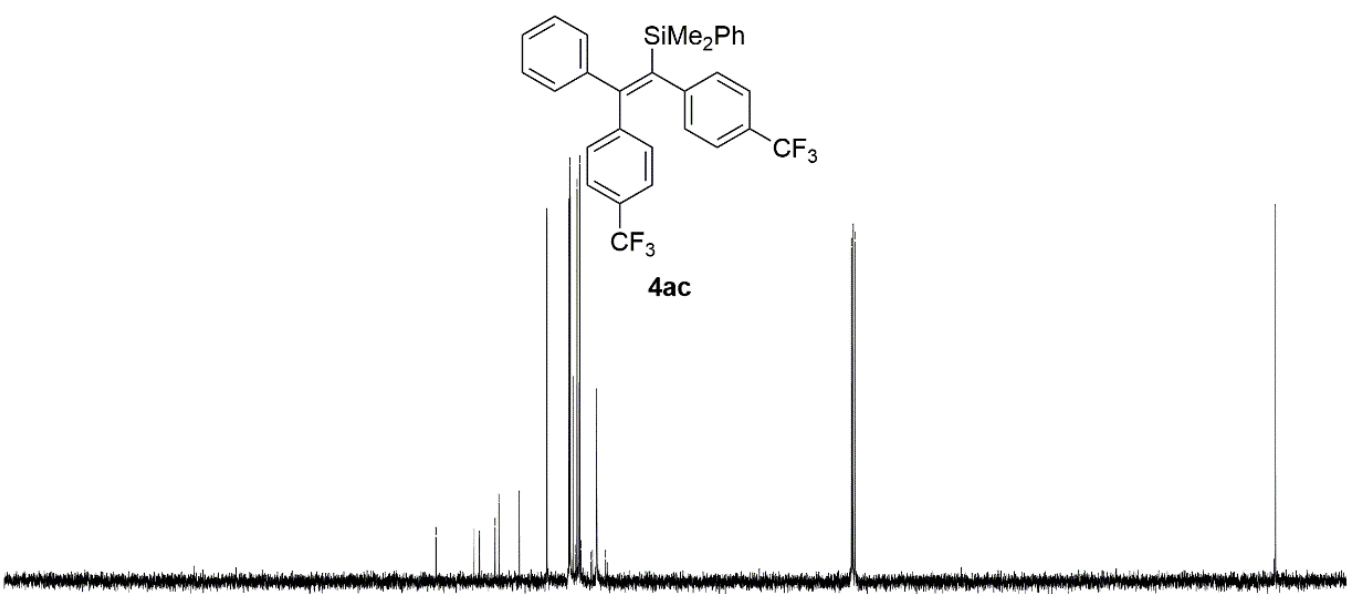

$\begin{array}{llllllllllllllllllllllllllllllllllll}230 & 220 & 210 & 200 & 190 & 180 & 170 & 160 & 150 & 140 & 130 & 120 & 110 & 100 & 90 & 80 & 70 & 60 & 50 & 40 & 30 & 20 & 10 & 0 & -10\end{array}$

${ }^{1} \mathrm{H}$ NMR $(400 \mathrm{MHz})$ and ${ }^{13} \mathrm{C}\left\{{ }^{1} \mathrm{H}\right\}$ NMR (101 MHz) spectra of $\mathbf{4 a c}\left(\mathrm{rt}, \mathrm{CDCl}_{3}\right)$. 


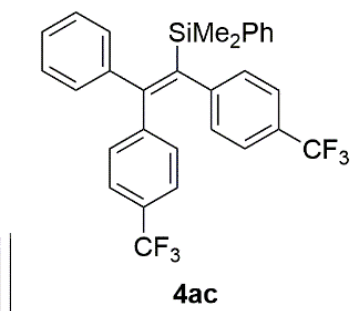

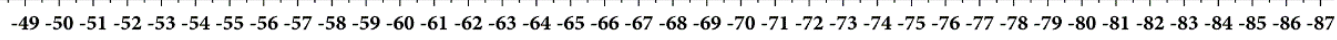
ppm

${ }^{19} \mathrm{~F}\left\{{ }^{1} \mathrm{H}\right\}$ NMR (376 MHz) spectrum of $4 \mathbf{a c}\left(\mathrm{rt}, \mathrm{CDCl}_{3}\right)$. 


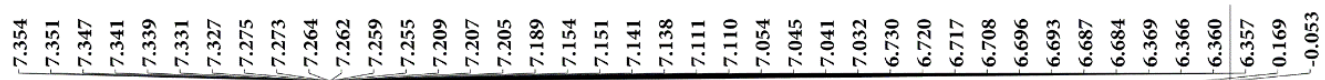
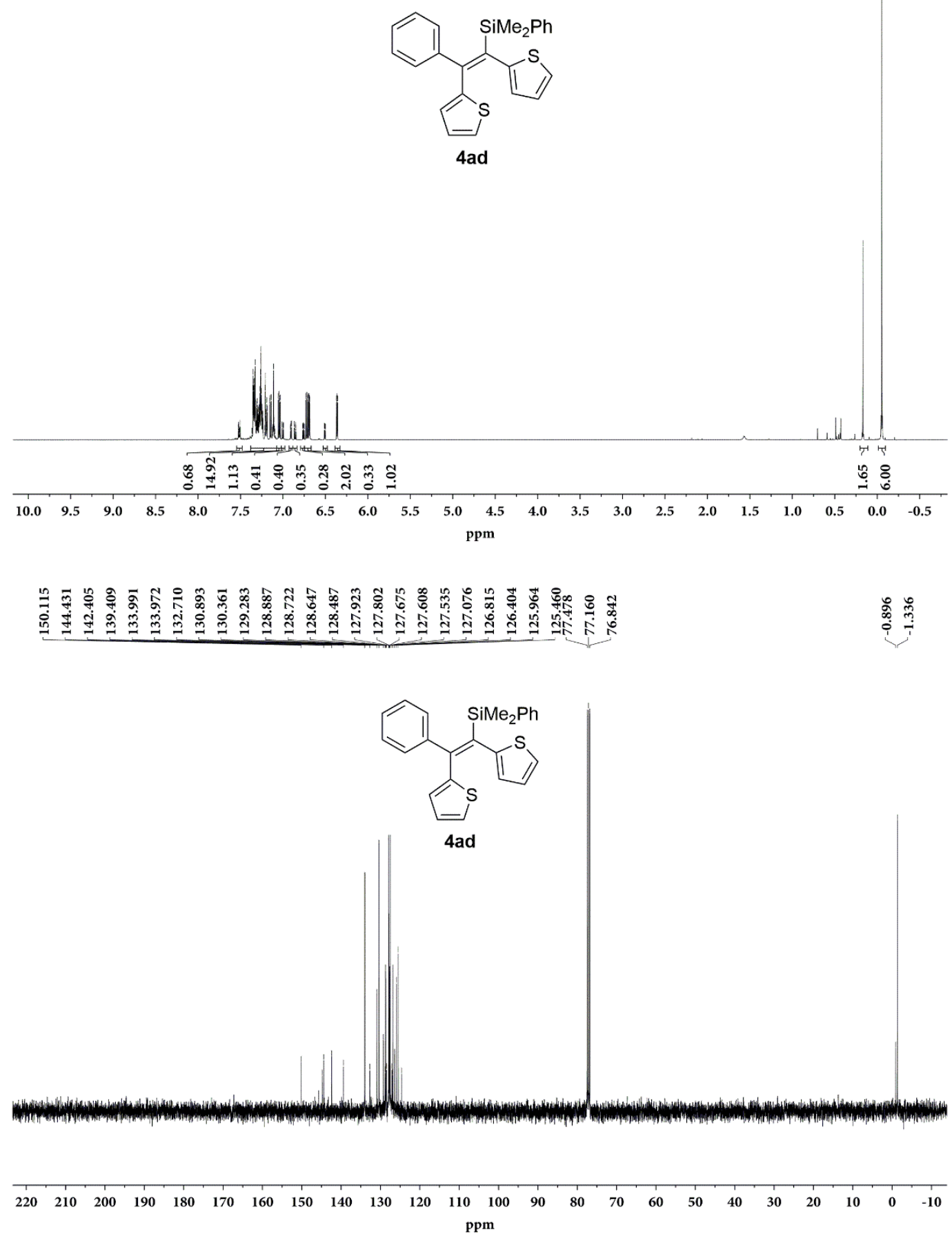

${ }^{1} \mathrm{H}$ NMR $(400 \mathrm{MHz})$ and ${ }^{13} \mathrm{C}\left\{{ }^{1} \mathrm{H}\right\}$ NMR $(101 \mathrm{MHz})$ spectra of $\mathbf{4 a d}\left(\mathrm{rt}, \mathrm{CDCl}_{3}\right)$. 


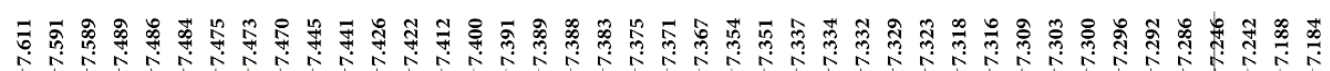

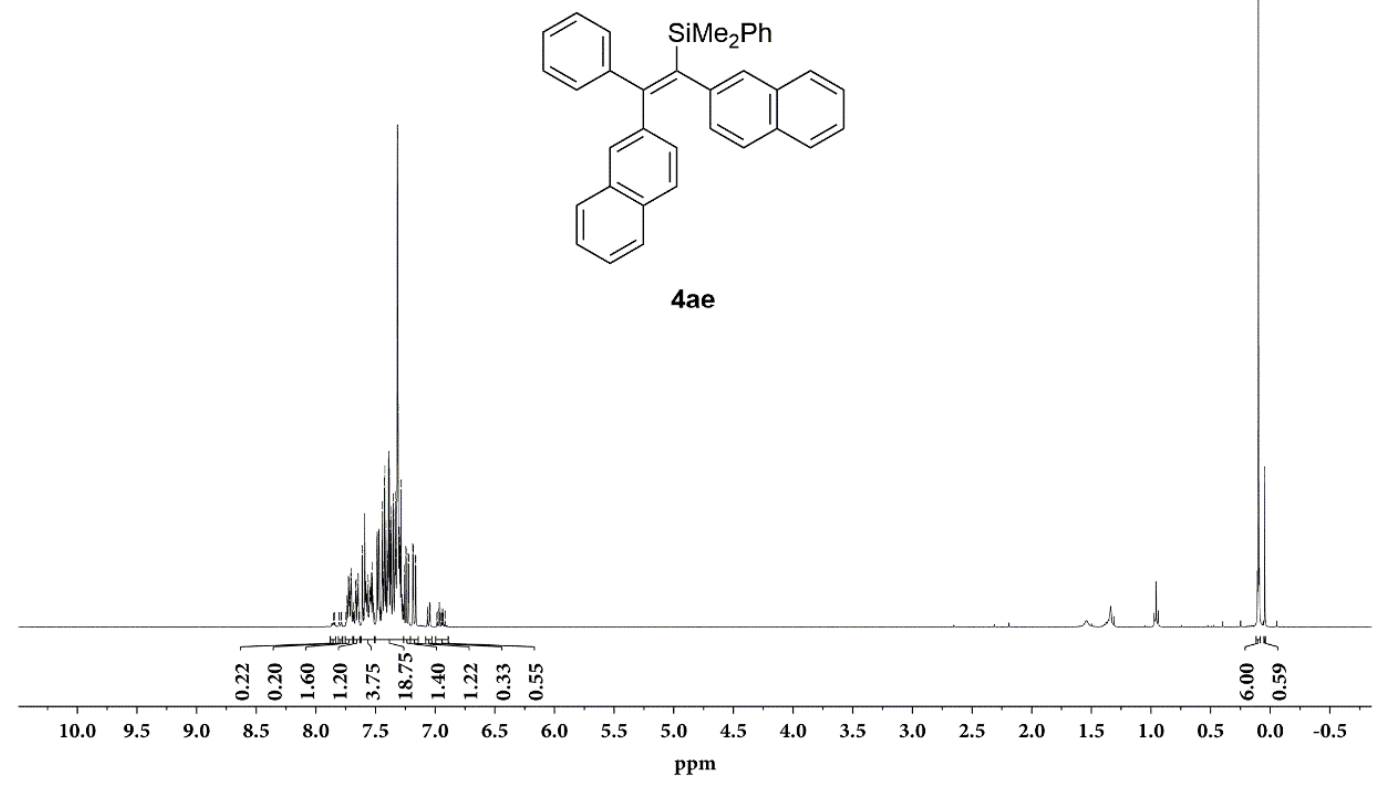

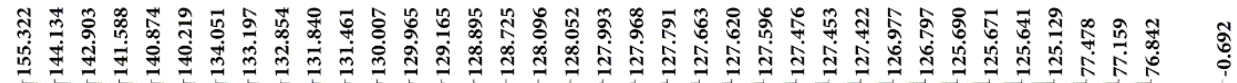

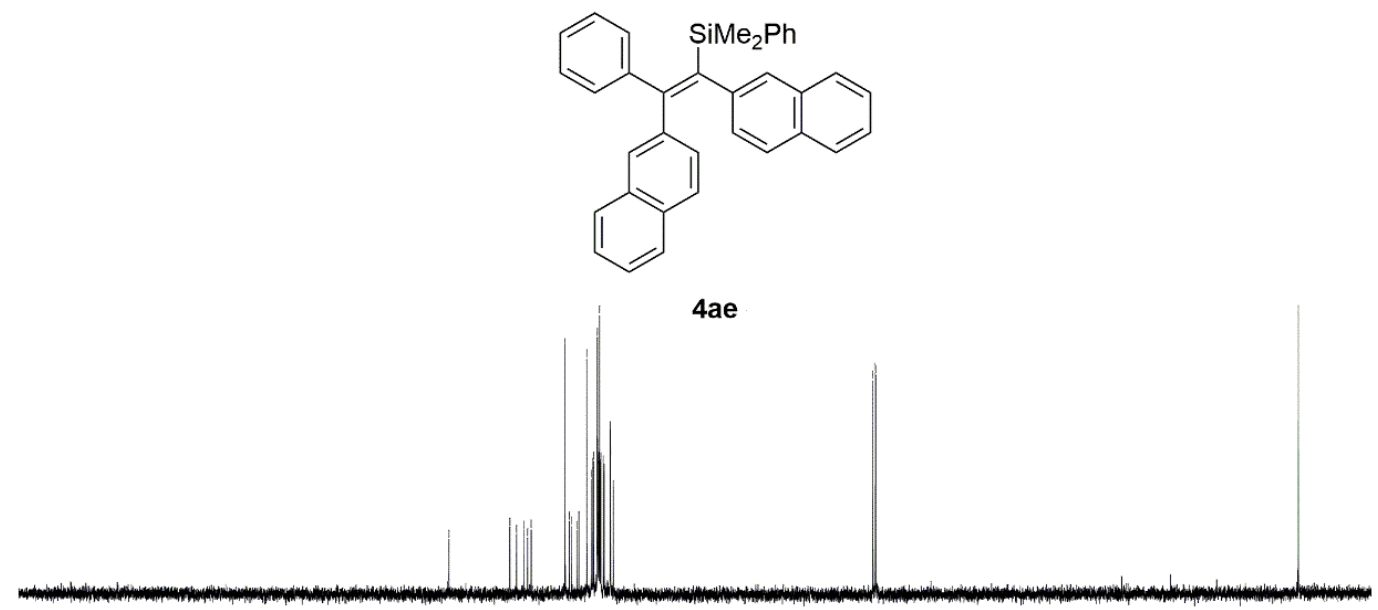

$\begin{array}{llllllllllllllllllllllllll}230 & 220 & 210 & 200 & 190 & 180 & 170 & 160 & 150 & 140 & 130 & 120 & 110 & 100 & 90 & 80 & 70 & 60 & 50 & 40 & 30 & 20 & 10 & 0 & -10\end{array}$ ppm

${ }^{1} \mathrm{H}$ NMR $(400 \mathrm{MHz})$ and ${ }^{13} \mathrm{C}\left\{{ }^{1} \mathrm{H}\right\}$ NMR (101 MHz) spectra of 4ae (rt, $\left.\mathrm{CDCl}_{3}\right)$. 


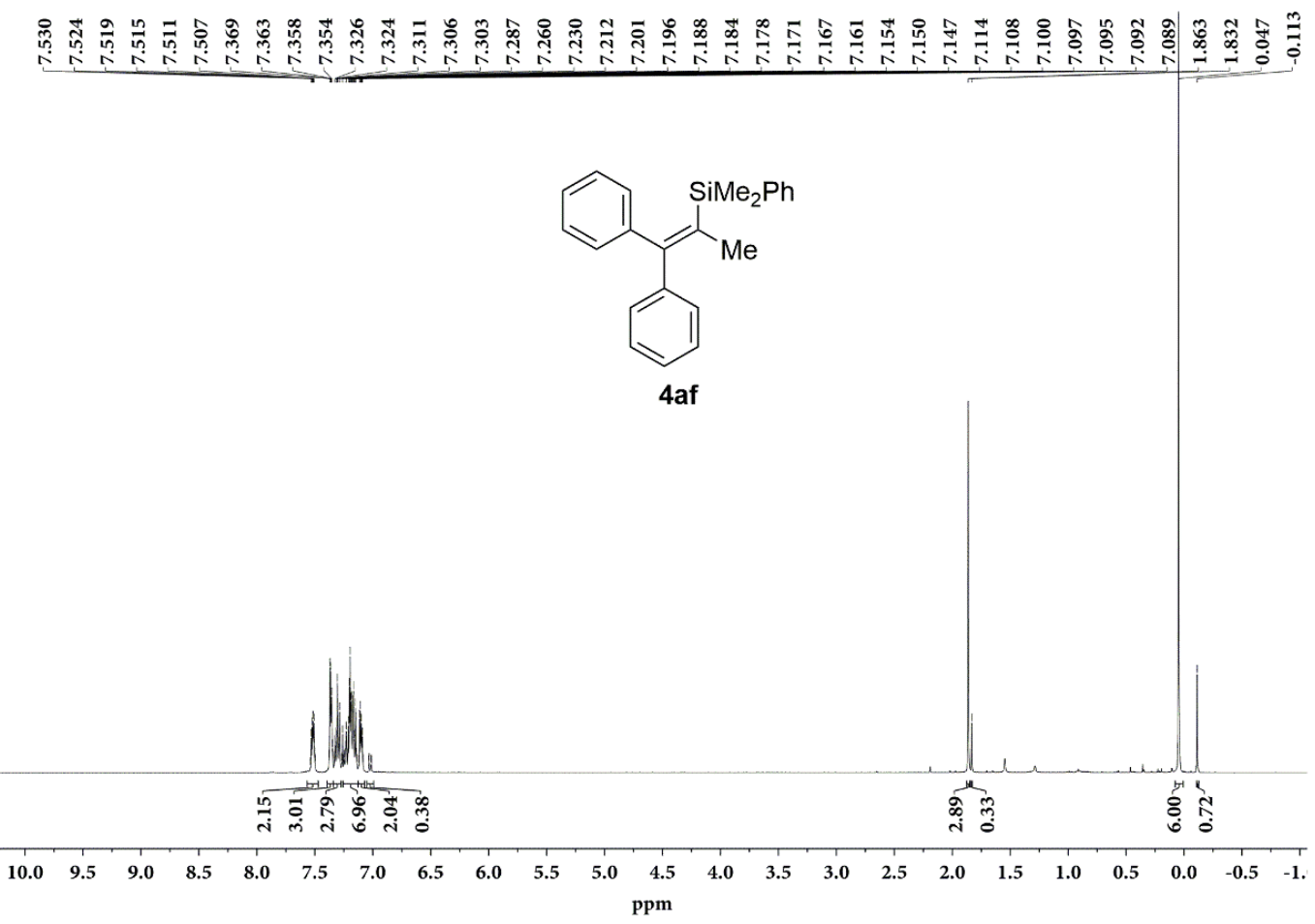

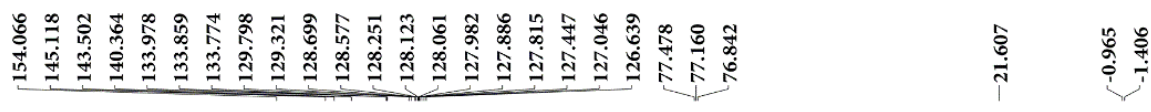<smiles>O=[N+]([O-])C(c1ccccc1)c1ccccc1</smiles>

4af

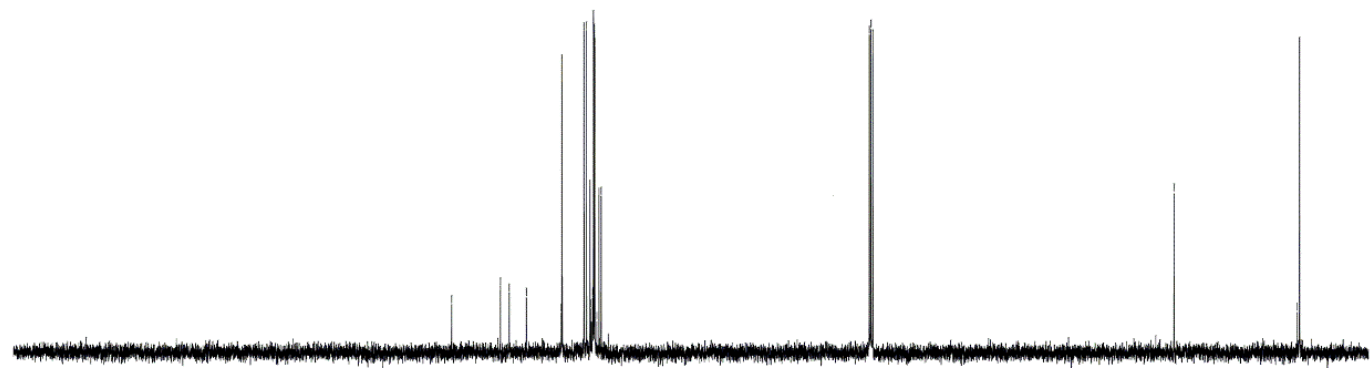

$\begin{array}{lllllllllllllllllllllllll}230 & 220 & 210 & 200 & 190 & 180 & 170 & 160 & 150 & 140 & 130 & 120 & 110 & 100 & 90 & 80 & 70 & 60 & 50 & 40 & 30 & 20 & 10 & 0 & -10\end{array}$ ppm

${ }^{1} \mathrm{H}$ NMR (400 MHz) and ${ }^{13} \mathrm{C}\left\{{ }^{1} \mathrm{H}\right\}$ NMR (101 MHz) spectra of $\mathbf{4 a f}\left(\mathrm{rt}, \mathrm{CDCl}_{3}\right)$. 

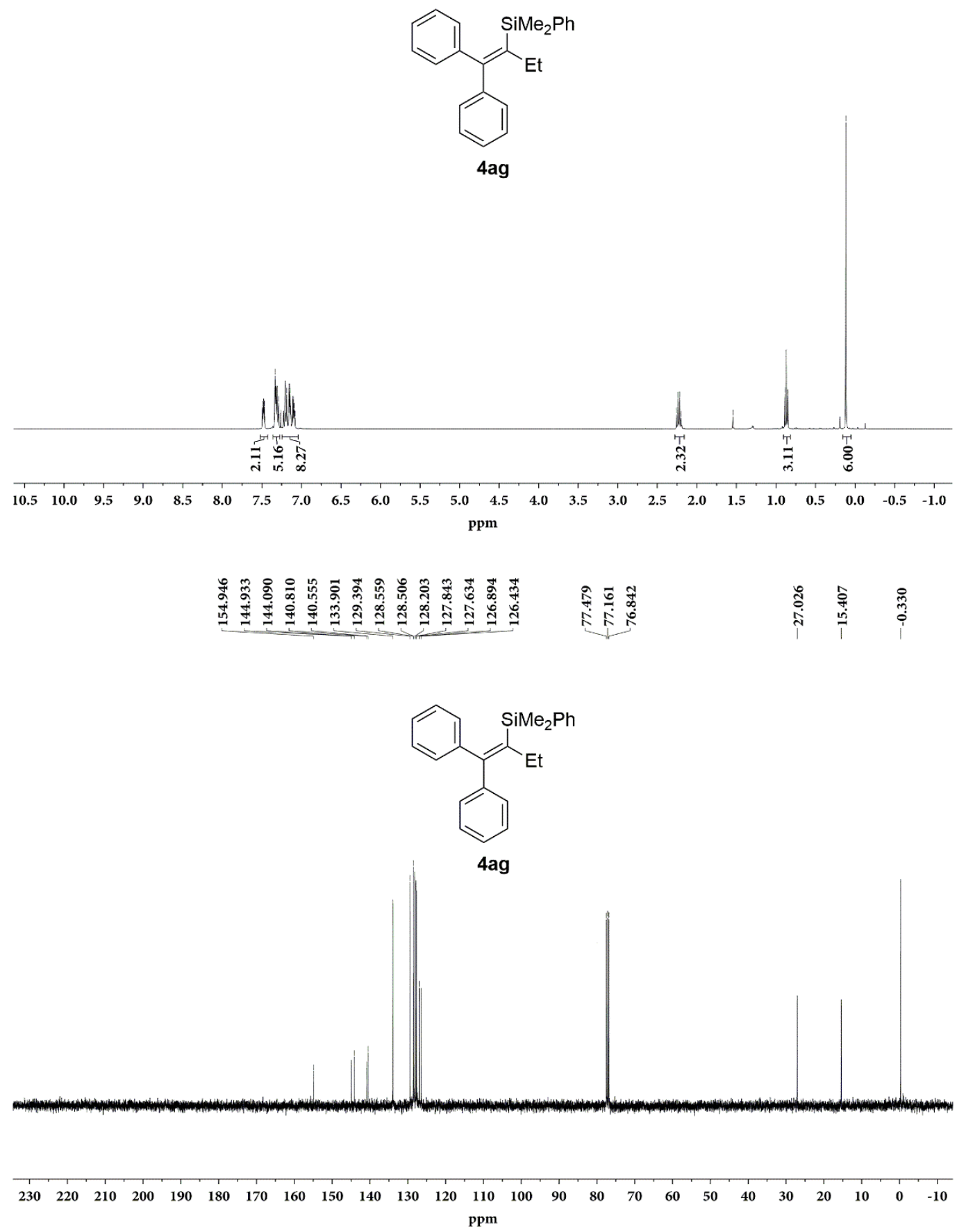

${ }^{1} \mathrm{H}$ NMR (400 MHz) and ${ }^{13} \mathrm{C}\left\{{ }^{1} \mathrm{H}\right\}$ NMR (101 MHz) spectra of 4ag (rt, $\left.\mathrm{CDCl}_{3}\right)$. 

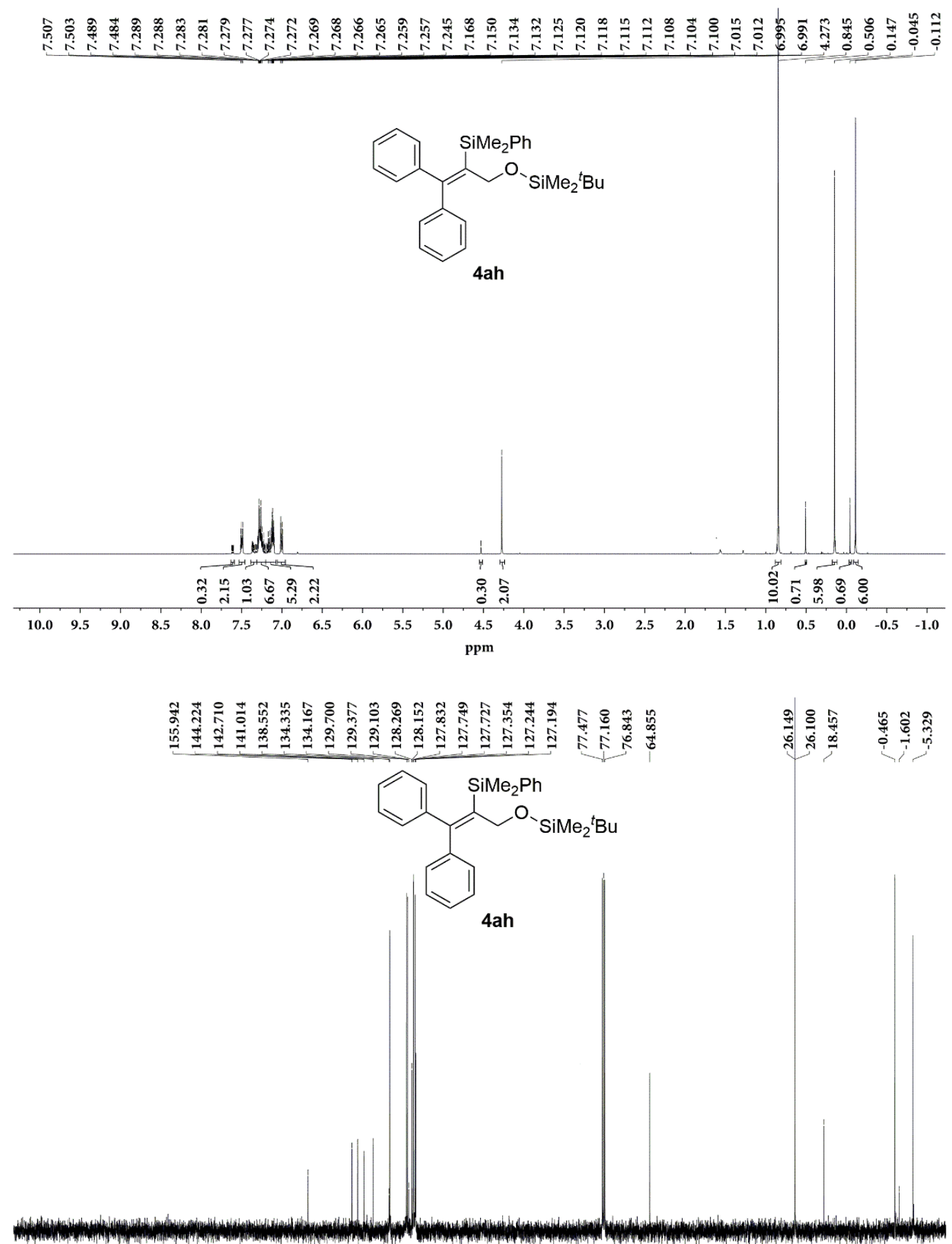

$\begin{array}{llllllllllllllllllllllllll}230 & 220 & 210 & 200 & 190 & 180 & 170 & 160 & 150 & 140 & 130 & 120 & 110 & 100 & 90 & 80 & 70 & 60 & 50 & 40 & 30 & 20 & 10 & 0 & -10\end{array}$ ppm

${ }^{1} \mathrm{H}$ NMR (400 MHz) and ${ }^{13} \mathrm{C}\left\{{ }^{1} \mathrm{H}\right\}$ NMR (101 MHz) spectra of 4ah (rt, $\left.\mathrm{CDCl}_{3}\right)$. 


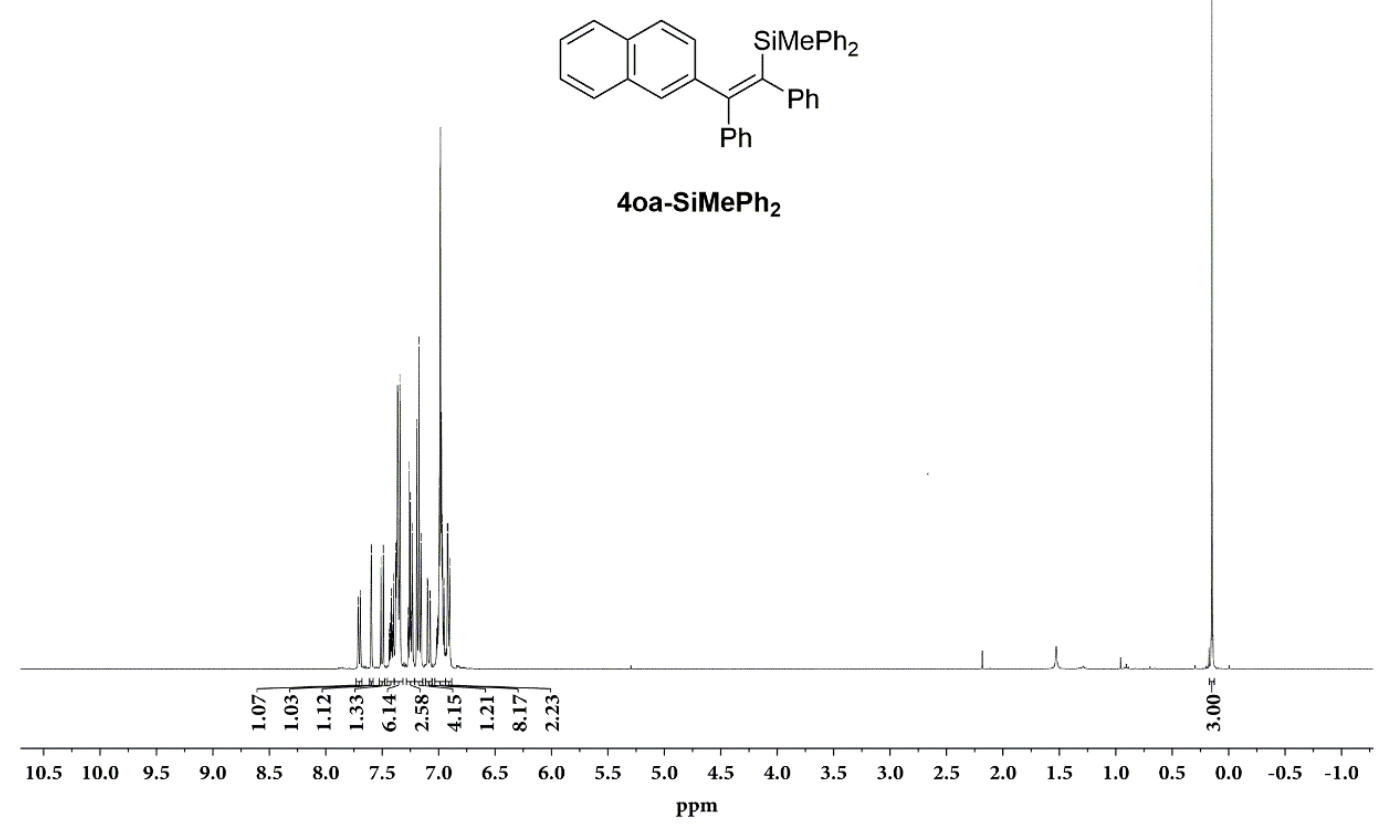

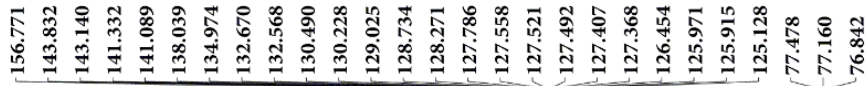

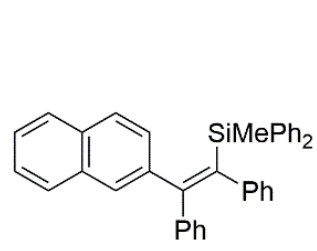

4oa-SiMePh

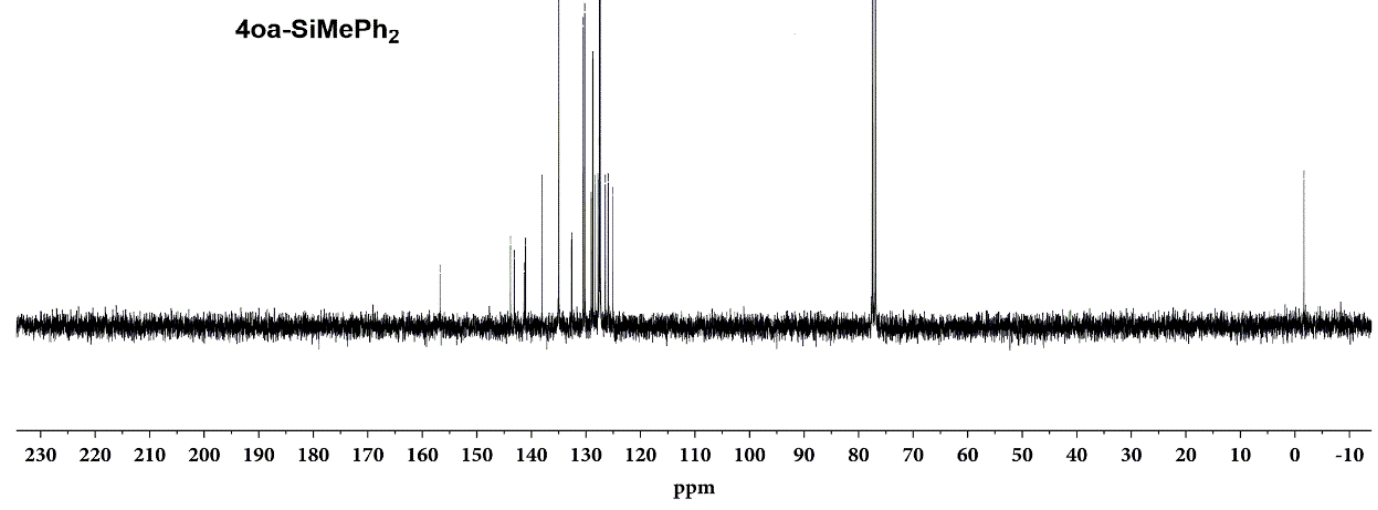

${ }^{1} \mathrm{H}$ NMR $(400 \mathrm{MHz})$ and ${ }^{13} \mathrm{C}\left\{{ }^{1} \mathrm{H}\right\}$ NMR (101 MHz) spectra of 4oa-SiMePh $2\left(\mathrm{rt}, \mathrm{CDCl}_{3}\right)$. 


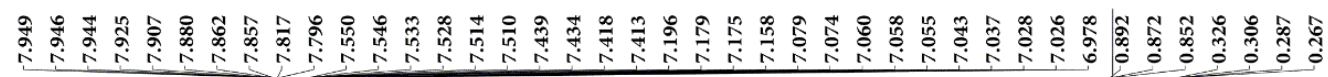<smiles>CCC(C)(C)C(=C(c1ccccc1)c1ccc2ccccc2c1)c1ccccc1</smiles>

4oa-SiEt ${ }_{3}$

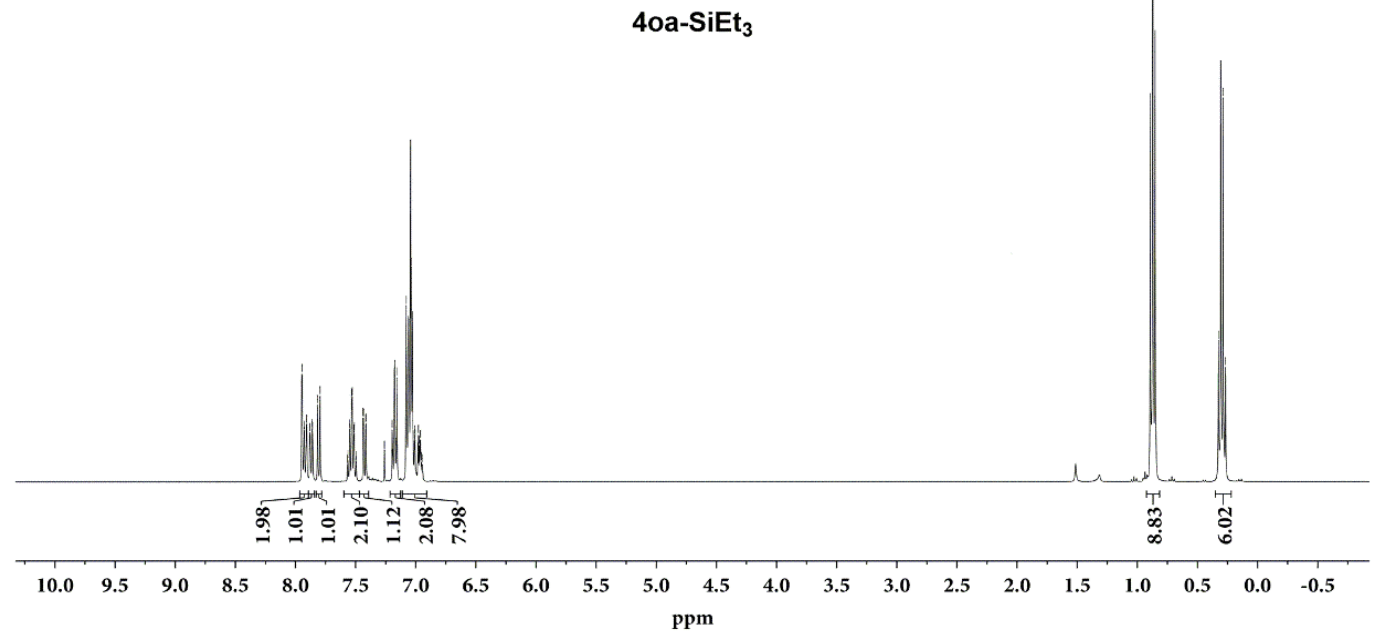

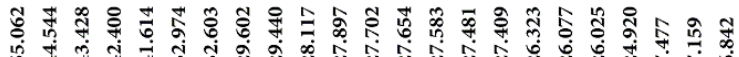

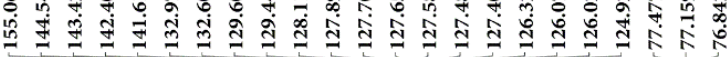

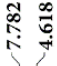

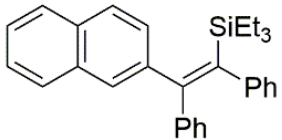

4oa-SiEt ${ }_{3}$

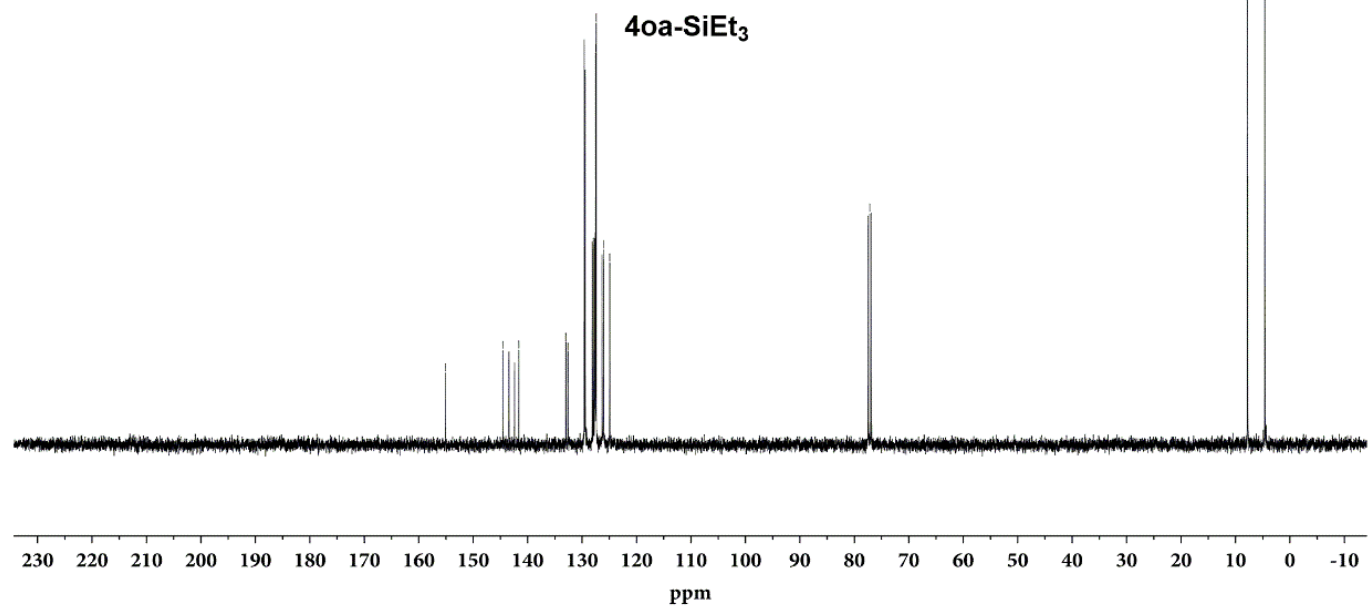

${ }^{1} \mathrm{H}$ NMR $(400 \mathrm{MHz})$ and ${ }^{13} \mathrm{C}\left\{{ }^{1} \mathrm{H}\right\}$ NMR $(101 \mathrm{MHz})$ spectra of 4oa-SiEt $\mathbf{3}_{\mathbf{3}}\left(\mathrm{rt}, \mathrm{CDCl}_{3}\right)$. 

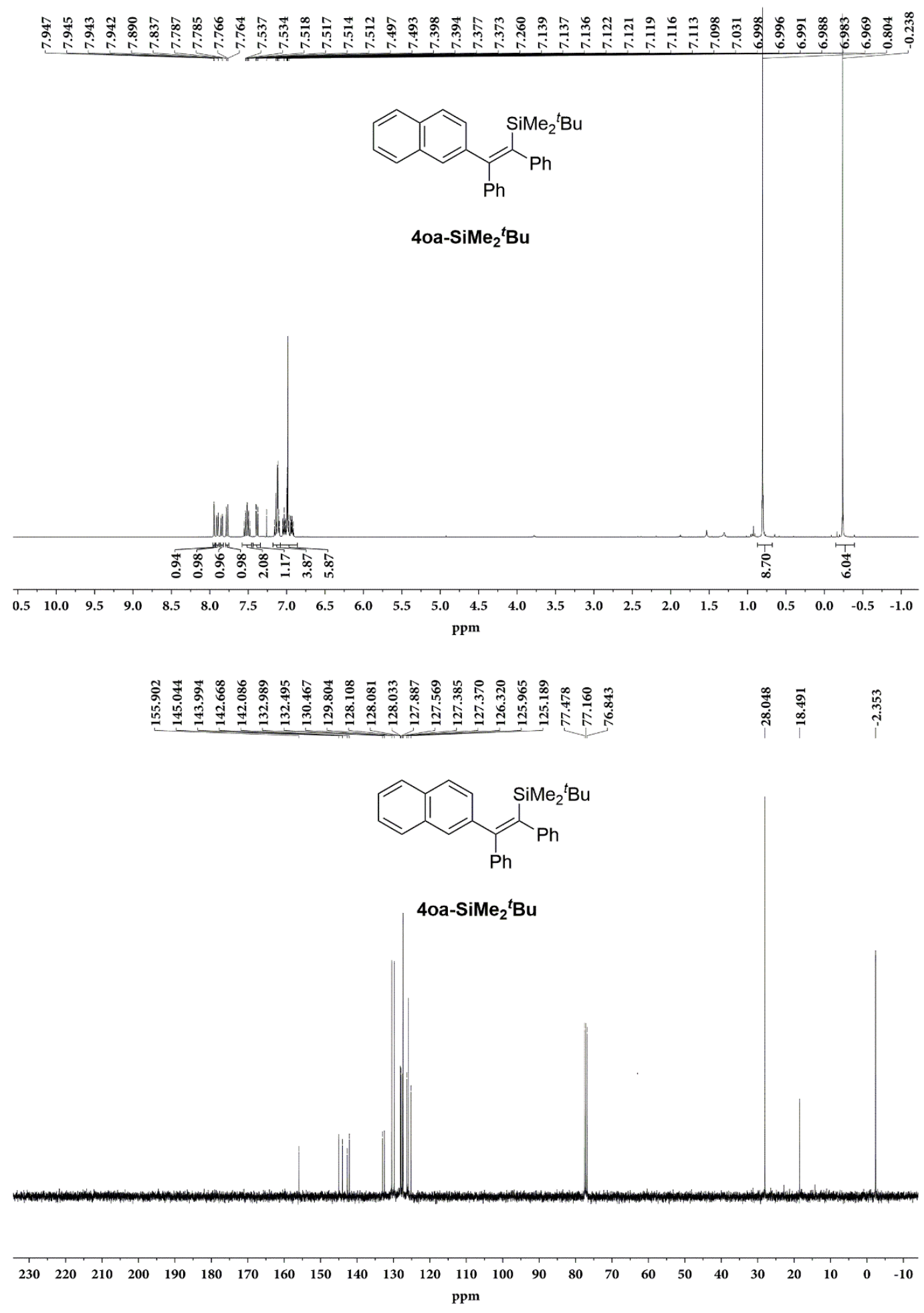

${ }^{1} \mathrm{H}$ NMR (400 MHz) and ${ }^{13} \mathrm{C}\left\{{ }^{1} \mathrm{H}\right\}$ NMR (101 MHz) spectra of 4oa-SiMe ${ }_{2}{ }^{t} \mathbf{B u}\left(\mathrm{rt}, \mathrm{CDCl}_{3}\right)$. 


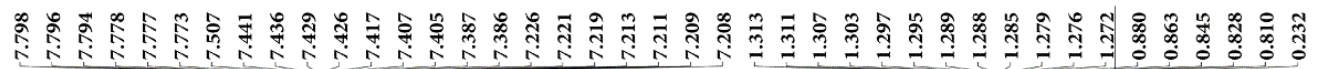

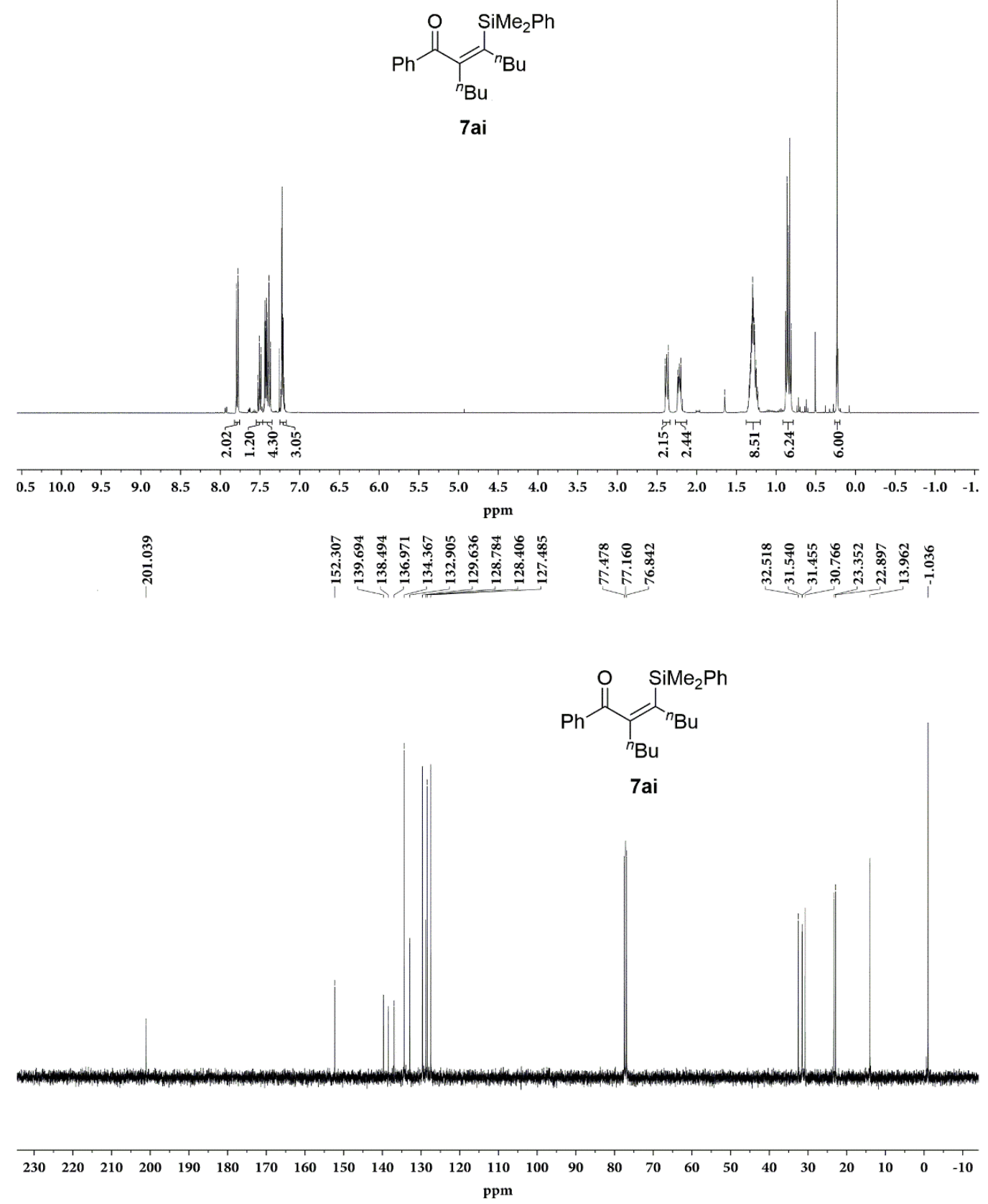

${ }^{1} \mathrm{H}$ NMR $(400 \mathrm{MHz})$ and ${ }^{13} \mathrm{C}\left\{{ }^{1} \mathrm{H}\right\}$ NMR (101 MHz) spectra of 7ai (rt, $\left.\mathrm{CDCl}_{3}\right)$. 


\section{References}

(1) (a) Okuda, Y.; Xu, J.; Ishida, T.; Wang, C.; Nishihara, Y. Nickel-Catalyzed Decarbonylative Alkylation of Aroyl Fluorides Assisted by Lewis-Acidic Organoboranes. ACS Omega 2018, 3, 13129-13140. (b) Wang, Z.; Wang, X.; Nishihara, Y. Nickel-catalysed decarbonylative borylation of aroyl fluorides. Chem. Commun. 2018, 54, 13969-13972. (c) Wang, X.; Wang, Z.; Liu, L.; Asanuma, Y.; Nishihara, Y. Nickel-Catalyzed Decarbonylative Stannylation of Acyl Fluorides under Ligand-Free Conditions. Molecules 2019, 24, 1671-1682. (d) Wang, X.; Wang, Z.; Asanuma, Y.; Nishihara, Y. Synthesis of 2-Substituted Propenes by Bidentate Phosphine-Assisted Methylenation of Acyl Fluorides and Acyl Chlorides with AlMe 3 . Org. Lett. 2019, 21, 3640-3643. (e) Wang, Z.; Wang, X.; Nishihara, Y. $\mathrm{PPh}_{3}$-Assisted Esterification of Acyl Fluorides with Ethers via $\mathrm{C}\left(s p^{3}\right)-\mathrm{O}$ Bond Cleavage Accelerated by TBAT. Catalysts 2019, 9, 574-586. (f) Kayumov, M.; Zhao, J.-N.; Mirzaakhmedov, S.; Wang, D.-Y.; Zhang, A. Synthesis of Arylstannanes via PalladiumCatalyzed Decarbonylative Coupling of Aroyl Fluorides. Adv. Synth. Catal. 2019, 362, 776-781. (g) Munoz, S. B.; Dang, H.; Ispizua-Rodriguez, X.; Mathew, T.; Prakash, G. K. S. Direct Access to Acyl Fluorides from Carboxylic Acids Using a Phosphine/Fluoride Deoxyfluorination Reagent System. Org. Lett. 2019, 21, 1659-1663.

(2) (a) Cirriez, V.; Rasson, C.; Hermant, T.; Petrignet, J.; Alvarez, J. D.; Robeyns, K.; Riant, O.; CopperCatalyzed Addition of Nucleophilic Silicon to Aldehydes. Angew. Chem., Int. Ed., 2013, 52, 1785-1788. (b) Guo, L.; Chatupheeraphat, A.; Rueping, M.; Decarbonylative Silylation of Esters by Combined Nickel and Copper Catalysis for the Synthesis of Arylsilanes and Heteroarylsilanes. Angew. Chem., Int. Ed., 2016, 55, 11810-11813. (c) Shishido, R.; Uesugi, M.; Takahashi, R.; Mita, T.; Ishiyama, T.; Kubota, K.; Ito, H. General Synthesis of Trialkyl- and Dialkylarylsilylboranes: Versatile Silicon Nucleophiles in Organic Synthesis. J. Am. Chem. Soc. 2020, 142, 14125-14133.

(3) (a) Park, K.; Bae, G.; Moon, J.; Choe, J.; Song, K. H.; Lee, S. Synthesis of Symmetrical and Unsymmetrical Diarylalkynes from Propiolic Acid Using Palladium-Catalyzed Decarboxylative Coupling. J. Org. Chem. 2010, 75, 6244-6251. (b) Pennell, M. N.; Turner, P. G.; Sheppard, T. D. Gold- and Silver-Catalyzed Reactions of Propargylic Alcohols in the Presence of Protic Additives. Chem. Eur. J. 2012, 18, 4748-4758.

(4) Fujimoto, T.; Ritter, T. PhenoFluorMix: Practical Chemoselective Deoxyfluorination of Phenols. Org. Lett. 2015, 17, 544-547.

(5) Jurkauskas, V.; Sadighi, J. P.; Buchwald, S. L. Conjugate Reduction of $\alpha, \beta$-Unsaturated Carbonyl Compounds Catalyzed by a Copper Carbene Complex. Org. Lett. 2003, 5, 2417-2420. 
(6) Munro-Leighton, C.; Blue, E. D.; Gunnoe, T. B. Anti-Markovnikov N-H and O-H Additions to ElectronDeficient Olefins Catalyzed by Well-Defined $\mathrm{Cu}(\mathrm{I})$ Anilido, Ethoxide, and Phenoxide Systems. J. Am. Chem. Soc. 2006, 128, 1446-1447.

(7) Shintani, R.; Kurata, H.; Nozaki, K. Intermolecular Three-Component Arylsilylation of Alkynes under Palladium/Copper Cooperative Catalysis. J. Org. Chem. 2016, 81, 3065-3069.

(8) Suginome, M.; Matsuda, T.; Nakamura, H.; Ito, Y. Regio- and stereoselective synthesis of (Z)- $\beta$ silylalkenylboranes by silaboration of alkynes catalyzed by palladium and platinum complexes. Tetrahedron $1999,55,8787-8800$. 